Florida International University FIU Digital Commons

FIU Electronic Theses and Dissertations

University Graduate School

3-28-2018

\title{
On the Performance of some Poisson Ridge Regression Estimators
}

Cynthia Zaldivar

Florida International University, czald001@fiu.edu

DOI: 10.25148 /etd.FIDC006538

Follow this and additional works at: https:// digitalcommons.fiu.edu/etd

Part of the Applied Statistics Commons, Multivariate Analysis Commons, Other Statistics and Probability Commons, Statistical Methodology Commons, $\underline{\text { Statistical Models Commons, }}$ Statistical Theory Commons, and the Theory and Algorithms Commons

\section{Recommended Citation}

Zaldivar, Cynthia, "On the Performance of some Poisson Ridge Regression Estimators" (2018). FIU Electronic Theses and Dissertations. 3669.

https://digitalcommons.fiu.edu/etd/3669

This work is brought to you for free and open access by the University Graduate School at FIU Digital Commons. It has been accepted for inclusion in FIU Electronic Theses and Dissertations by an authorized administrator of FIU Digital Commons. For more information, please contact dcc@fiu.edu. 


\title{
FLORIDA INTERNATIONAL UNIVERSITY
}

Miami, Florida

\section{ON THE PERFORMANCE OF SOME POISSON RIDGE REGRESSION} ESTIMATORS

\author{
A thesis submitted in partial fulfillment of the \\ requirements for the degree of \\ MASTER OF SCIENCE \\ in \\ STATISTICS \\ by \\ Cynthia Zaldivar
}




\section{To: Dean Michael R. Heithaus}

College of Arts, Sciences and Education

This thesis, written by Cynthia Zaldivar, and entitled On the Performance of Some Poisson Ridge Regression Estimators, having been approved in respect to style and intellectual content, is referred to you for judgment.

We have read this thesis and recommend that it be approved.

$\begin{array}{r}\text { Wensong Wu } \\ \hline \text { Florence George } \\ \hline \text { B. M. Golam Kibria, Major Professor }\end{array}$

Date of Defense: March 28, 2018

The thesis of Cynthia Zaldivar is approved.

Dean Michael R. Heithaus College of Arts, Sciences and Education

Andrés G. Gil

Vice President for Research and Economic Development and Dean of the University Graduate School

Florida International University, 2018 


\section{ACKNOWLEDGEMENTS}

I would like to thank the members of my committee - Dr. Florence George and Dr.

Wensong $\mathrm{Wu}$ - for their support and advice regarding this thesis. I would also like to thank Kristofer Månsson of Jönköping University for his help with the Monte Carlo simulation, finding data for the applications section, and answering any questions I had. Finally, I would like to thank my major professor, Dr. B. M. Golam Kibria, for his constant support throughout the process. His expertise on the subject matter has been crucial to the research. 


\begin{abstract}
OF THE THESIS
On the Performance of Some Poisson Ridge Regression Estimators

by

Cynthia Zaldivar
\end{abstract}

Florida International University, 2018

Miami, Florida

\title{
Professor B. M. Golam Kibria, Major Professor
}

Multiple regression models play an important role in analyzing and making predictions about data. Prediction accuracy becomes lower when two or more explanatory variables in the model are highly correlated. One solution is to use ridge regression. The purpose of this thesis is to study the performance of available ridge regression estimators for Poisson regression models in the presence of moderately to highly correlated variables.

As performance criteria, we use mean square error (MSE), mean absolute percentage error (MAPE), and percentage of times the maximum likelihood (ML) estimator produces a higher MSE than the ridge regression estimator.

A Monte Carlo simulation study was conducted to compare performance of the estimators under three experimental conditions: correlation, sample size, and intercept. It is evident from simulation results that all ridge estimators performed better than the ML estimator. We proposed new estimators based on the results, which performed very well compared to the original estimators. Finally, the estimators are illustrated using data on recreational habits. 
1 INTRODUCTION 1

1.1 Literature Review . . . . . . . . . . . . . . . . . . . . 1

1.2 Objective of the Thesis $\ldots \ldots \ldots \ldots \ldots$

2 METHODOLOGY 4

2.1 Poisson Ridge Regression . . . . . . . . . . . . . . . . . . . . . . . 4

2.2 Estimating the $\mathrm{k}$ Parameter $\ldots \ldots \ldots \ldots \ldots$

2.3 k Estimators Used . . . . . . . . . . . . . . . . . . . . . . . . . . . . . . 7

2.4 Criteria for Good Estimators $\ldots \ldots \ldots \ldots$

2.5 Inducing Outliers $\ldots \ldots \ldots \ldots \ldots \ldots \ldots \ldots \ldots \ldots$

3 THE MONTE CARLO SIMULATION 15

3.1 Simulation Technique . . . . . . . . . . . . . . . . 15

3.2 Simulation Results . . . . . . . . . . . . . . . . . . . 16

3.3 Performance as a Function of $\beta_{0} \ldots \ldots \ldots \ldots \ldots \ldots \ldots$

3.4 Performance as a Function of $\rho \ldots \ldots \ldots \ldots \ldots \ldots \ldots$

3.5 Performance as a Function of $n \ldots \ldots \ldots \ldots \ldots$

3.6 Best Performing Estimators . . . . . . . . . . . . . . . . . . . . 20

3.7 Proposed New Estimators . . . . . . . . . . . . . . . . . . . . . . . 21

3.8 Performance of New Estimators $\ldots \ldots \ldots \ldots$

4 APPLICATION 25

5 SUMMARY AND CONCLUDING REMARKS 28

$\begin{array}{ll}\text { REFERENCES } & 30\end{array}$

$\begin{array}{ll}\text { APPENDIX } & 33\end{array}$ 


\section{LIST OF TABLES}

TABLE

PAGE

3.1 Poisson Regression Simulation Results, $\mathrm{P}=4, \mathrm{n}=35$, Correlation $(\rho)=.85 \ldots \ldots$. . . 17

3.2 Poisson Regression Simulation Results, $\mathrm{P}=4, \mathrm{n}=600, \rho=.85 \ldots \ldots \ldots$

3.3 MSE Ratios (10\% Outliers), $\mathrm{P}=4, \mathrm{n}=35 \ldots \ldots \ldots \ldots$

3.4 Poisson Regression Simulation Results (New Estimators), $\mathrm{P}=4, \mathrm{n}=35, \rho=.85$. . . 22

3.5 Poisson Regression Simulation Results (New Estimators), $\mathrm{P}=4, \mathrm{n}=600$,

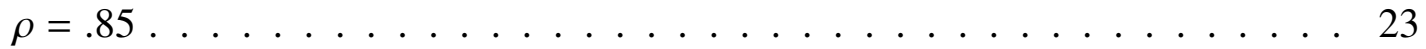

4.1 VIF for each regressor . . . . . . . . . . . . . . 26

4.2 MSE for Each k Estimator - Recreation Data . . . . . . . . . . . . . . . . 26

4.3 Significance of Coefficients from MLE and Selected New Estimators . . . . . . . . . 26

4.4 Estimated Regression Coefficients for Each Model . . . . . . . . . . . . . . . . . 27

A1 Poisson Regression Simulation Results, $\mathrm{P}=4, \mathrm{n}=35, \rho=.90 \quad \ldots \ldots$

A2 Poisson Regression Simulation Results, $\mathrm{P}=4, \mathrm{n}=35, \rho=.95 \ldots \ldots$

A3 Poisson Regression Simulation Results, $\mathrm{P}=4, \mathrm{n}=35, \rho=.99$. . . . . . . . 37

A4 Poisson Regression Simulation Results, $\mathrm{P}=4, \mathrm{n}=600, \rho=.90 \ldots \ldots$. . . . . . 38

A5 Poisson Regression Simulation Results, $\mathrm{P}=4, \mathrm{n}=600, \rho=.95 \ldots \ldots$

A6 Poisson Regression Simulation Results, $\mathrm{P}=4, \mathrm{n}=600, \rho=.99$. . . . . . . . 40

A7 Poisson Regression Simulation Results (New Estimators), $\mathrm{P}=4, \mathrm{n}=35, \rho=.9041$

A8 Poisson Regression Simulation Results (New Estimators), $\mathrm{P}=4, \mathrm{n}=35, \rho=.9542$

A9 Poisson Regression Simulation Results (New Estimators), $\mathrm{P}=4, \mathrm{n}=35, \rho=.9943$

A10 Poisson Regression Simulation Results (New Estimators), $\mathrm{P}=4, \mathrm{n}=600$, $\rho=.90 \ldots \ldots \ldots \ldots \ldots \ldots \ldots \ldots$

A11 Poisson Regression Simulation Results (New Estimators), $\mathrm{P}=4, \mathrm{n}=600$,

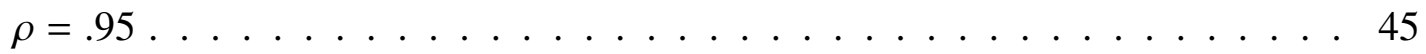

A12 Poisson Regression Simulation Results (New Estimators), $\mathrm{P}=4, \mathrm{n}=600$,

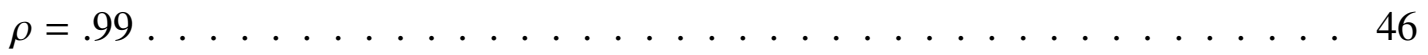




\section{LIST OF FIGURES}

FIGURE

PAGE

$3.1 \quad$ MSE for each k estimator, $n=35, \rho=.85 \ldots \ldots \ldots \ldots \ldots \ldots$

3.2 Estimators with Smallest MSE $\left(\mathrm{n}=35, \rho=.85, \beta_{0}=-1\right) \ldots \ldots \ldots 24$

A1 MSE for each k estimator, $\rho=.90 \ldots \ldots \ldots \ldots \ldots \ldots$

A2 MSE for each k estimator, $\rho=.95 \ldots \ldots \ldots \ldots \ldots \ldots \ldots$

A3 MSE for each k estimator, $\rho=.99 \ldots \ldots \ldots \ldots \ldots$ 


\section{CHAPTER 1}

\section{INTRODUCTION}

\subsection{Literature Review}

When any pair of independent variables are highly correlated, the corresponding regression coefficients will have large standard errors, which means that the regression coefficients are unstable. When two independent variables are highly correlated, they are not orthogonal, that is the regressors have a near linear relation. In such a situation, the coefficients are less likely to be statistically significant. Inferences made using such a model will not be correct. This type of situation is referred to as multicollinearity. (Allen, 1997)

Two methods of dealing with multicollinearity are collecting more data and model respecification. While collecting more data may be the best way to correct multicollinearity, it is not always possible to collect additional data. There are often financial constraints which render the solution impossible. Finances aside, the process, individuals, or items being studied may no longer be accessible for sampling. Collecting more data may not solve the issue of multicollinearity if the problem arises from limitations of the model or population. If multicollinearity is caused by some characteristic of the model, such as the presence of two highly correlated variables, model respecification may help alleviate the issue. The discovery and implementation of a simple function, such as $x=x 1 / x 2$ or $x=x 1 x 2$ may aid the issue while preserving the information gained from the regressors. One effective method of dealing with multicollinearity is elimination of any highly correlated variables. While this kind of brute force method may totally remove the issue, the information gained from any removed regressors will be lost. The loss of information can be an unacceptable consequence to many researchers. (Montgomery, 2012) 
The widely used method of dealing with multicollinearity is through the use of ridge regression, in which regression coefficients are calculated as such:

$$
\hat{\beta}_{R}(k)=\left(X^{\prime} X+k I\right)^{-1} X^{\prime} y .
$$

Here $\mathrm{X}$ is an $n x(p+1)$ matrix, $\mathrm{p}$ being the number of independent variables used in the model.For values $k \geq 0$ the mean square error (MSE) is smaller than that of the least squares estimator:

$$
\hat{\beta}=\left(X^{\prime} X\right)^{-1} X^{\prime} y
$$

The ridge regression method of dealing with multicollinearity was originally proposed by Hoerl and Kennard (1970). Many researchers have proposed estimates for k, including Lawless and Wang (1976), Kibria (2003), Alkhamisi and Shukur (2008), Kibria and Muñiz (2009), Kibria and Banik (2016), and Asar and Genç (2017), among others.

There has been limited research on ridge regression using non-Gaussian regression models, such as the Poisson regression model. Månsson and Shukur (2011) evaluated the performance of several k estimators for Poisson ridge regression using Monte Carlo simulations. The present thesis expands on that study by evaluating more $\mathrm{k}$ estimators and proposing some new k estimators for Poisson ridge regression models.

The Poisson regression model has a large amount of applications, especially in economics. A Poisson model is useful when the response variable includes countable, independent events, such as the total number of car accidents per week on a specific highway, the number of calls received per minute at a call center, or number of credit defaults per year (Greene, 2012). Further applications will be discussed in Section 4 of this thesis. 


\subsection{Objective of the Thesis}

The problem of estimating $\mathrm{k}$ is the subject of many research papers and has not been solved yet. The different methods proposed in research each have advantages and disadvantages. It is necessary to compare different estimators under different error distributions under the same conditions. The purpose of this research is to compare popular ridge parameter estimators using the following criteria:

1. Smallest mean squared error (MSE)

2. Mean absolute percentage error (MAPE)

3. Performance of ridge regression estimator compared to the least squares estimator, in terms of the percentage of times in simulation that the ridge regression estimator produces a lower MSE than the least squares estimator.

The present research extends Poisson Ridge Regression (PRR) research by Månsson and Shukur (2011). Ridge regression estimators for Poisson regression models will be compared to the maximum likelihood estimation (MLE) method using Monte Carlo simulations.

The organization of the thesis is as follows. We define different types of ridge regression estimators of $\mathrm{k}$ in Chapter 2. A Monte Carlo simulation study has been conducted in Chapter 3. In Chapter 4, the empirical application of the Poisson ridge regression is presented. The summary and concluding remarks are given in Chapter 5. 


\section{CHAPTER 2}

\section{METHODOLOGY}

\subsection{Poisson Ridge Regression}

In Poisson regression, it is standard to cross-section data in applications of $\mathrm{n}$ independent observations. The $i^{\text {th }}$ observation is $\left(y_{i}, \mathbf{x}_{i}\right)$. Here $y_{i}$, the dependent variable, represents the number of occurrences of the event of interest and $\mathbf{x}_{i}^{\prime}=\left\{x_{1 i}, x_{2 i}, \ldots, x_{p i}\right\}$ is a vector of $\mathrm{p}$ linearly independent regressors that are thought to determine $y_{i} \cdot y_{i}$ given $x_{i}$ is Poisson distributed, with the density function:

$$
f\left(y_{i} \mid x_{i}\right)=\frac{e^{-\mu_{i}} \mu_{i}^{y_{i}}}{y_{i} !}, y_{i}=0,1,2, \ldots
$$

The mean parameter, which represents the average rate of occurrence of the event of interest, is calculated as:

$$
\mu_{i}=e^{x_{i}^{\prime} \beta}
$$

where $\beta$ is a vector of parameters from the regression model.

The Maximum Likelihood Estimator (MLE) for $\beta$ is found using the iterative weighted least square (IWLS) algorithm:

$$
\begin{aligned}
& \hat{\beta}_{M L E}=\left(X^{\prime} \hat{W} X\right)^{-1} X^{\prime} \hat{W} \hat{z}, \\
& \text { where } \hat{W}=\operatorname{diag}\left(\hat{\mu}_{i}\right), \quad \hat{\mu}_{i}=e^{x_{i} \hat{\beta}}, \quad \text { and } \hat{z}=\log \left(\hat{\mu}_{i}\right)+\frac{y_{i}-\hat{\mu}_{i}}{\hat{\mu}_{i}} .
\end{aligned}
$$

The Poisson ridge regression estimator is calculated as:

$$
\hat{\beta}_{R}(k)=\left(X^{\prime} \hat{W} X+k I\right)^{-1} X^{\prime} \hat{W} \hat{z}=\left(X^{\prime} \hat{W} X+k I\right)^{-1} X^{\prime} \hat{W} X \hat{\beta}_{M L E}=U \hat{\beta}_{M L E} .
$$


where $U=\left(X^{\prime} \hat{W} X+k I\right)^{-1} X^{\prime} \hat{W} X$

The MSE of $\hat{\beta}_{R}(k)$ can be calculated as follows:

$$
\begin{aligned}
M S E\left(\hat{\beta}_{R}(k)\right)= & M S E\left(U \hat{\beta}_{M L E}\right)=E\left(U \hat{\beta}_{M L E}-\beta\right)^{\prime}\left(U \hat{\beta}_{M L E}-\beta\right) \\
= & E\left(\hat{\beta}_{M L E}^{\prime} U^{\prime} U \hat{\beta}_{M L E}\right)-E\left(\beta^{\prime} U \hat{\beta}_{M L E}\right)-E\left(\hat{\beta}_{M L E}^{\prime} U^{\prime} \beta\right)+E\left(\beta^{\prime} \beta\right) \\
= & E\left(\hat{\beta}_{M L E}^{\prime} U^{\prime} U \hat{\beta}_{M L E}\right)-E\left(\beta^{\prime} U^{\prime} U \hat{\beta}_{M L E}\right)-E\left(\hat{\beta}_{M L E}^{\prime} U^{\prime} U \beta\right)+E\left(\hat{\beta}_{M L E}^{\prime} U^{\prime} U \beta\right) \\
& +E\left(\beta^{\prime} U^{\prime} U \beta\right)-E\left(\beta^{\prime} U^{\prime} U \beta\right)+E\left(\beta^{\prime} U^{\prime} U \hat{\beta}_{M L E}\right) \\
& -E\left(\beta^{\prime} U \hat{\beta}_{M L E}\right)-E\left(\hat{\beta}_{M L E}^{\prime} U^{\prime} \beta\right)+E\left(\beta^{\prime} \beta\right)
\end{aligned}
$$

Lee and Silvapulle (1988) found that, for large values of $n$, the distribution of $\hat{\beta}_{M L E}$ is $\hat{\beta}_{M L E} \sim N\left(\beta,\left(X^{\prime} \hat{W} X\right)^{-1}\right)$. Then:

$$
\begin{aligned}
M S E\left(\hat{\beta}_{R}(k)\right)= & E\left[\left(\hat{\beta}_{M L E}-\beta\right)^{\prime} U^{\prime} U\left(\hat{\beta}_{M L E}-\beta\right)\right] \\
& -\beta^{\prime} U^{\prime} U \beta+\beta^{\prime} U^{\prime} U \beta+\beta^{\prime} U^{\prime} U \beta-\beta^{\prime} U \beta-\beta^{\prime} U^{\prime} \beta+\beta^{\prime} \beta \\
= & E\left[\left(\hat{\beta}_{M L E}-\beta\right)^{\prime} U^{\prime} U\left(\hat{\beta}_{M L E}-\beta\right)\right]+\beta^{\prime}\left(U-I_{p}\right)^{\prime}\left(U-I_{p}\right) \beta \\
= & E\left[\operatorname{tr}\left[\left(\hat{\beta}_{M L E}-\beta\right)^{\prime} U^{\prime} U\left(\hat{\beta}_{M L E}-\beta\right)\right]\right]+\beta^{\prime}\left(U-I_{p}\right)^{\prime}\left(U-I_{p}\right) \beta \\
= & E\left[\operatorname{tr}\left[U\left(\hat{\beta}_{M L E}-\beta\right)\left(\hat{\beta}_{M L E}-\beta\right)^{\prime} U^{\prime}\right]\right]+\beta^{\prime}\left(U-I_{p}\right)^{\prime}\left(U-I_{p}\right) \beta \\
= & \operatorname{tr}\left[U E\left[\left(\hat{\beta}_{M L E}-\beta\right)\left(\hat{\beta}_{M L E}-\beta\right)^{\prime}\right] U^{\prime}\right]+\beta^{\prime}\left(U-I_{p}\right)^{\prime}\left(U-I_{p}\right) \beta \\
= & \operatorname{tr}\left[U\left(X^{\prime} \hat{W} X\right)^{-1} U^{\prime}\right]+\beta^{\prime}\left(U-I_{p}\right)^{\prime}\left(U-I_{p}\right) \beta \\
= & \left.\operatorname{tr}\left[X^{\prime} \hat{W} X+k I_{p}\right)^{-2} X^{\prime} \hat{W} X\right] \\
& +\beta^{\prime}\left(-k\left(X^{\prime} \hat{W} X+k I_{p}\right)^{-1}\right)^{\prime}\left(-k\left(X^{\prime} \hat{W} X+k I_{p}\right)^{-1}\right) \beta \\
= & \operatorname{tr}\left(\left(X^{\prime} \hat{W} X+k I_{p}\right)^{-2} X^{\prime} \hat{W} X\right)+\beta^{\prime}\left(k^{2}\left(X^{\prime} \hat{W} X+k I_{p}\right)^{-2}\right) \beta
\end{aligned}
$$


Let $C=X^{\prime} \hat{W} X$ and $\Lambda=\operatorname{diag}\left(\lambda_{1}, \lambda_{2}, \ldots, \lambda_{p}\right)$ be the eigenvalues of $C$. Suppose there exists an orthogonal matrix $\mathrm{D}$ such that $D^{\prime} C D=\Lambda$, then:

$$
\begin{aligned}
= & \operatorname{tr}\left(\left(X^{\prime} \hat{W} X+k I_{p}\right)^{-2} X^{\prime} \hat{W} X\right)+\beta^{\prime}\left(k^{2}\left(X^{\prime} \hat{W} X+k I_{p}\right)^{-2}\right) \beta \\
= & \operatorname{tr}\left(D D^{\prime}\left(X^{\prime} \hat{W} X+k I_{p}\right)^{-1} D D^{\prime}\left(X^{\prime} \hat{W} X+k I_{p}\right)^{-1} D D^{\prime} X^{\prime} \hat{W} X\right) \\
& +k^{2}(D \beta)^{\prime}\left(D^{\prime} X^{\prime} \hat{W} X D+k D D^{\prime}\right)^{-2}(D \beta) \\
= & \operatorname{tr}\left(D^{\prime}\left(X^{\prime} \hat{W} X+k I_{p}\right)^{-1} D D^{\prime}\left(X^{\prime} \hat{W} X+k I_{p}\right)^{-1} D D^{\prime} X^{\prime} \hat{W} X D\right)+k^{2} \sum_{i=1}^{p} \frac{\alpha_{i}^{2}}{\left(\lambda_{i}+k\right)^{2}} \\
= & \operatorname{tr}\left(\left(D^{\prime} X^{\prime} \hat{W} X D+k I_{p}\right)^{-1}\left(D^{\prime} X^{\prime} \hat{W} X D+k I_{p}\right)^{-1} D^{\prime} X^{\prime} \hat{W} X D\right)+k^{2} \sum_{i=1}^{p} \frac{\alpha_{i}^{2}}{\left(\lambda_{i}+k\right)^{2}} \\
= & \operatorname{tr}\left(\left(\Lambda+k I_{p}\right)^{-1}\left(\Lambda+k I_{p}\right)^{-1} \Lambda\right)+k^{2} \sum_{i=1}^{p} \frac{\alpha_{i}^{2}}{\left(\lambda_{i}+k\right)^{2}} \\
= & \sum_{i=1}^{p} \frac{\lambda_{i}}{\left(\lambda_{i}+k\right)^{2}}+k^{2} \sum_{i=1}^{p} \frac{\alpha_{i}^{2}}{\left(\lambda_{i}+k\right)^{2}}=\operatorname{Var}\left(\hat{\beta}^{R}(k)\right)+\left(\operatorname{Bias}^{2}\left(\hat{\beta}^{R}(k)\right)\right)^{2}
\end{aligned}
$$

where $\lambda_{i}$ is the $i^{\text {th }}$ eigenvalue of $\mathrm{C}$ and $\alpha=D \beta$

Månsson (2011)

\subsection{Estimating the $\mathrm{k}$ Parameter}

Let $\hat{\alpha}(k)=\left(X^{*^{\prime}} X^{*}+k I\right)^{-1} X^{*^{\prime}} y$,

where $k=\operatorname{diag}\left(k_{1}, k_{1}, \ldots, k_{p}\right), k_{i}>0$,

$\operatorname{MSE}(\hat{\alpha}(\hat{k}))=\sum_{i=1}^{p} \frac{\lambda_{i}}{\left(\lambda_{i}+\hat{k}_{i}\right)^{2}}+\sum_{i=1}^{p} \frac{\hat{k}_{i}^{2} \hat{\alpha}_{i}^{2}}{\left(\lambda_{i}+\hat{k}_{i}\right)^{2}}$

The value of which $k_{i}$ minimizes $M S E(\hat{\alpha}(\hat{k}))$ is:

$$
\hat{k}_{i}=\frac{1}{\hat{\alpha}_{i}^{2}}
$$

Hoerl and Kennard (1970) 


\section{$2.3 \mathrm{k}$ Estimators Used}

This thesis will analyze the performance of 50 different $\mathrm{k}$ estimators. The $\mathrm{k}$ estimators used in this thesis are summarized below.

The first two k are from Hoerl and Kennard (1970):

$$
\begin{aligned}
& H K=\hat{k}_{H K}=\frac{1}{\hat{\alpha}_{\text {max }}^{2}} \\
& H K_{2}=\hat{K}_{H K 2}=\frac{1}{\sum_{i=1}^{p} \hat{\alpha}_{i}^{2}}
\end{aligned}
$$

A different estimator, produced by taking the harmonic mean of $\hat{k}_{i}$ was suggested by Hoerl et al. (1975):

$$
H K_{B}=\hat{k}_{H K B}=\frac{p}{\sum_{i=1}^{p} \hat{\alpha}_{i}^{2}}=\frac{p}{\hat{\alpha}^{\prime} \hat{\alpha}}
$$

The next k estimator was proposed by Lawless and Wang (1976):

$$
\hat{k}_{L W i}=\frac{1}{\lambda_{i} \hat{\alpha}_{i}^{2}}
$$

and suggest taking the harmonic mean of (2.13):

$$
L W=\hat{k}_{L W}=\frac{p}{\sum_{i=1}^{p} \lambda_{i} \hat{\alpha}_{i}^{2}}=\frac{p}{\hat{\alpha}^{\prime} X^{\prime} X \hat{\alpha}}
$$

The next k estimator was proposed by Hocking et al. (1976): 


$$
H S L=\hat{k}_{H S L}=\frac{\sum_{i=1}^{p}\left(\lambda_{i} \hat{\alpha}_{i}\right)^{2}}{\left(\sum_{i=1}^{p} \lambda_{i} \hat{\alpha}_{i}^{2}\right)^{2}}
$$

The next three k estimators, proposed by Kibria (2003), take the arithmetic mean, geometric mean, and median of the ridge estimator $\hat{k}_{i}$ :

$$
\begin{aligned}
& A M=\hat{k}_{A M}=\frac{1}{p} \sum_{i=1}^{p} \frac{1}{\hat{\alpha}_{i}^{2}} \\
& G M=\hat{k}_{G M}=\frac{1}{\left(\prod_{i=1}^{p} \hat{\alpha}_{i}^{2}\right)^{1 / p}} \\
& \text { Med }=\hat{k}_{\text {Med }}=\operatorname{Median}\left(\frac{1}{\hat{\alpha}_{i}^{2}}\right)
\end{aligned}
$$

Khalaf and Shukur (2005) suggested the following k estimator:

$$
K S=\hat{K}_{K S}=\frac{\lambda_{\max }}{(n-p)+\lambda_{\max } \hat{\alpha}_{\max }^{2}}
$$

Alkhamisi et al. (2006) proposed the following:

$$
\begin{aligned}
& K S_{A}=\hat{K}_{\text {Arith }}^{K S}=\frac{1}{p} \sum_{i=1}^{p}\left(\frac{\lambda_{i}}{(n-p)+\lambda_{i} \hat{\alpha}_{i}^{2}}\right) \\
& K S_{\text {Max }}=\hat{K}_{\text {Max }}^{K S}=\operatorname{Max}\left\{\frac{\lambda_{i}}{(n-p)+\lambda_{i} \hat{\alpha}_{i}^{2}}\right\} \\
& K S_{\text {Med }}=\hat{K}_{\text {Med }}^{K S}=\text { Median }\left\{\frac{\lambda_{i}}{(n-p)+\lambda_{i} \hat{\alpha}_{i}^{2}}\right\}
\end{aligned}
$$

Muñiz and Kibria (2009) proposed some new estimators, the first being the geometric 
mean of $\left(\frac{\lambda_{i}}{(n-p)+\lambda_{i} \hat{\alpha}_{i}^{2}}\right)$

$$
K M_{1}=\hat{K}_{G M}^{K S}=\hat{K}_{K M 1}=\prod_{i=1}^{p}\left(\frac{\lambda_{i}}{(n-p)+\lambda_{i} \hat{\alpha}_{i}^{2}}\right)^{1 / p}
$$

They also proposed the following six estimators, based on square root transformations, as suggested by Alkhamisi and Shukur (2008):

$$
\begin{aligned}
& K M_{2}=\hat{K}_{K M 2}=\operatorname{Max}\left\{\sqrt{\hat{\alpha}_{i}^{2}}\right\} \\
& K M_{3}=\hat{K}_{K M 3}=\operatorname{Max}\left\{\frac{1}{\sqrt{\hat{\alpha}_{i}^{2}}}\right\} \\
& K M_{4}=\hat{K}_{K M 4}=\left(\prod_{i=1}^{p} \sqrt{\hat{\alpha}_{i}^{2}}\right)^{1 / p} \\
& K M_{5}=\hat{K}_{K M 5}=\left(\prod_{i=1}^{p} \sqrt{\frac{1}{\hat{\alpha}_{i}^{2}}}\right)^{1 / p} \\
& K M_{6}=\hat{K}_{K M 6}=\operatorname{Median}\left\{\sqrt{\hat{\alpha}_{i}^{2}}\right\} \\
& K M_{7}=\hat{K}_{K M 7}=\operatorname{Median}\left\{\frac{1}{\sqrt{\hat{\alpha}_{i}^{2}}}\right\}
\end{aligned}
$$

Muñiz et al. (2012) proposed the following estimators:

$$
\begin{aligned}
& K M_{8}=\hat{K}_{K M 8}=\operatorname{Max}\left\{\sqrt{\frac{(n-p)+\lambda_{\max } \hat{\alpha}_{i}^{2}}{\lambda_{\max }}}\right\} \\
& K M_{9}=\hat{K}_{K M 9}=\operatorname{Max}\left\{\sqrt{\frac{\lambda_{\max }}{(n-p)+\lambda_{\max } \hat{\alpha}_{i}^{2}}}\right\} \\
& K M_{10}=\hat{K}_{K M 10}=\prod_{i=1}^{p}\left(\sqrt{\frac{(n-p)+\lambda_{\max } \hat{\alpha}_{i}^{2}}{\lambda_{\max }}}\right)^{1 / p}
\end{aligned}
$$




$$
\begin{aligned}
& K M_{11}=\hat{K}_{K M 11}=\prod_{i=1}^{p}\left(\sqrt{\frac{\lambda_{\max }}{(n-p)+\lambda_{\max } \hat{\alpha}_{i}^{2}}}\right)^{1 / p} \\
& K M_{12}=\hat{K}_{K M 12}=\text { Median }\left\{\sqrt{\frac{(n-p)+\lambda_{\max } \hat{\alpha}_{i}^{2}}{\lambda_{\max }}}\right\}
\end{aligned}
$$

The next two estimators were proposed by Kibria et al. (2011):

$$
\begin{aligned}
& K M_{13}=\hat{K}_{K M 13}=\prod_{i=1}^{p}\left(\frac{(n-p)+\lambda_{\max } \hat{\alpha}_{i}^{2}}{\lambda_{\max }}\right)^{1 / p} \\
& K M_{14}=\hat{K}_{K M 14}=\prod_{i=1}^{p}\left(\frac{\lambda_{\max }}{(n-p)+\lambda_{\max } \hat{\alpha}_{i}^{2}}\right)^{1 / p}
\end{aligned}
$$

Khalaf (2012) proposed the following modification of $\hat{k}_{H K}$ :

$$
G K=\hat{K}_{G K}=\hat{K}_{H K}+\frac{2}{\left(\lambda_{\max }+\lambda_{\min }\right)^{\prime}}
$$

Nomura (1988) suggested the following:

$$
H M O=\hat{K}_{H M O}=\frac{p}{\sum_{i=1}^{p}\left(\hat{\alpha}_{i}^{2} /\left(1+\left(1+\lambda_{i} \sqrt{\hat{\alpha}_{i}^{2}}\right)\right)\right)}
$$

The following four estimators were proposed by Dorugade (2013):

$$
\begin{aligned}
& A D_{H M}=\hat{K}_{H M}^{A D}=\frac{2 p}{\sum_{i=1}^{p} \lambda_{\max } \hat{\alpha}_{i}^{2}} \\
& A D_{M e d}=\hat{K}_{M e d}^{A D}=\operatorname{Median}\left\{\frac{2}{\lambda_{\max } \hat{\alpha}_{i}^{2}}\right\} \\
& A D_{G M}=\hat{K}_{G M}^{A D}=\frac{2}{\lambda_{\max }\left(\prod_{i=1}^{p} \hat{\alpha}_{i}^{2}\right)^{1 / p}}
\end{aligned}
$$




$$
A D_{A M}=\hat{K}_{A M}^{A D}=\frac{2}{\lambda_{\max } p} \sum_{i=1}^{p} \frac{1}{\hat{\alpha}_{i}^{2}}
$$

Asar and Aşır (2017) recently proposed the following nine estimators:

$$
\begin{aligned}
& Y_{1}=\hat{K}_{Y 1}=\frac{1}{p} \sum_{i=1}^{p} \sqrt{\frac{1}{\lambda_{i} \hat{\alpha}_{i}^{2}}} \\
& Y_{2}=\hat{K}_{Y 2}=\left(\prod_{i=1}^{p} \sqrt{\frac{1}{\lambda_{i} \hat{\alpha}_{i}^{2}}}\right)^{1 / p} \\
& Y_{3}=\hat{K}_{Y 3}=\operatorname{Median}\left\{\sqrt{\frac{1}{\lambda_{i} \hat{\alpha}_{i}^{2}}}\right\} \\
& Y_{4}=\hat{K}_{Y 4}=\operatorname{Max}\left\{\sqrt{\frac{1}{\lambda_{i} \hat{\alpha}_{i}^{2}}}\right\} \\
& Y_{5}=\hat{K}_{Y 5}=\operatorname{Median}\left\{\sqrt{\lambda_{i} \hat{\alpha}_{i}^{2}}\right\} \\
& Y_{6}=\hat{K}_{Y 6}=\operatorname{Max}\left\{\sqrt{\lambda_{i} \hat{\alpha}_{i}^{2}}\right\} \\
& Y_{9}=\hat{K}_{Y 9}=\frac{p}{\sum_{i=1}^{p} \sqrt{\frac{1}{\lambda_{i} \hat{\alpha}_{i}^{2}}}} \\
& Y_{8}=\hat{K}_{Y 8}=\frac{1}{\sum_{i=1}^{p} \sqrt{\lambda_{i} \hat{\alpha}_{i}^{2}}} \sqrt{\lambda_{i} \hat{\alpha}_{i}^{2}} \\
& p \\
& Y_{i=1}
\end{aligned}
$$

Al-Hassan (2010) proposed the following estimator:

$$
A H=\hat{K}_{A H}=\frac{\lambda_{\max } \sum_{i=1}^{p}\left(\lambda_{i} \hat{\alpha}_{i}^{2}\right)+\left(\sum_{i=1}^{p} \lambda_{i} \hat{\alpha}_{i}^{2}\right)^{2}}{\lambda_{\max } \sum_{i=1}^{p} \lambda_{i} \hat{\alpha}_{i}^{2}}
$$


Batah and Gore (2009) suggested the following k estimator:

$$
F G=\hat{K}_{F G}=\frac{p}{\sum_{i=1}^{p}\left(\hat{\alpha}_{i}^{2} /\left(\left(\frac{\hat{\alpha}_{i}^{4} \lambda_{i}^{2}}{4}+6 \hat{\alpha}_{i}^{4} \lambda_{i}\right)^{1 / 2}-6 \hat{\alpha}_{i}^{2} \lambda_{i}\right)\right)}
$$

Dorugade (2014) proposed the following:

$$
\begin{aligned}
& A S=\hat{K}_{A S}=\frac{1}{\hat{\alpha}_{\text {max }}^{2}}+\frac{1}{\lambda_{\max }} \\
& A S_{\text {Max }}=\hat{K}_{\text {Max }}^{A S}=\operatorname{Max}\left\{\frac{1}{\hat{\alpha}_{i}^{2}}+\frac{1}{\lambda_{i}}\right\} \\
& A S_{\text {Min }}=\hat{K}_{\text {Min }}^{A S}=\frac{1}{\operatorname{Min}\left\{\frac{1}{\hat{\alpha}_{i}^{2}}+\frac{1}{\lambda_{i}}\right\}}
\end{aligned}
$$

Adnan et al. (2014) proposed the following estimators:

$$
\begin{aligned}
& N_{1}=\hat{K}_{H M}^{N 1}=\frac{\sqrt{5} p}{\lambda_{\max } \sum_{i=1}^{p} \hat{\alpha}_{i}^{2}} \\
& N_{2}=\hat{K}_{H M}^{N 2}=\frac{p}{\sqrt{\lambda_{\max } \sum_{i=1}^{p} \hat{\alpha}_{i}^{2}}} \\
& N_{3}=\hat{K}_{H M}^{N 3}=\frac{2 p}{\sum_{i=1}^{p}\left(\lambda_{i}^{1 / 4}\right) \sum_{i=1}^{p} \hat{\alpha}_{i}^{2}} \\
& N_{4}=\hat{K}_{H M}^{N 4}=\frac{2 p}{\sqrt{\sum_{i=1}^{p} \lambda_{i}} \sum_{i=1}^{p} \hat{\alpha}_{i}^{2}}
\end{aligned}
$$

The best performing of the $50 \mathrm{k}$ estimators will be used as the basis for new k estimators. The performance of those new estimators will then be assessed using the same criteria, outlined below. 


\subsection{Criteria for Good Estimators}

The following criteria will be used to judge the performance of the $50 \mathrm{k}$ estimators:

\section{Average MSE}

2. Percentage of times PRR outperforms MLE (performance)

3. Average mean absolute percentage error (MAPE).

Average MSE is calculated as follows:

$$
M S E_{A v}=\frac{\sum_{i=1}^{r} S E_{i}}{r}=\frac{\sum_{i=1}^{r}(\hat{\beta}-\beta)_{i}^{\prime}(\hat{\beta}-\beta)_{i}}{r}, \quad \text { where } r=\text { number of replicates. }
$$

The performance of PRR versus MLE is defined as the percentage of times, among the $r$ replicates, that PRR has a smaller MSE than that of MLE.

MAPE is calculated as follows:

$$
\begin{aligned}
& \operatorname{MAPE}_{A v}=\frac{1}{r} \sum_{i=1}^{r} \operatorname{MAPE}_{i}(\hat{\beta}), \\
& \text { where } \operatorname{MAPE}_{i}(\hat{\beta})=\frac{1}{p} \sum_{i=1}^{p} \frac{\left|(\beta-\hat{\beta})_{i}\right|}{\left|\beta_{i}\right|} .
\end{aligned}
$$

\subsection{Inducing Outliers}

Since the thesis uses simulated data, it is important to test how the models perform with outliers, since real-world data sometimes contains outliers. Ten percent of the points in the response variable, $\mathrm{y}$, were randomly selected and set to $y_{i}=4 \hat{\mu}_{i}$.

Outliers typically increase MSE. The increase in MSE, compared to the model with no outliers was analyzed with the following ratio: 


$$
M S E_{\text {Ratio }}=\frac{M S E_{A V}}{M S E_{A V}^{\text {out }}}=\frac{\sum_{i=1}^{r} S E_{i}}{\sum_{i=1}^{r} S E_{i}^{\text {out }}} .
$$

In this context, the superscript "out" refers to the model with outliers. The closer $M S E_{\text {Ratio }}$ is to one, the more robust the model is to outliers. $M S E_{\text {Ratio }}$ values close to zero reflect poor performance in the presence of outliers. 


\section{CHAPTER 3}

\section{THE MONTE CARLO SIMULATION}

\subsection{Simulation Technique}

The main focus of this thesis is to compare the performance of different estimators in the presence of moderately to highly correlated independent variables. That means, the independent variables are a result of $\rho$, the coefficient of correlation. The thesis will use $\rho$ values of $0.85,0.90,0.95$, and 0.99 . The independent variables are generated as follows:

$$
x_{i j}=\sqrt{1-\rho^{2}} z_{i j}+\rho z_{i p} ; i=1,2, \ldots, n ; j=1,2, \ldots, p
$$

where $z_{i j}$ are pseudo-random numbers from the standard normal distribution.

The dependent variable (y) is generated in $\mathrm{R}$ using pseudo-random numbers from

the Poisson distribution, with mean $\mu_{i}=e^{\beta_{0}+\beta_{1} x_{i 1}+\ldots+\beta_{p} x_{i p}} ; \mathrm{i}=1,2, \ldots, \mathrm{n}$

The values of $\beta_{j}$ are chosen so that $\sum_{j=1}^{p} \beta_{j}^{2}=1$ and $\beta_{1}=\beta_{2}=\ldots=\beta_{p}$. These are common restrictions in many simulation studies (Månsson and Shukur, 2011).

The degrees of freedom are defined as $d f=n-p-1$. The value of $\beta_{0}$ will vary in this simulation, using the values $\beta_{0}=-1,0,1$. Decreasing the value of $\beta_{0}$ results in a smaller value of $\mu_{i}$, which leads to generating more values equal to zero. If a sample consists of only zeroes, this leads to non-convergence. For each intercept $\left(\beta_{0}\right)$ value, IWLS converges with the following values for $d f$.

$$
\begin{aligned}
& \beta_{0}=1: d f=10,15,20,30,50 \\
& \beta_{0}=0: d f=15,20,30,50,75 \\
& \beta_{0}=-1: d f=30,50,75,100,150
\end{aligned}
$$




\subsection{Simulation Results}

Figure 3.1 shows the MSE of each k estimator at each intercept, for $\rho=.85$. Tables for other correlation values can be found in the Appendix. The tables 3.1 through 3.3 show some of the simulation results. Full simulation results are available in the appendix.

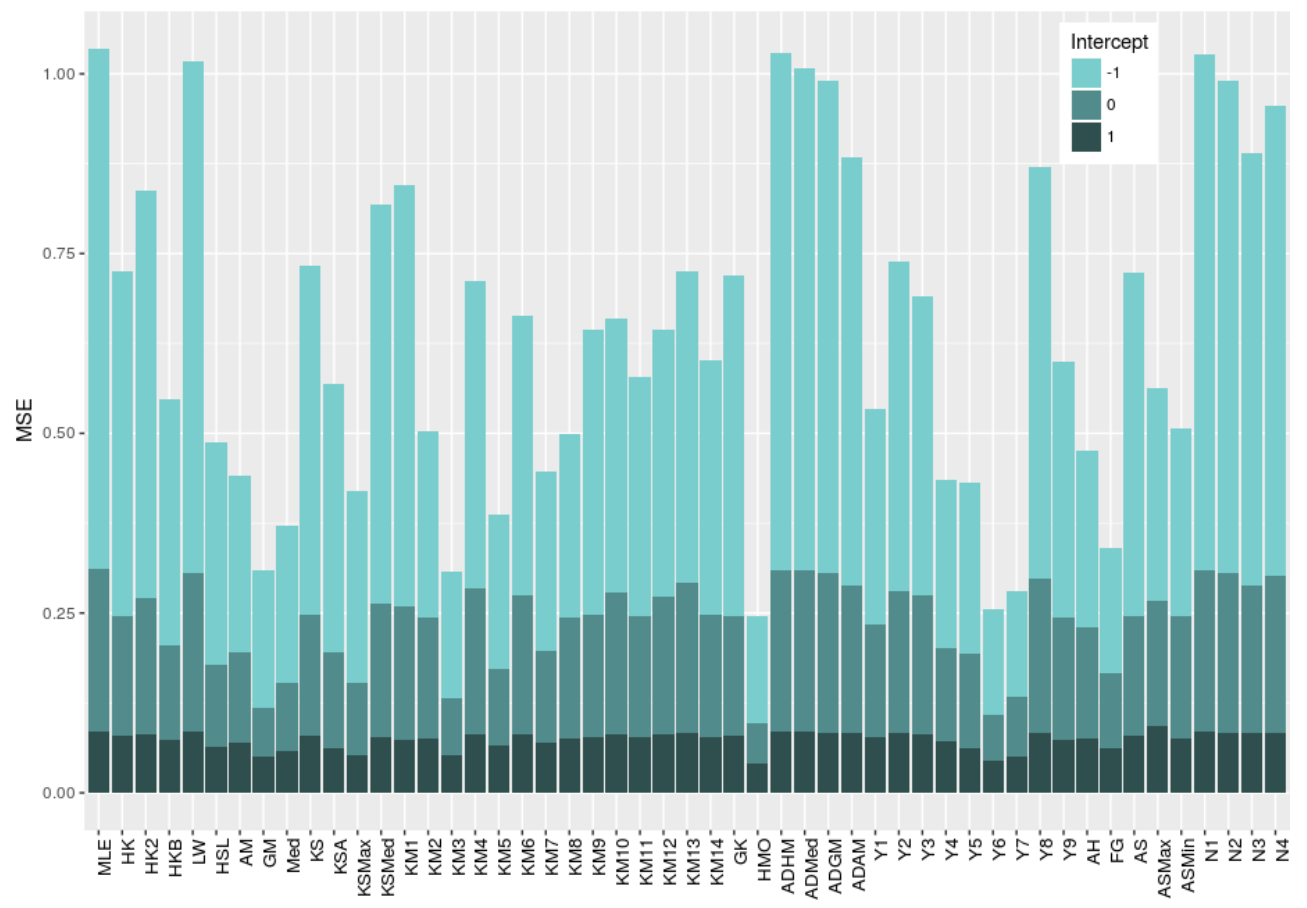

Figure 3.1: MSE for each k estimator, $n=35, \rho=.85$ 
Table 3.1: Poisson Regression Simulation Results, $\mathrm{P}=4, \mathrm{n}=35$, Correlation $(\rho)=.85$

1. Performance $=$ Percentage of times PRR outperforms MLE

\begin{tabular}{|c|c|c|c|c|c|c|c|c|c|}
\hline \multirow[b]{2}{*}{$\mathrm{k}$} & \multicolumn{3}{|c|}{ Intercept $=-1$} & \multicolumn{3}{|c|}{ Intercept $=0$} & \multicolumn{3}{|c|}{ Intercept $=1$} \\
\hline & MSE & Perf. 1 & MAPE & MSE & Perf. & MAPE & MSE & Perf. & MAPE \\
\hline$M L E$ & 0.724 & 0 & 61.922 & 0.226 & 0 & 35.586 & 0.085 & 0 & 21.787 \\
\hline$H K$ & 0.480 & 93 & 51.108 & 0.167 & 92.36 & 30.862 & 0.079 & 80.44 & 21.108 \\
\hline$H K_{2}$ & 0.567 & 93.68 & 55.216 & 0.190 & 92.8 & 32.858 & 0.081 & 80.56 & 21.394 \\
\hline$H K B$ & 0.343 & 92 & 43.856 & 0.132 & 91.64 & 27.628 & 0.073 & 79.96 & 20.406 \\
\hline$L W$ & 0.713 & 94.4 & 61.635 & 0.220 & 93.36 & 35.238 & 0.085 & 81 & 21.785 \\
\hline$H S L$ & 0.309 & 90.96 & 40.885 & 0.115 & 91.12 & 25.740 & 0.063 & 77.48 & 19.189 \\
\hline$A M$ & 0.245 & 73.76 & 41.045 & 0.126 & 73.76 & 27.100 & 0.070 & 63.44 & 19.486 \\
\hline$G M$ & 0.190 & 84.2 & 34.500 & 0.069 & 85.24 & 20.684 & 0.050 & 74.48 & 17.292 \\
\hline Med & 0.220 & 88.76 & 36.036 & 0.093 & 89.84 & 23.406 & 0.059 & 77.16 & 18.633 \\
\hline$K S$ & 0.486 & 93.08 & 51.422 & 0.168 & 92.4 & 30.990 & 0.079 & 80.44 & 21.115 \\
\hline$K S_{A}$ & 0.373 & 92.48 & 45.521 & 0.134 & 91.96 & 27.849 & 0.062 & 75.8 & 18.879 \\
\hline$K S_{\operatorname{Max}}$ & 0.267 & 90.12 & 38.862 & 0.099 & 90.04 & 24.114 & 0.053 & 71.32 & 17.625 \\
\hline$K S_{\text {Med }}$ & 0.554 & 93.72 & 55.388 & 0.187 & 92.96 & 32.781 & 0.077 & 80.2 & 20.833 \\
\hline$K M_{1}$ & 0.586 & 93.76 & 56.312 & 0.185 & 92.88 & 32.433 & 0.074 & 79.72 & 20.467 \\
\hline$K M_{2}$ & 0.259 & 93.36 & 40.208 & 0.167 & 93 & 31.695 & 0.076 & 80.28 & 20.851 \\
\hline$K M_{3}$ & 0.176 & 84.08 & 34.124 & 0.080 & 86.68 & 22.225 & 0.052 & 74.4 & 17.572 \\
\hline$K M_{4}$ & 0.428 & 93.92 & 50.690 & 0.202 & 93.28 & 34.208 & 0.082 & 80.68 & 21.508 \\
\hline$K M_{5}$ & 0.214 & 90.32 & 35.985 & 0.107 & 91.6 & 25.337 & 0.065 & 79.04 & 19.392 \\
\hline$K M_{6}$ & 0.390 & 93.64 & 48.491 & 0.193 & 93.24 & 33.572 & 0.081 & 80.6 & 21.385 \\
\hline$K M_{7}$ & 0.248 & 91.4 & 38.607 & 0.129 & 92.16 & 27.668 & 0.069 & 79.52 & 20.023 \\
\hline$K M_{8}$ & 0.256 & 93.28 & 40.034 & 0.167 & 93 & 31.643 & 0.076 & 80.28 & 20.847 \\
\hline$K M_{9}$ & 0.397 & 93.12 & 47.955 & 0.169 & 92.72 & 31.319 & 0.078 & 80.44 & 21.045 \\
\hline$K M_{10}$ & 0.382 & 93.68 & 48.269 & 0.196 & 93.24 & 33.818 & 0.082 & 80.68 & 21.460 \\
\hline$K M_{11}$ & 0.334 & 93.2 & 44.925 & 0.168 & 92.76 & 31.490 & 0.077 & 80.44 & 20.980 \\
\hline$K M_{12}$ & 0.372 & 93.6 & 47.538 & 0.191 & 93.24 & 33.426 & 0.081 & 80.6 & 21.371 \\
\hline$K M_{13}$ & 0.433 & 94.08 & 51.165 & 0.210 & 93.36 & 34.768 & 0.083 & 80.92 & 21.654 \\
\hline$K M_{14}$ & 0.354 & 93.16 & 45.955 & 0.169 & 92.76 & 31.440 & 0.078 & 80.44 & 21.003 \\
\hline$G K$ & 0.474 & 93 & 50.959 & 0.167 & 92.36 & 30.855 & 0.079 & 80.44 & 21.107 \\
\hline$H M O$ & 0.148 & 81.76 & 31.857 & 0.056 & 80.6 & 19.618 & 0.041 & 59.88 & 15.994 \\
\hline$A D_{H M}$ & 0.718 & 94.4 & 61.721 & 0.225 & 93.36 & 35.561 & 0.085 & 81 & 21.785 \\
\hline$A D_{\text {Med }}$ & 0.699 & 94.28 & 61.030 & 0.224 & 93.36 & 35.483 & 0.085 & 81 & 21.779 \\
\hline$A D_{G M}$ & 0.685 & 94.28 & 60.405 & 0.222 & 93.36 & 35.334 & 0.084 & 81 & 21.771 \\
\hline$A D_{A M}$ & 0.594 & 92.16 & 56.878 & 0.206 & 92.6 & 34.048 & 0.083 & 80.52 & 21.548 \\
\hline$Y_{1}$ & 0.299 & 91.72 & 42.048 & 0.157 & 92.32 & 30.191 & 0.077 & 80.2 & 20.975 \\
\hline$Y_{2}$ & 0.458 & 93.48 & 51.180 & 0.198 & 93.12 & 33.712 & 0.083 & 80.8 & 21.597 \\
\hline$Y_{3}$ & 0.416 & 93.24 & 49.074 & 0.193 & 93 & 33.352 & 0.082 & 80.8 & 21.546 \\
\hline$Y_{4}$ & 0.235 & 89.52 & 38.039 & 0.130 & 91.4 & 27.502 & 0.071 & 79.44 & 20.269 \\
\hline$Y_{5}$ & 0.238 & 92.44 & 38.120 & 0.131 & 92.36 & 28.106 & 0.062 & 77.92 & 18.982 \\
\hline$Y_{6}$ & 0.147 & 83.96 & 31.808 & 0.064 & 87.56 & 19.812 & 0.045 & 70.68 & 16.565 \\
\hline$Y_{7}$ & 0.147 & 88.64 & 30.253 & 0.083 & 90.44 & 22.279 & 0.051 & 75.44 & 17.425 \\
\hline$Y_{8}$ & 0.574 & 94 & 56.748 & 0.213 & 93.32 & 34.760 & 0.084 & 80.92 & 21.680 \\
\hline$Y_{9}$ & 0.356 & 93.28 & 45.921 & 0.171 & 93 & 31.707 & 0.073 & 79.72 & 20.347 \\
\hline$A H$ & 0.245 & 92.72 & 38.736 & 0.154 & 92.76 & 30.378 & 0.076 & 80.4 & 20.785 \\
\hline$F G$ & 0.174 & 90.72 & 33.024 & 0.105 & 91.68 & 25.449 & 0.062 & 78.56 & 19.074 \\
\hline$A S$ & 0.477 & 93 & 51.031 & 0.167 & 92.36 & 30.859 & 0.079 & 80.44 & 21.108 \\
\hline$A S_{\operatorname{Max}}$ & 0.295 & 67.32 & 46.471 & 0.174 & 65 & 32.004 & 0.093 & 56.24 & 21.985 \\
\hline$A S_{M i n}$ & 0.262 & 93.44 & 40.257 & 0.170 & 93.12 & 31.983 & 0.075 & 80.2 & 20.756 \\
\hline$N_{1}$ & 0.717 & 94.36 & 61.698 & 0.225 & 93.36 & 35.558 & 0.085 & 81 & 21.785 \\
\hline $\mathrm{N}_{2}$ & 0.685 & 94.24 & 60.485 & 0.221 & 93.36 & 35.254 & 0.084 & 80.96 & 21.758 \\
\hline$N_{3}$ & 0.602 & 93.92 & 56.995 & 0.205 & 93.2 & 34.033 & 0.083 & 80.84 & 21.615 \\
\hline$N_{4}$ & 0.654 & 94.08 & 59.318 & 0.217 & 93.32 & 34.968 & 0.084 & 80.96 & 21.732 \\
\hline
\end{tabular}


Table 3.2: Poisson Regression Simulation Results, $\mathrm{P}=4, \mathrm{n}=600, \rho=.85$

\begin{tabular}{|c|c|c|c|c|c|c|c|c|c|}
\hline \multirow[b]{2}{*}{$\mathrm{k}$} & \multicolumn{3}{|c|}{ Intercept $=-1$} & \multicolumn{3}{|c|}{ Intercept $=0$} & \multicolumn{3}{|c|}{ Intercept $=1$} \\
\hline & MSE & Perf. & MAPE & MSE & Perf. & MAPE & MSE & Perf. & MAPE \\
\hline$M L E$ & 0.01150986 & 0 & 8.512063 & 0.004234872 & 0 & 5.121368 & 0.001543436 & 0 & 3.098929 \\
\hline$H K$ & 0.01138037 & 63.24 & 8.46579 & 0.004207128 & 54.72 & 5.105159 & 0.001541863 & 53.36 & 3.097465 \\
\hline$H K_{2}$ & 0.01144787 & 63.68 & 8.490171 & 0.004220655 & 55.08 & 5.113087 & 0.001542557 & 53.44 & 3.098101 \\
\hline$H K B$ & 0.01127771 & 62.44 & 8.430031 & 0.004181141 & 54.4 & 5.089842 & 0.001540023 & 53.24 & 3.095712 \\
\hline$L W$ & 0.01150984 & 64.08 & 8.512056 & 0.004234812 & 55.16 & 5.121334 & 0.001543436 & 53.48 & 3.098929 \\
\hline$H S L$ & 0.01123679 & 61.68 & 8.416628 & 0.004169296 & 53.92 & 5.081385 & 0.001534335 & 52.48 & 3.090826 \\
\hline$A M$ & 0.03475409 & 39.56 & 12.721099 & 0.01681423 & 40.2 & 7.742635 & 0.008378082 & 40.44 & 4.529795 \\
\hline$G M$ & 0.01142872 & 55.2 & 8.48663 & 0.004286356 & 49.72 & 5.153869 & 0.001570687 & 50 & 3.108378 \\
\hline Med & 0.01115915 & 60.48 & 8.392031 & 0.004134738 & 52.92 & 5.064132 & 0.001531667 & 52.32 & 3.08872 \\
\hline$K S$ & 0.01138331 & 63.24 & 8.466812 & 0.004207558 & 54.72 & 5.105391 & 0.001541867 & 53.36 & 3.097469 \\
\hline$K S_{A}$ & 0.01133133 & 62.68 & 8.451013 & 0.004198166 & 54.04 & 5.102479 & 0.001542575 & 51.28 & 3.097779 \\
\hline$K S_{\text {Max }}$ & 0.01132355 & 60.52 & 8.456149 & 0.00423504 & 52.6 & 5.133492 & 0.001709692 & 46.76 & 3.218501 \\
\hline$K S_{\text {Med }}$ & 0.0114438 & 63.68 & 8.488287 & 0.00421745 & 54.88 & 5.111084 & 0.001539763 & 53.16 & 3.095327 \\
\hline$K M_{1}$ & 0.01144208 & 63.56 & 8.487929 & 0.004212198 & 54.72 & 5.107901 & 0.001537675 & 53 & 3.093564 \\
\hline$K M_{2}$ & 0.01137574 & 63.2 & 8.464738 & 0.004223419 & 55.08 & 5.114695 & 0.001541466 & 53.28 & 3.097074 \\
\hline$K M_{3}$ & 0.01294655 & 53.92 & 8.877511 & 0.004527218 & 50.72 & 5.275558 & 0.001812893 & 49.48 & 3.197153 \\
\hline $\mathrm{KM}_{4}$ & 0.0114616 & 63.84 & 8.495074 & 0.004231371 & 55.16 & 5.119295 & 0.001542867 & 53.44 & 3.098404 \\
\hline$K M_{5}$ & 0.01114499 & 61.56 & 8.382635 & 0.00416452 & 54.28 & 5.080265 & 0.00153611 & 52.92 & 3.09183 \\
\hline$K M_{6}$ & 0.01143557 & 63.6 & 8.48553 & 0.004229437 & 55.16 & 5.118169 & 0.001542666 & 53.44 & 3.098202 \\
\hline$K M_{7}$ & 0.01126475 & 62.4 & 8.427818 & 0.004188605 & 54.56 & 5.094419 & 0.001538607 & 53.04 & 3.094519 \\
\hline$K M_{8}$ & 0.01137431 & 63.2 & 8.464252 & 0.004223336 & 55.08 & 5.114648 & 0.001541463 & 53.28 & 3.097072 \\
\hline$K M_{9}$ & 0.01138219 & 63.24 & 8.466605 & 0.004214608 & 54.8 & 5.109547 & 0.001541768 & 53.32 & 3.097373 \\
\hline$K M_{10}$ & 0.01145188 & 63.72 & 8.491651 & 0.004230782 & 55.16 & 5.118969 & 0.001542822 & 53.44 & 3.09836 \\
\hline$K M_{11}$ & 0.01138017 & 63.2 & 8.466048 & 0.004218564 & 55.04 & 5.111862 & 0.001541672 & 53.32 & 3.097279 \\
\hline$K M_{12}$ & 0.01143267 & 63.6 & 8.484558 & 0.004229247 & 55.16 & 5.118063 & 0.001542658 & 53.44 & 3.098195 \\
\hline$K M_{13}$ & 0.01148367 & 63.92 & 8.5029 & 0.004233733 & 55.16 & 5.120697 & 0.001543209 & 53.48 & 3.098721 \\
\hline$K M_{14}$ & 0.01138096 & 63.24 & 8.466272 & 0.004217352 & 54.88 & 5.111153 & 0.001541706 & 53.32 & 3.097313 \\
\hline$G K$ & 0.01138036 & 63.24 & 8.465787 & 0.004207127 & 54.72 & 5.105159 & 0.001541863 & 53.36 & 3.097465 \\
\hline$H M O$ & 0.06170781 & 1.44 & 21.577229 & 0.029389854 & 0.12 & 16.474444 & 0.025302398 & 0 & 10.214126 \\
\hline$A D_{H M}$ & 0.01150984 & 64.08 & 8.512056 & 0.004234871 & 55.16 & 5.121367 & 0.001543436 & 53.48 & 3.098929 \\
\hline$A D_{M e d}$ & 0.01150981 & 64.08 & 8.512045 & 0.004234867 & 55.16 & 5.121365 & 0.001543436 & 53.48 & 3.098929 \\
\hline$A D_{G M}$ & 0.0115097 & 64.08 & 8.512004 & 0.004234855 & 55.16 & 5.121358 & 0.001543435 & 53.48 & 3.098928 \\
\hline$A D_{A M}$ & 0.01160264 & 63.84 & 8.533477 & 0.004246769 & 55.16 & 5.125136 & 0.001580016 & 53.4 & 3.110466 \\
\hline$Y_{1}$ & 0.01147738 & 63.48 & 8.498829 & 0.004229116 & 55.08 & 5.117988 & 0.001545904 & 53.4 & 3.099343 \\
\hline$Y_{2}$ & 0.0115023 & 64.04 & 8.509383 & 0.004234152 & 55.16 & 5.12096 & 0.001543396 & 53.48 & 3.098891 \\
\hline$Y_{3}$ & 0.01149518 & 64 & 8.506978 & 0.004233192 & 55.16 & 5.120417 & 0.001543364 & 53.48 & 3.098861 \\
\hline$Y_{4}$ & 0.01150995 & 62.88 & 8.512104 & 0.004224116 & 54.96 & 5.114994 & 0.001558972 & 53.36 & 3.101794 \\
\hline$Y_{5}$ & 0.01068924 & 56.8 & 8.222426 & 0.004097358 & 53.56 & 5.037657 & 0.001522528 & 48.92 & 3.077115 \\
\hline$Y_{6}$ & 0.02257348 & 18.8 & 12.373545 & 0.005245744 & 33.88 & 5.844202 & 0.002009344 & 34.04 & 3.486024 \\
\hline$Y_{7}$ & 0.01181426 & 42.04 & 8.721614 & 0.003995929 & 47.48 & 4.9773 & 0.001565748 & 44.16 & 3.121525 \\
\hline$Y_{8}$ & 0.01150566 & 64.04 & 8.510631 & 0.004234499 & 55.16 & 5.121156 & 0.001543401 & 53.48 & 3.098895 \\
\hline$Y_{9}$ & 0.01096208 & 60.56 & 8.319336 & 0.004159612 & 54.04 & 5.075922 & 0.001524806 & 51.64 & 3.082006 \\
\hline$A H$ & 0.0113114 & 62.8 & 8.441103 & 0.004214862 & 54.92 & 5.109691 & 0.001541351 & 53.28 & 3.096957 \\
\hline$F G$ & 0.01098807 & 60.88 & 8.326171 & 0.004167353 & 54.32 & 5.081613 & 0.001535437 & 52.96 & 3.091333 \\
\hline$A S$ & 0.01138036 & 63.24 & 8.465789 & 0.004207128 & 54.72 & 5.105159 & 0.001541863 & 53.36 & 3.097465 \\
\hline$A S_{\operatorname{Max}}$ & 0.05602494 & 29.88 & 16.310353 & 0.029175583 & 32.08 & 10.057508 & 0.014185851 & 33.12 & 5.722722 \\
\hline$A S_{\text {Min }}$ & 0.01137061 & 63.32 & 8.463101 & 0.00422612 & 55.16 & 5.116259 & 0.001541251 & 53.28 & 3.096858 \\
\hline$N_{1}$ & 0.01150984 & 64.08 & 8.512055 & 0.00423487 & 55.16 & 5.121367 & 0.001543436 & 53.48 & 3.098929 \\
\hline$N_{2}$ & 0.01150832 & 64.08 & 8.511526 & 0.004234663 & 55.16 & 5.121249 & 0.001543428 & 53.48 & 3.098921 \\
\hline$N_{3}$ & 0.01149267 & 63.96 & 8.506032 & 0.004231828 & 55.16 & 5.119603 & 0.001543289 & 53.48 & 3.09879 \\
\hline$N_{4}$ & 0.01150685 & 64.08 & 8.511014 & 0.004234464 & 55.16 & 5.121135 & 0.001543421 & 53.48 & 3.098914 \\
\hline
\end{tabular}


Table 3.3: MSE Ratios (10\% Outliers), $\mathrm{P}=4, \mathrm{n}=35$

\begin{tabular}{|c|c|c|c|c|c|c|c|c|c|c|c|c|}
\hline \multirow[b]{2}{*}{$\mathrm{k}$} & \multicolumn{3}{|c|}{$\rho=.85$} & \multicolumn{3}{|c|}{$\rho=.90$} & \multicolumn{3}{|c|}{$\rho=.95$} & \multicolumn{3}{|c|}{$\rho=.99$} \\
\hline & -1 & 0 & 1 & -1 & 0 & 1 & -1 & 0 & 1 & -1 & 0 & 1 \\
\hline$M L E$ & 0.548 & 0.236 & 0.094 & 0.524 & 0.228 & 0.091 & 0.505 & 0.223 & 0.086 & 0.518 & 0.218 & 0.086 \\
\hline$H K$ & .469 & 0.192 & 0.089 & 0.427 & 0.176 & 0.084 & 0.384 & 0.155 & 0.074 & 0.338 & 0.122 & 0.053 \\
\hline$H K_{2}$ & 0.497 & 0.21 & 0.091 & 0.46 & 0.196 & 0.087 & 0.421 & 0.179 & 0.079 & 0.392 & 0.151 & 0.064 \\
\hline$H K B$ & 0.407 & 0.166 & 0.085 & 0.358 & 0.146 & 0.078 & 0.306 & 0.12 & 0.064 & 0.258 & 0.086 & 0.038 \\
\hline$L W$ & 0.547 & 0.232 & 0.094 & 0.521 & 0.221 & 0.091 & 0.5 & 0.209 & 0.086 & 0.49 & 0.179 & 0.086 \\
\hline$H S L$ & 0.424 & 0.17 & 0.079 & 0.359 & 0.142 & 0.069 & 0.316 & 0.113 & 0.047 & 0.258 & 0.075 & 0.022 \\
\hline$A M$ & 0.628 & 0.296 & 0.138 & 0.52 & 0.207 & 0.097 & 0.353 & 0.121 & 0.053 & 0.144 & 0.039 & 0.016 \\
\hline$G M$ & 0.38 & 0.13 & 0.071 & 0.303 & 0.098 & 0.056 & 0.219 & 0.061 & 0.037 & 0.194 & 0.044 & 0.017 \\
\hline Med & 0.366 & 0.142 & 0.077 & 0.304 & 0.12 & 0.065 & 0.24 & 0.088 & 0.048 & 0.2 & 0.057 & 0.025 \\
\hline$K S$ & 0.471 & 0.193 & 0.089 & 0.43 & 0.177 & 0.084 & 0.386 & 0.156 & 0.074 & 0.339 & 0.122 & 0.053 \\
\hline$K S_{A}$ & 0.465 & 0.193 & 0.082 & 0.418 & 0.176 & 0.072 & 0.383 & 0.148 & 0.054 & 0.307 & 0.104 & 0.035 \\
\hline$K S_{\text {Max }}$ & 0.441 & 0.182 & 0.082 & 0.385 & 0.154 & 0.066 & 0.336 & 0.121 & 0.044 & 0.234 & 0.074 & 0.022 \\
\hline$K S_{\text {Med }}$ & 0.509 & 0.216 & 0.09 & 0.481 & 0.208 & 0.085 & 0.462 & 0.197 & 0.078 & 0.461 & 0.182 & 0.069 \\
\hline$K M_{1}$ & 0.522 & 0.216 & 0.087 & 0.495 & 0.207 & 0.083 & 0.476 & 0.198 & 0.074 & 0.479 & 0.184 & 0.066 \\
\hline$K M_{2}$ & 0.398 & 0.217 & 0.093 & 0.365 & 0.215 & 0.088 & 0.335 & 0.21 & 0.085 & 0.294 & 0.201 & 0.098 \\
\hline$K M_{3}$ & 449 & 0.167 & 0.082 & 0.375 & 0.139 & 0.068 & 0.277 & 0.097 & 0.048 & .136 & 0.044 & 0.022 \\
\hline$K M_{4}$ & 0.465 & 0.229 & 0.094 & 0.434 & 0.229 & 0.091 & 0.413 & 0.226 & 0.089 & 0.381 & 0.242 & 0.111 \\
\hline$K M_{5}$ & 356 & 0.157 & 0.081 & 0.298 & 0.139 & 0.072 & 0.238 & 0.106 & & .152 & 0.057 & 0.028 \\
\hline$K M_{6}$ & 0.447 & 0.225 & 0.094 & 0.418 & 0.223 & 0.09 & 0.395 & 0.218 & 0.087 & 0.346 & 0.218 & 0.101 \\
\hline$K M_{7}$ & .37 & 0.173 & 0.085 & 0.316 & 0.157 & 0.076 & 0.258 & 0.126 & & 0.169 & 0.069 & 0.035 \\
\hline$K M_{8}$ & 0.397 & 0.216 & 0.093 & 0.363 & 0.215 & 0.088 & 0.334 & 0.209 & 0.085 & 0.293 & 0.2 & 0.097 \\
\hline$K M_{9}$ & 436 & 0.198 & 0.09 & 0.389 & 0.184 & 0.085 & 0.337 & 0.159 & & 0.236 & 0.101 & 0.049 \\
\hline$K M_{10}$ & 0.438 & 0.225 & 0.094 & 0.406 & 0.224 & 0.09 & 0.383 & 0.219 & 0.088 & 0.341 & 0.228 & 0.108 \\
\hline$K M_{11}$ & 0.412 & 0.203 & 0.09 & 0.367 & 0.193 & 0.085 & 0.317 & 0.169 & 0.076 & 0.212 & 0.109 & 0.054 \\
\hline$K M_{12}$ & 0.438 & 0.224 & 0.094 & 0.406 & 0.221 & 0.09 & 0.383 & 0.215 & 0.087 & 0.333 & 0.214 & 0.1 \\
\hline$K M_{13}$ & 0.462 & 0.234 & 0.095 & 0.44 & 0.237 & 0.092 & 0.441 & 0.246 & 0.094 & 0.494 & 0.356 & 0.164 \\
\hline$K M_{14}$ & 0.419 & 0.201 & 0.09 & 0.373 & 0.189 & 0.085 & 0.321 & 0.165 & 0.075 & 0.214 & 0.103 & 0.051 \\
\hline$G K$ & 0.467 & 0.192 & 0.089 & 0.424 & 0.176 & 0.084 & 0.381 & 0.155 & 0.074 & 0.322 & 0.121 & 0.053 \\
\hline$H M O$ & 0.583 & 0.293 & 0.158 & 0.475 & 0.191 & 0.105 & 0.295 & 0.091 & 0.05 & 0.17 & 0.042 & 0.016 \\
\hline$A D_{H M}$ & 0.546 & 0.235 & 0.094 & 0.522 & 0.227 & 0.091 & 0.501 & 0.223 & 0.086 & 0.512 & 0.217 & 0.086 \\
\hline$A D_{M e d}$ & 0.541 & 0.235 & 0.094 & & 0.226 & 0.091 & 0.493 & 0.221 & & .501 & 0.215 & 0.085 \\
\hline$A D_{G M}$ & 0.534 & 0.233 & 0.094 & 0.505 & 0.225 & 0.091 & 0.488 & 0.219 & 86 & 0.497 & 0.213 & 0.085 \\
\hline$A D_{A M}$ & 0.495 & & & & & & 446 & & & & & 0.081 \\
\hline$Y_{1}$ & 0.374 & 0.189 & 0.09 & 0.315 & 0.173 & 0.0 & 0.245 & 38 & 72 & 0.117 & 0.063 & 0.041 \\
\hline$Y_{2}$ & & & & & & & & & & & & 0.06 \\
\hline$Y_{3}$ & .425 & 0.212 & 0.092 & 0.371 & 0.198 & 0.088 & 0.296 & 0.172 & & 0.153 & 0.087 & 0.055 \\
\hline$Y_{4}$ & 0.365 & & & 0.308 & & 0.078 & 0.229 & & & 06 & 48 & 0.031 \\
\hline$Y_{5}$ & 0.441 & 0.225 & 0.096 & 0.412 & 0.223 & 0.091 & 0.421 & 0.227 & 89 & 0.427 & 0.259 & 0.114 \\
\hline$Y_{6}$ & 0.722 & 0.283 & 0.133 & 0.718 & 0.279 & 0.135 & 0.748 & 0.276 & 0.143 & 0.993 & 0.365 & 0.218 \\
\hline$Y_{7}$ & 0.513 & 0.232 & 0.109 & 0.495 & 0.234 & 0.108 & 0.494 & 0.241 & 0.115 & 0.629 & 0.299 & 0.181 \\
\hline$Y_{8}$ & 0.495 & 0.226 & 0.093 & 0.453 & 0.216 & 0.09 & 0.411 & 0.201 & 0.083 & 0.328 & 0.163 & 0.073 \\
\hline$Y_{9}$ & 0.477 & 0.232 & 0.094 & 0.465 & 0.233 & 0.092 & 0.459 & 0.237 & 0.092 & 0.439 & 0.242 & 0.111 \\
\hline$A H$ & 0.399 & 0.206 & 0.093 & 0.365 & 0.204 & 0.088 & 0.331 & 0.194 & 0.085 & 0.292 & 0.182 & 0.1 \\
\hline$F G$ & 0.38 & 0.179 & 0.085 & 0.337 & 0.169 & 0.078 & 0.299 & 0.148 & 0.066 & 0.23 & 0.109 & 0.051 \\
\hline$A S$ & 0.468 & 0.192 & 0.089 & 0.426 & 0.176 & 0.084 & 0.382 & 0.155 & 0.074 & 0.329 & 0.122 & 0.053 \\
\hline$A S_{\operatorname{Max}}$ & 0.82 & 0.495 & 0.224 & 0.757 & 0.356 & 0.155 & 0.562 & 0.226 & 0.08 & 0.414 & 0.07 & 0.022 \\
\hline$A S_{\text {Min }}$ & 0.429 & 0.233 & 0.097 & 0.414 & 0.238 & 0.093 & 0.415 & 0.255 & 0.097 & 0.471 & 0.335 & 0.162 \\
\hline$N_{1}$ & 0.546 & 0.235 & 0.094 & 0.521 & 0.227 & 0.091 & 0.501 & 0.223 & 0.086 & 0.512 & 0.217 & 0.086 \\
\hline$N_{2}$ & 0.535 & 0.232 & 0.094 & 0.508 & 0.223 & 0.091 & 0.483 & 0.216 & 0.086 & 0.482 & 0.206 & 0.084 \\
\hline$N_{3}$ & 0.507 & 0.22 & 0.093 & 0.47 & 0.208 & 0.089 & 0.431 & 0.193 & 0.082 & 0.397 & 0.166 & 0.072 \\
\hline$N_{4}$ & 0.525 & 0.229 & 0.094 & 0.495 & 0.219 & 0.09 & 0.467 & 0.211 & 0.085 & 0.456 & 0.198 & 0.082 \\
\hline
\end{tabular}




\subsection{Performance as a Function of $\beta_{0}$}

Simulations were varied by intercept value. The intercept values included were $\beta_{0}=$ $-1,0,1$. Performance, defined as the percentage of times that the estimator had a smaller MSE than the maximum likelihood model, decreased with an increase in intercept value. Both MAPE and MSE decreased with an increase in intercept value, which implies higher accuracy. The intercept value of $\beta_{0}=1$ produced the lowest MSE values.

\subsection{Performance as a Function of $\rho$}

Simulations were varied by correlation. The coefficient of correlation values used were $\rho=.85, .90, .95, .99$. Larger correlation values increased performance percentage, but also increased MSE and MAPE values, with the largest values at $\rho=.99$.

\subsection{Performance as a Function of $n$}

Simulations were varied by sample size $(n)$. Simulations were run using the sample size $n=35$ and $n=600$. Generally, the larger sample size produced much smaller MSE values. It is also notable that the larger sample size affected the performance of individual k estimators. With $n=35$, the k estimators with the lowest MSE values were $Y_{6}, Y_{7}$, $H M O, F G, K M_{2}, K M_{3}, A M, A H, K M_{2}, K M_{8}, A S_{\text {Min }}$, and $A S_{\text {Max }}$. With $n=600$, the $\mathrm{k}$ estimators with the lowest MSE values were $F G, Y_{9}, G M, H S L, M e d$, and $Y_{5}$.

\subsection{Best Performing Estimators}

Estimators were judged by their MSE value at both sample sizes, and performance with outliers. The k estimators that produced the lowest MSE values were selected. Performance with outliers was considered secondarily. The k estimators which produced the smallest MSE values are as follows: $F G, Y_{5}, Y_{9}, A H, K M_{2}$, and $K M_{8}$. 


\subsection{Proposed New Estimators}

The following new estimators are proposed based on the seven best performing $\mathrm{k}$ estimators:

1. $C Z_{1}=$ HarmonicMean $\left\{F G, Y_{5}, Y_{9}, A H, K M_{2}, K M_{8}\right\}$

2. $C Z_{2}=$ GeometricMean $\left\{F G, Y_{5}, Y_{9}, A H, K M_{2}, K M_{8}\right\}$

3. $C Z_{3}=$ ArithmeticMean $\left\{F G, Y_{5}, Y_{9}, A H, K M_{2}, K M_{8}\right\}$

4. $C Z_{4}=$ Median $\left\{F G, Y_{5}, Y_{9}, A H, K M_{2}, K M_{8}\right\}$

5. $C Z_{5}=\operatorname{Maximum}\left\{F G, Y_{5}, Y_{9}, A H, K M_{2}, K M_{8}\right\}$

\subsection{Performance of New Estimators}

Simulations were then run again using these five new estimators. All five estimators performed fairly well. The best performing estimators were $C Z_{1}, C Z_{5}$, and $C Z_{3}$. $C Z_{1}$ frequently had a much smaller MSE than all other estimators. Figure 3.2 shows the MSE for the 15 best performing estimators $\left(\mathrm{n}=35, \rho=.85, \beta_{0}=-1\right)$. See Tables 3.4 and 3.5 and tables A7 through A12 for simulation results with the new estimators. 
Table 3.4: Poisson Regression Simulation Results (New Estimators), $\mathrm{P}=4, \mathrm{n}=35, \rho=.85$

\begin{tabular}{|c|c|c|c|c|c|c|c|c|c|}
\hline \multirow[b]{2}{*}{$\mathrm{k}$} & \multicolumn{3}{|c|}{ Intercept $=-1$} & \multicolumn{3}{|c|}{ Intercept $=0$} & \multicolumn{3}{|c|}{ Intercept $=1$} \\
\hline & MSE & Perf. & MAPE & MSE & Perf. & MAPE & MSE & Perf. & MAPE \\
\hline$M L E$ & 0.7088039 & 0 & 62.29505 & 0.23496786 & 0 & 36.45803 & 0.08247555 & 0 & 21.63941 \\
\hline$H K$ & 0.480297 & 93.88 & 51.40929 & 0.17094316 & 93.28 & 31.42677 & 0.07678822 & 80.36 & 20.98787 \\
\hline$H K_{2}$ & 0.5602051 & 94.32 & 55.57943 & 0.19655611 & 93.8 & 33.56656 & 0.07922176 & 80.6 & 21.26998 \\
\hline$H K B$ & 0.3428405 & 92.6 & 43.95846 & 0.13468219 & 92.36 & 28.14434 & 0.0713326 & 79.56 & 20.32497 \\
\hline$L W$ & 0.7010262 & 95.28 & 62.03473 & 0.22952308 & 94 & 36.10883 & 0.08246094 & 80.84 & 21.63805 \\
\hline$H S L$ & 0.3031443 & 91.64 & 40.74378 & 0.12122675 & 91.32 & 26.40869 & 0.06166002 & 76.96 & 18.99735 \\
\hline$A M$ & 0.2329765 & 76.44 & 39.89231 & 0.11587944 & 75.36 & 26.28239 & 0.06998363 & 64.72 & 19.41486 \\
\hline$G M$ & 0.181186 & 85.52 & 33.60488 & 0.0704999 & 86.44 & 21.06886 & 0.04945014 & 73.96 & 17.26303 \\
\hline Med & 0.2167196 & 89.24 & 35.78722 & 0.09771431 & 90.04 & 24.12621 & 0.0589686 & 77.36 & 18.64888 \\
\hline$K S$ & 0.4859627 & 93.96 & 51.73366 & 0.17250713 & 93.44 & 31.55979 & 0.076843 & 80.36 & 20.99371 \\
\hline$K S_{A}$ & 0.3694238 & 93.12 & 45.71068 & 0.13954408 & 92.4 & 28.43206 & 0.05979188 & 76.84 & 18.6792 \\
\hline$K S_{\operatorname{Max}}$ & 0.2609286 & 91.16 & 38.70245 & 0.10474635 & 90.04 & 24.79633 & 0.05140393 & 71.96 & 17.39264 \\
\hline$K S_{M e d}$ & 0.552789 & 94.44 & 55.83772 & 0.19488102 & 93.8 & 33.52551 & 0.07478304 & 80 & 20.72871 \\
\hline$K M_{1}$ & 0.5799505 & 94.56 & 56.6908 & 0.19231507 & 93.72 & 33.1996 & 0.07199818 & 79.36 & 20.35179 \\
\hline$K M_{2}$ & 0.2580438 & 93.64 & 40.19466 & 0.17640125 & 93.8 & 32.43258 & 0.07475317 & 80.12 & 20.77511 \\
\hline$K M_{3}$ & 0.1705738 & 85.52 & 33.35542 & 0.08165053 & 88.08 & 22.45 & 0.05130315 & 74.12 & 17.52435 \\
\hline$K M_{4}$ & 0.433351 & 94.8 & 51.20969 & 0.21112566 & 93.96 & 34.99528 & 0.08006497 & 80.68 & 21.3766 \\
\hline$K M_{5}$ & 0.2110569 & 91.08 & 35.63777 & 0.11213641 & 91.88 & 25.9181 & 0.06375041 & 78.96 & 19.34943 \\
\hline$K M_{6}$ & 3910307 & 94.32 & 48.77392 & 0.20137321 & 93.92 & 34.31872 & 0.07893522 & 80.6 & 21.25507 \\
\hline$K M_{7}$ & 0.2481702 & 92.36 & 38.53884 & 0.13514549 & 92.68 & 28.3034 & 0.06853487 & 79.44 & 19.98591 \\
\hline$K M_{8}$ & 0.2557311 & 93.6 & 40.01769 & 0.17572959 & 93.8 & 32.3795 & 0.07472062 & 80.12 & 20.77165 \\
\hline$K M_{9}$ & 4000639 & 94.08 & 48.30235 & 0.17439656 & 93.64 & 31.941 & 0.07629392 & 80.32 & 20.93768 \\
\hline$K M_{10}$ & 3857255 & 94.48 & 48.67987 & 0.20540942 & 93.96 & 34.61514 & 0.07969724 & 80.68 & 21.33783 \\
\hline$K M_{11}$ & 3363765 & 93.92 & 45.1 & 0.17522211 & 93.8 & 498 & 0.07578451 & 80.24 & 20.8 \\
\hline$K M_{12}$ & 0.374274 & 94.32 & 47.8 & 39294 & 93.92 & 779 & 0.07883548 & 80.6 & 21.24 \\
\hline$K M_{13}$ & 0.4374917 & 94.92 & 51.68184 & 0.22003256 & 94 & 622 & 0.08131545 & 80.84 & 21.51728 \\
\hline$K M_{14}$ & 3566891 & 93.96 & 46.2593 & 0.17499776 & 93.72 & 32.09033 & 0.07596436 & 80.24 & 20.90345 \\
\hline$G K$ & 4766073 & 93.84 & 51.28546 & 0.17084909 & 93.28 & 2034 & 0.0767832 & 80.36 & 20.98736 \\
\hline HМO & 0.1471305 & 82.8 & 31.61548 & 0.05709397 & 80.92 & 2464 & 0.04045745 & 60.76 & 15.80 \\
\hline$A D_{H M}$ & 0.70388 & 95.28 & 62.1 & 5228 & 94 & 172 & 0.08246418 & 80.84 & 3829 \\
\hline$A D_{\text {Med }}$ & 0.6878477 & 95.24 & 61.4 & 7039 & 94 & 625 & 242619 & 80.84 & 3442 \\
\hline$A D_{G M}$ & 6741254 & 95.12 & 60.88254 & 3067 & 94 & 32 & 235703 & 80.84 & 21.6 \\
\hline$A D_{A M}$ & 5834015 & 93.24 & 56.93001 & 0.21807692 & 93.36 & 2643 & 08089365 & 80.52 & 21.44064 \\
\hline$Y_{1}$ & 0.2935828 & 92.48 & 41.82392 & 0.16556772 & 93.12 & 31.04446 & 0.07627836 & 80.48 & 20.93689 \\
\hline$Y_{2}$ & 4554355 & 94.32 & 51.55713 & 0.20672677 & 93.84 & 34.52761 & 0.08089034 & 80.84 & 21.47094 \\
\hline$Y_{3}$ & 4127159 & 94.2 & 49.18808 & 0.20015709 & 93.76 & 6478 & 0.08048913 & 80.8 & 21.42603 \\
\hline$Y_{4}$ & 2280989 & 90.24 & 37.3 & 0623 & 91.92 & 225 & 657 & 79.72 & 20.26871 \\
\hline$Y_{5}$ & 2386109 & 92.92 & 38.27941 & 0.13788402 & 93.2 & 409 & 0.06085483 & 77.84 & 18.91436 \\
\hline$Y_{6}$ & 1493494 & 83.52 & 32.00474 & 0.06747108 & 88.36 & 20.31241 & 0.0444064 & 70.2 & 16.50523 \\
\hline$Y_{7}$ & 1494385 & 88.84 & 30.3728 & 0.08726362 & 91.2 & 22.76762 & 0.05023304 & 74.92 & 17.36867 \\
\hline$Y_{8}$ & 5746807 & 94.92 & 57.15903 & 0.22168357 & 93.96 & 35.59712 & 0.08158783 & 80.84 & 21.5436 \\
\hline$Y_{9}$ & 3643156 & 93.92 & 46.33327 & 0.17723258 & 93.72 & 32.34682 & 0.07107182 & 79.68 & 20.22165 \\
\hline$A H$ & 2459471 & 93.32 & 38.73002 & 0.16063297 & 93.68 & 30.97715 & 0.0741905 & 80.16 & 20.71211 \\
\hline$F G$ & 1730899 & 90.92 & 32.80834 & 0.11048376 & 92.32 & 25.99092 & 0.06127913 & 78.64 & 19.03669 \\
\hline$A S$ & 4784081 & 93.84 & 51.34638 & 0.17089566 & 93.28 & 31.42352 & 0.07678569 & 80.36 & 20.98761 \\
\hline & 2856356 & 69.16 & 45.54903 & 16075411 & 65.92 & 7024 & 0.09155866 & 58.04 & 21.6205 \\
\hline$A S_{\text {Min }}$ & 0.2620687 & 93.88 & & 0.17957039 & 93.8 & & 0.0740133 & 80.08 & 20.69343 \\
\hline$N_{1}$ & 0.7033129 & 95.28 & 62.09097 & 0.23450362 & 94 & 36.42864 & 0.08246284 & 80.84 & 21.63816 \\
\hline$N_{2}$ & 0.6741733 & 95.16 & 60.89254 & 0.22975064 & 94 & 36.10502 & 0.08223508 & 80.88 & 21.61395 \\
\hline$N_{3}$ & 5955888 & 94.64 & 57.39412 & 0.21194221 & 93.88 & 34.80084 & 0.08102742 & 80.84 & 21.47984 \\
\hline$N_{4}$ & 6466877 & 94.96 & 59.73238 & 0.22533975 & 94 & 35.79882 & 0.08202104 & 80.84 & 21.59104 \\
\hline$C Z_{1}$ & 0.1407474 & 87.68 & 30.11826 & 0.07844236 & 91.32 & 22.04534 & 0.04887127 & 75.4 & 17.19815 \\
\hline$C Z_{2}$ & 0.2586082 & 93.32 & 40.06886 & 0.16161437 & 93.64 & 31.11928 & 0.07158889 & 79.88 & 20.37312 \\
\hline$C Z_{3}$ & 0.2306893 & 93.04 & 37.87982 & 0.14956652 & 93.48 & 30.00945 & 0.06836374 & 79.4 & 19.95697 \\
\hline$C Z_{4}$ & 0.2458594 & 93.44 & 39.16432 & 0.16362247 & 93.76 & 31.30681 & 0.07180167 & 79.92 & 20.37833 \\
\hline$C Z_{5}$ & 0.1698079 & 90.84 & 32.49987 & 0.10988402 & 92.28 & 25.91898 & 0.05880575 & 77.8 & 18.65761 \\
\hline
\end{tabular}


Table 3.5: Poisson Regression Simulation Results (New Estimators), $\mathrm{P}=4, \mathrm{n}=600$, $\rho=.85$

\begin{tabular}{|c|c|c|c|c|c|c|c|c|c|}
\hline \multirow[b]{2}{*}{$\mathrm{k}$} & \multicolumn{3}{|c|}{ Intercept $=-1$} & \multicolumn{3}{|c|}{ Intercept $=0$} & \multicolumn{3}{|c|}{ Intercept $=1$} \\
\hline & MSE & Perf. & MAPE & MSE & Perf. & MAPE & MSE & Perf. & MAPE \\
\hline$M L E$ & 0.01167838 & 0 & 8.502608 & 0.004365237 & 0 & 5.207782 & 0.001547143 & 0 & 3.124962 \\
\hline$H K$ & 0.01153794 & 65.6 & 8.453743 & 0.004341133 & 53.68 & 5.193034 & 0.00154551 & 52.76 & 3.123501 \\
\hline$H K_{2}$ & 0.01161029 & 65.96 & 8.478984 & 0.004353155 & 53.76 & 5.200267 & 0.001546264 & 52.76 & 3.12419 \\
\hline$H K B$ & 0.01142269 & 65.08 & 8.413799 & 0.004319928 & 53.28 & 5.179568 & 0.001543728 & 52.6 & 3.121959 \\
\hline$L W$ & 0.01167836 & 66.4 & 8.502601 & 0.004365187 & 53.8 & 5.207751 & 0.001547142 & 52.88 & 3.124962 \\
\hline$H S L$ & 0.01133801 & 63.96 & 8.390337 & 0.004313053 & 52.4 & 5.17182 & 0.001536315 & 51.76 & 3.115244 \\
\hline$A M$ & 0.03684882 & 40.8 & 12.899259 & 0.016292862 & 37.84 & 7.788854 & 0.008506284 & 40.08 & 4.567289 \\
\hline$G M$ & 0.01155362 & 56.72 & 8.448071 & 0.004425106 & 47.64 & 5.244466 & 0.001565284 & 49.44 & 3.141106 \\
\hline Med & 0.01128418 & 62.8 & 8.368686 & 0.004294307 & 51.48 & 5.163363 & 0.001540061 & 51.52 & 3.118865 \\
\hline$K S$ & 0.01154119 & 65.6 & 8.454838 & 0.004341533 & 53.68 & 5.193264 & 0.001545515 & 52.76 & 3.123505 \\
\hline$K S_{A}$ & 0.01147227 & 65.2 & 8.427005 & 0.004330729 & 53.12 & 5.187119 & 0.001552364 & 50.48 & 3.13403 \\
\hline$K S_{\operatorname{Max}}$ & 0.01144016 & 62.84 & 8.423687 & 0.004396916 & 51.08 & 5.232922 & 0.001720823 & 46.88 & 3.258432 \\
\hline$K S_{\text {Med }}$ & 0.01160742 & 65.96 & 8.478054 & 0.004350421 & 53.72 & 5.198647 & 0.001543709 & 52.52 & 3.122044 \\
\hline$K M_{1}$ & 0.01160453 & 66.04 & 8.47635 & 0.004345627 & 53.64 & 5.195448 & 0.001542026 & 52.44 & 3.120535 \\
\hline$K M_{2}$ & 0.01153004 & 65.6 & 8.451081 & 0.004355489 & 53.8 & 5.201719 & 0.001545196 & 52.72 & 3.123253 \\
\hline$K M_{3}$ & 0.01339461 & 55.12 & 8.904883 & 0.004580054 & 49.6 & 5.322459 & 0.001730742 & 48.96 & 3.211411 \\
\hline$K M_{4}$ & 0.01162717 & 66.16 & 8.484925 & 0.004362245 & 53.8 & 5.205928 & 0.001546538 & 52.84 & 3.124422 \\
\hline$K M_{5}$ & 0.01126371 & 63.88 & 8.358653 & 0.004305227 & 53 & 5.170349 & 0.001540311 & 52.36 & 3.118959 \\
\hline$K M_{6}$ & 0.01159795 & 65.96 & 8.474695 & 0.004360609 & 53.8 & 5.204937 & 0.001546317 & 52.76 & 3.12422 \\
\hline$K M_{7}$ & 0.01141122 & 65 & 8.409056 & 0.004325866 & 53.44 & 5.183524 & 0.001542712 & 52.52 & 3.121121 \\
\hline$K M_{8}$ & 0.01152844 & 65.6 & 8.450537 & 0.004355413 & 53.8 & 5.201674 & 0.001545193 & 52.72 & 3.123251 \\
\hline$K M_{9}$ & 0.01153903 & 65.6 & 8.4541 & 0.004347791 & 53.72 & 5.197036 & 0.001545445 & 52.72 & 3.123455 \\
\hline$K M_{10}$ & 0.01161566 & 66.08 & 8.480973 & 0.004361691 & 53.8 & 5.205598 & 0.001546498 & 52.76 & 3.124389 \\
\hline$K M_{11}$ & 0.01153611 & 65.6 & 8.453118 & 0.004351266 & 53.76 & 5.199145 & 0.001545369 & 52.72 & 3.123395 \\
\hline$K M_{12}$ & 0.01159474 & 65.92 & 8.473598 & 0.004360438 & 53.8 & 5.204832 & 0.001546309 & 52.76 & 3.124214 \\
\hline$K M_{13}$ & 0.01165043 & 66.32 & 8.493008 & 0.004364233 & 53.8 & 5.207169 & 0.001546896 & 52.88 & 3.12474 \\
\hline$K M_{14}$ & 0.01153721 & 65.6 & 8.453488 & 0.004350205 & 53.76 & 5.198501 & 0.001545397 & 52.72 & 3.123417 \\
\hline$G K$ & 0.01153793 & 65.6 & 8.453739 & 0.004341133 & 53.68 & 5.193034 & 0.00154551 & 52.76 & 3.123501 \\
\hline$H M O$ & 0.06203947 & 1.28 & 21.643385 & 0.029620923 & 0.52 & 16.546456 & 0.025305244 & 0 & 10.203905 \\
\hline$A D_{H M}$ & 0.01167836 & 66.4 & 8.502601 & 0.004365236 & 53.8 & 5.207781 & 0.001547142 & 52.88 & 3.124962 \\
\hline$A D_{M e d}$ & 0.01167833 & 66.4 & 8.50259 & 0.004365233 & 53.8 & 5.20778 & 0.001547142 & 52.88 & 3.124962 \\
\hline$A D_{G M}$ & 0.01167821 & 66.4 & 8.50255 & 0.004365225 & 53.8 & 5.207774 & 0.001547142 & 52.88 & 3.124961 \\
\hline$A D_{A M}$ & 0.01207023 & 66.16 & 8.553265 & 0.004367057 & 53.72 & 5.208822 & 0.001551946 & 52.8 & 3.126643 \\
\hline$Y_{1}$ & 0.01164386 & 65.92 & 8.491623 & 0.004359091 & 53.8 & 5.203649 & 0.001546375 & 52.84 & 3.124096 \\
\hline$Y_{2}$ & 0.01166981 & 66.36 & 8.499625 & 0.004364614 & 53.8 & 5.207393 & 0.001547105 & 52.88 & 3.12493 \\
\hline$Y_{3}$ & 0.0116619 & 66.36 & 8.496907 & 0.004363814 & 53.8 & 5.206887 & 0.001547068 & 52.88 & 3.1249 \\
\hline$Y_{4}$ & 0.01167107 & 65.16 & 8.489066 & 0.004353103 & 53.56 & 5.199508 & 0.001545955 & 52.76 & 3.123742 \\
\hline$Y_{5}$ & 0.0107892 & 59.64 & 8.187567 & 0.004250423 & 52 & 5.13548 & 0.001525166 & 48.04 & 3.102835 \\
\hline$Y_{6}$ & 0.02221462 & 19.96 & 12.222615 & 0.005468852 & 33.24 & 5.967537 & 0.00195348 & 34.32 & 3.458929 \\
\hline$Y_{7}$ & 0.01173648 & 43.92 & 8.620383 & 0.004206045 & 46.28 & 5.107267 & 0.001557028 & 42.8 & 3.134523 \\
\hline$Y_{8}$ & 0.01167372 & 66.4 & 8.500968 & 0.004364905 & 53.8 & 5.207575 & 0.00154711 & 52.88 & 3.124935 \\
\hline$Y_{9}$ & 0.01110554 & 63.24 & 8.30004 & 0.004302298 & 52.64 & 5.167192 & 0.00152762 & 50.72 & 3.107004 \\
\hline$A H$ & 0.01146101 & 65.4 & 8.42728 & 0.004348057 & 53.72 & 5.197224 & 0.001544997 & 52.72 & 3.123055 \\
\hline$F G$ & 0.01110137 & 63.08 & 8.301136 & 0.004307974 & 53.16 & 5.172177 & 0.001539115 & 52.08 & 3.117793 \\
\hline$A S$ & 0.01153793 & 65.6 & 8.453741 & 0.004341133 & 53.68 & 5.193034 & 0.00154551 & 52.76 & 3.123501 \\
\hline$A S_{\operatorname{Max}}$ & 0.05917371 & 31.12 & 16.556264 & 0.027935167 & 30.4 & 10.093997 & 0.014116382 & 34.2 & 5.726085 \\
\hline$A S_{M i n}$ & 0.01152332 & 65.52 & 8.448792 & 0.004357812 & 53.8 & 5.203143 & 0.001545007 & 52.68 & 3.123095 \\
\hline$N_{1}$ & 0.01167835 & 66.4 & 8.5026 & 0.004365236 & 53.8 & 5.207781 & 0.001547142 & 52.88 & 3.124962 \\
\hline $\mathrm{N}_{2}$ & 0.01167667 & 66.4 & 8.502024 & 0.004365055 & 53.8 & 5.20767 & 0.001547135 & 52.88 & 3.124955 \\
\hline$N_{3}$ & 0.01165942 & 66.36 & 8.496059 & 0.004362625 & 53.8 & 5.206162 & 0.001546995 & 52.88 & 3.124834 \\
\hline$N_{4}$ & 0.01167504 & 66.4 & 8.501467 & 0.004364881 & 53.8 & 5.207564 & 0.001547127 & 52.88 & 3.124949 \\
\hline$C Z_{1}$ & 0.01060478 & 57.92 & 8.121172 & 0.004256448 & 51.96 & 5.13928 & 0.001528654 & 50.72 & 3.108154 \\
\hline$C Z_{2}$ & 0.01137233 & 65.04 & 8.395642 & 0.004339028 & 53.72 & 5.191508 & 0.001541958 & 52.52 & 3.120337 \\
\hline$C Z_{3}$ & 0.01119154 & 63.76 & 8.33146 & 0.004315986 & 53.28 & 5.177007 & 0.001533876 & 51.6 & 3.112606 \\
\hline$C Z_{4}$ & 0.01132634 & 64.72 & 8.379769 & 0.004333294 & 53.6 & 5.187842 & 0.001542122 & 52.56 & 3.120499 \\
\hline$C Z_{5}$ & 0.01078788 & 59.64 & 8.186934 & 0.00424976 & 51.96 & 5.135044 & 0.001525163 & 48.04 & 3.102847 \\
\hline
\end{tabular}




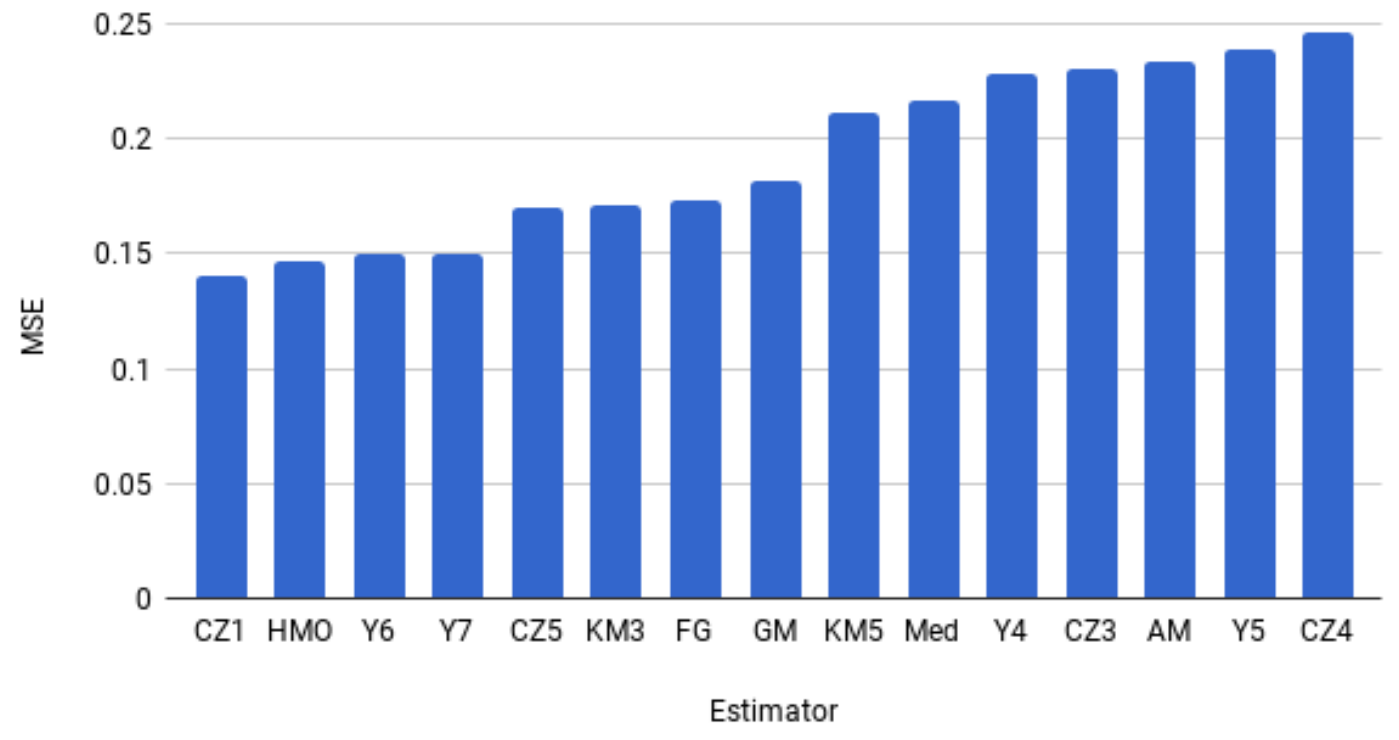

Figure 3.2: Estimators with Smallest $\operatorname{MSE}\left(\mathrm{n}=35, \rho=.85, \beta_{0}=-1\right)$ 


\section{CHAPTER 4}

\section{APPLICATION}

The ridge estimators are applied here using data from a study of 659 individuals by Sellar et al. (1985), which estimated the value of recreational boating in East Texas. Four lakes were studied: Lakes Conroe, Livingston, Somerville, and Houston. The study tracked the number of visits an individual has made to the lakes (NTrip), as well as their income level, quality rating scores for the lakes, whether the individual has engaged in waterskiing at Lake Somerville (SKI), whether the individual has paid an annual fee at Lake Somerville (FEE), and the travel costs to Lakes Conroe, Somerville, and Houston (LC, LS, and LH). The three cost variables (LC, LS, and LH) are highly correlated. The level of multicollinearity is measured here using the Variance Inflation Factor (VIF). VIF is the ratio of variance in the full model, with all terms, divided by the variance of a reduced model, with only one term. With high VIF values (VIF greater than 3), the variance of the estimated coefficient is increased. Table 4.1 shows the VIF for each regressor. It is clear from this table that LC, LS, and LH are highly correlated. In the case of severe multicollinearity, a ridge regression model is recommended. Table 4.2 shows the MSE values of the MLE model and the 55 ridge regression models. All ridge estimators had a lower MSE than the MLE model. The MLE model had an MSE of 39.61, while all of the ridge models produced an MSE smaller than 35. Most k estimators performed fairly well, with an MSE of about 31, including the five newly introduced estimators. Table 4.3 shows the significance of the regression coefficients of MLE and two of the best performing new estimators, $C Z_{1}$ and $C Z_{3}$. Table 4.4 shows the estimated regression coefficients for each model. All coefficients had a positive slope, with the exception of LS, which consistently had a negative coefficient value. This means that the number of trips (NTrip) decreases as 
LS, the travel cost to Lake Somerville, increases. The number of trips increases with an increase in the other variables (FEE, LC, and LH).

Table 4.1: VIF for each regressor

\begin{tabular}{c|c|c|c|c} 
SKI & FEE & LC & LS & LH \\
\hline 1.088497 & 1.024602 & 11.838841 & 3.188796 & 10.908486
\end{tabular}

Table 4.2: MSE for Each k Estimator - Recreation Data

\begin{tabular}{l|c|l|c|l|c|l|c}
$\mathrm{k}$ & $\mathrm{MSE}$ & $\mathrm{k}$ & $\mathrm{MSE}$ & $\mathrm{k}$ & $\mathrm{MSE}$ & $\mathrm{k}$ & $\mathrm{MSE}$ \\
\hline$M L E$ & 39.60541 & $K M_{2}$ & 31.24646 & $H M O$ & 33.11322 & $A H$ & 31.24154 \\
$H K$ & 31.23623 & $K M_{3}$ & 31.88795 & $A D_{H M}$ & 31.23278 & $F G$ & 31.34948 \\
$H K_{2}$ & 31.23444 & $K M_{4}$ & 31.23298 & $A D_{M e d}$ & 31.23278 & $A S$ & 31.23623 \\
$H K B$ & 31.26526 & $K M_{5}$ & 31.437 & $A D_{G M}$ & 31.23278 & $A S_{\text {Max }}$ & 34.99645 \\
$L W$ & 31.23278 & $K M_{6}$ & 31.2334 & $A D_{A M}$ & 31.23278 & $A S_{\text {Min }}$ & 31.25382 \\
$H S L$ & 34.21166 & $K M_{7}$ & 31.54585 & $Y_{1}$ & 31.23322 & $N_{1}$ & 31.23278 \\
$A M$ & 33.78956 & $K M_{8}$ & 31.24646 & $Y_{2}$ & 31.2328 & $N_{2}$ & 31.23278 \\
$G M$ & 32.33407 & $K M_{9}$ & 31.23828 & $Y_{3}$ & 31.23288 & $N_{3}$ & 31.2328 \\
$M e d$ & 33.32627 & $K M_{10}$ & 31.23298 & $Y_{4}$ & 31.23651 & $N_{4}$ & 31.23278 \\
$K S$ & 31.23623 & $K M_{11}$ & 31.24073 & $Y_{5}$ & 31.56081 & $C Z_{1}$ & 31.66421 \\
$K S_{A}$ & 32.66997 & $K M_{12}$ & 31.23341 & $Y_{6}$ & 33.47754 & $C Z_{2}$ & 31.27203 \\
$K S_{\text {Max }}$ & 34.24815 & $K M_{13}$ & 31.23278 & $Y_{7}$ & 32.29833 & $C Z_{3}$ & 31.32226 \\
$K S_{\text {Med }}$ & 31.63274 & $K M_{14}$ & 31.24003 & $Y_{8}$ & 31.23278 & $C Z_{4}$ & 31.29118 \\
$K M_{1}$ & 31.30317 & $G K$ & 31.23623 & $Y_{9}$ & 31.35176 & $C Z_{5}$ & 31.56081
\end{tabular}

Table 4.3: Significance of Coefficients from MLE and Selected New Estimators

\begin{tabular}{l|c|c|c|c|c|c} 
& \multicolumn{2}{|c|}{ MLE } & \multicolumn{2}{c|}{$C Z_{1}$} & \multicolumn{2}{c}{$C Z_{3}$} \\
\hline Coefficient & Z-Value & P-Value & Z-Value & P-Value & Z-Value & P-Value \\
\hline SKI & 9.737 & 0 & 16.20397 & 0 & 15.13735 & 0 \\
FEE & 14.380 & 0 & 32.1527 & 0 & 31.83864 & 0 \\
LC & 2.660 & 0.00781 & 15.3222 & 0 & 14.96995 & 0 \\
LS & -39.974 & 0 & -58.57733 & 0 & -56.72167 & 0 \\
LH & 16.499 & 0 & 23.76201 & 0 & 22.95511 & 0
\end{tabular}




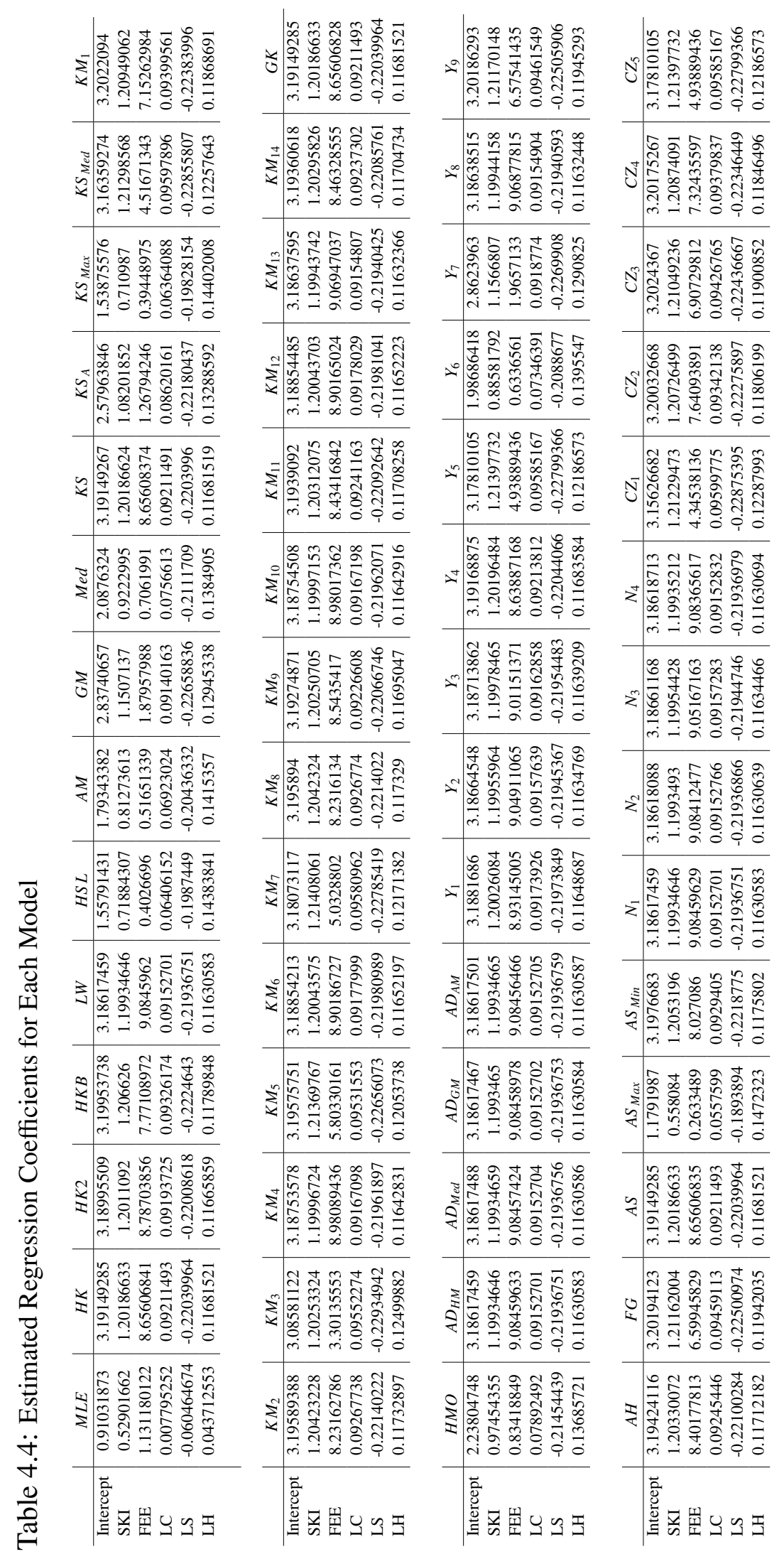




\section{CHAPTER 5}

\section{SUMMARY AND CONCLUDING REMARKS}

In this thesis, we investigated some ridge regression (RR) estimators for estimating the ridge regression parameter $\mathrm{k}$ for the Poisson regression model, when the explanatory variables are moderate to highly correlated. Since a theoretical comparison among the estimators is not possible, a Monte Carlo simulation study has been conducted to compare the performance of the proposed ridge regression estimators. In simulation, we evaluated proposed estimators of $\mathrm{k}$ for different experimental conditions: the degree of correlation, sample size, and intercept value. For each combination we performed 2500 replications. The evaluation of the performance of these estimators has been done by using the MSE, MAPE, and the proportion of times the RR estimators outperformed the ML estimator. The performance of the estimators in presence of outliers was also analyzed.

The simulation results show that increasing the correlation among the explanatory variables has a negative effect on the performance of the estimators (i.e. the MSE and MAPE increases). In most of the cases, ridge regression estimators outperform the ML estimator, even when the correlation between the explanatory variables is large. When the sample size increases, the MSE and MAPE values decreases for all estimators, including the ML estimator. From this analysis, the six best performing estimators were chosen. Based on these six best estimators, five new estimators were proposed, $C Z_{1}, C Z_{2}, C Z_{3}$, $C Z_{4}$, and $C Z_{5}$. Simulations were then run again with these five new estimators added in. These five new estimators performed very well, producing small MSE values. The best performing of these new estimators were $C Z_{1}, C Z_{3}$, and $C Z_{5}$, with $C Z_{1}$ often producing the lowest MSE. These three estimators are therefore recommended for PRR models.

Data from a study on recreational habits was used to illustrate the findings of the 
thesis. This study tracked the number of boating trips 659 individuals took to four lakes in East Texas. Among the variables studied, the cost of travel to three lakes: Conroe, Somerville, and Houston, were highly correlated, which produced severe multicollinearity with the MLE model. MSE was decreased with all 55 PRR models, so the accuracy of prediction was increased.

We have considered the Poisson regression model. As a future research, this thesis can further be extended for the following models:

i Two parameter Poisson regression model. That means, a model with both ridge regression estimator and Liu (1993) estimator.

ii Ridge Regression zero inflated Poisson regression model.

iii Ridge Regression Based on Some Robust Estimators (see Samkar and Alpu, 2010).

iv Ridge regression estimators for the restricted linear model.

v Restricted ridge regression estimators for a semiparametric regression model. 


\section{REFERENCES}

1. Adnan, K, Yasin, A. and Asir, G. "Some new modifications of Kibria's and Dorugade's methods: An application to Turkish GDP data." Journal of the Association of Arab Universities for Basic and Applied Sciences, vol. 20, 2014, pp. 89-99.

2. Al-Hassan, Yazid M. "Performance of new ridge regression estimators." Journal of the Association of Arab Universities for Basic and Applied Science, vol. 9, no. 1, 2010, pp. 23-26.

3. Alkhamisi, Mahdi, et al. Some Modifications for Choosing Ridge Parameters. Communications in Statistics - Theory and Methods, vol. 35, no. 11, 2006, pp. 2005-2020.

4. Alkhamisi, M. A., and G. Shukur. "Developing Ridge Parameters For SUR Model." Communications In Statistics - Theory And Methods, vol. 37, no. 4, 2008, pp. 544564.

5. Allen, Michael Patrick. Understanding Regression Analysis. New York, Plenum Press, 1997, p. 176.

6. Asar, Yasin, and Genç, Aşır. "A note on some new modifications of ridge estimators.” Kuwait Journal of Science, vol. 44, no. 3, 2017, pp. 75-82

7. Batah, Feras Sh. M., et al. Combining Unbiased Ridge and Principal Component Regression Estimators. Communications in Statistics - Theory and Methods, vol. 38, no. 13, 2009, pp. 2201-2209.

8. Dorugade, A.V. On Comparison of Some Ridge Parameters in Ridge Regression. Sri Lankan Journal of Applied Statistics, vol. 15 no. 1, 2014, pp. 31-46.

9. Dorugade, A.V. New Ridge Parameters for Ridge Regression. Journal of the Association of Arab Universities for Basic and Applied Sciences, vol. 15, 2013, pp. 94-99. 
10. Greene, William H. Econometric Analysis. 7th ed., Boston, Mass., Pearson, 2012.

11. Hocking, R. R. et al. "A Class Of Biased Estimators In Linear Regression." Technometrics, vol. 18, no. 4, 1976, p. 425.

12. Hoerl, Arthur E., and Robert W. Kennard. "Ridge Regression: Biased Estimation For

Nonorthogonal Problems." Technometrics, vol. 12, no. 1, 1970, pp. 55-67.

13. Hoerl, Arthur et al. "Ridge Regression: Some Simulations." Communications In Statistics - Simulation And Computation, vol. 4, no. 2, 1975, pp. 105-123.

14. Khalaf, Ghadban. A Proposed Ridge Parameter to Improve the Least Square Estimator. Journal of Modern Applied Statistical Methods, vol. 11, no. 2, Jan. 2012, pp. 443-449.

15. Khalaf, Ghadban, and Ghazi Shukur. Choosing Ridge Parameter for Regression Problems. Communications in Statistics - Theory and Methods, vol. 34, no. 5, 2005, pp. 1177-1182.

16. Kibria, B. M. Golam, and Shipra Banik. "Some Ridge Regression Estimators And Their Performances." Journal Of Modern Applied Statistical Methods, vol. 15, no. 1, 2016, pp. 206-238.

17. Kibria, B. M. Golam. "Performance Of Some New Ridge Regression Estimators." Communications In Statistics - Simulation And Computation, vol. 32, no. 2, 2003, pp. 419-435.

18. Kibria, B. M. Golam, et al. Performance of Some Logistic Ridge Regression Estimators. Computational Economics, vol. 40, no. 4, 2011, pp. 401-414.

19. Lawless, J., and P. Wang. "A Simulation Study Of Ridge And Other Regression Estimators." Communications In Statistics - Theory And Methods, vol. 5, no. 4, 1976, pp. 307-323.

20. Lee, A. H. and Silvapulle, M. J.Ridge Estimation in Logistic Regression. Communications in Statistics - Simulation and Computation,vol. 17, no. 4, 1998,pp.1231- 
21. Liu, K. "A new class of biased estimate in linear regression." Communications in Statistics - Theory and Methods, vol 22, no. 2, 1993, pp. 393-402

22. Månsson, Kristofer, and Ghazi Shukur. "A Poisson Ridge Regression Estimator." Economic Modelling, vol. 28, no. 4, 2011, pp. 1475-1481.

23. Muñiz, Gisela, and B. M. Golam Kibria. "On Some Ridge Regression Estimators: An Empirical Comparisons." Communications In Statistics - Simulation And Computation, vol. 38, no. 3, 2009, pp. 621-630.

24. Muñiz, G., Kibria, B. M. G., Månsson, K., and Shukur, G. ’On developing ridge regression parameters: A graphical investigation." Statistics and Operations Research Transactions, vol. 36, no. 2, 2012, pp. 115-138.

25. Nomura, Masuo. On the Almost Unbiased Ridge Regression Estimator. Communications in Statistics - Simulation and Computation, vol. 17, no. 3, 1988, pp. 729743.

26. Ozuna, Teofilo Jr., and Gomez, Irma Adriana. "Estimating a System of Recreation Demand Functions Using a Seemingly Unrelated Poisson Regression Approach". The Review of Economics and Statistics, vol. 76, no. 2, 1994, pp. 356-360.

27. Samkar, Hatice and Alpu, Ozlem. (2010) "Ridge Regression Based on Some Robust Estimators." Journal of Modern Applied Statistical Methods, vol. 9, no. 2, Article 17, 2010, 495-501

28. Sellar, C., Stoll, J.R., and Chavas, J.P. "Validation of Empirical Measures of Welfare Change: A Comparison of Nonmarket Techniques". Land Economics, vol. 61, (1985), pp. 156-175. 
APPENDIX

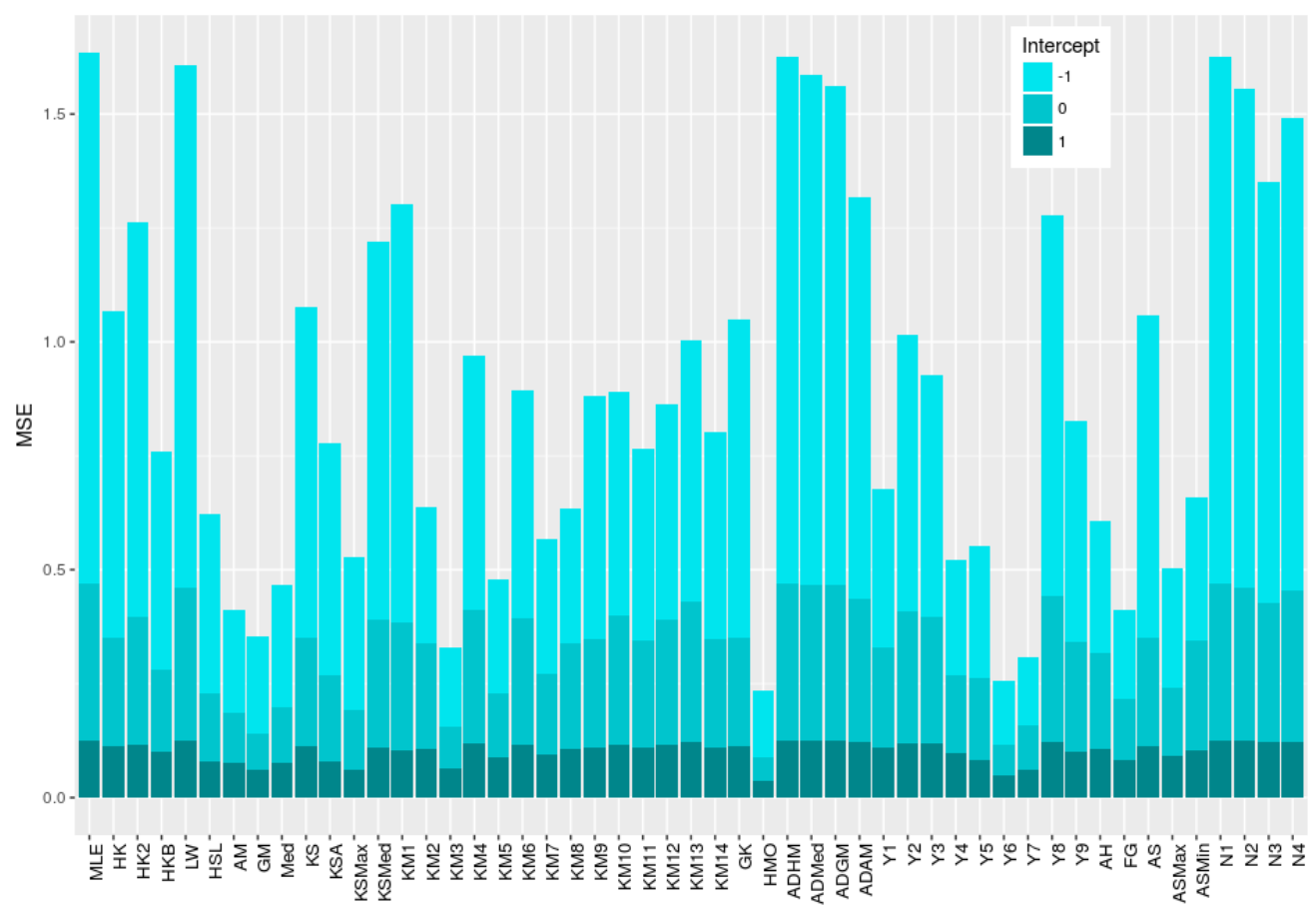

Figure A1: MSE for each k estimator, $\rho=.90$ 


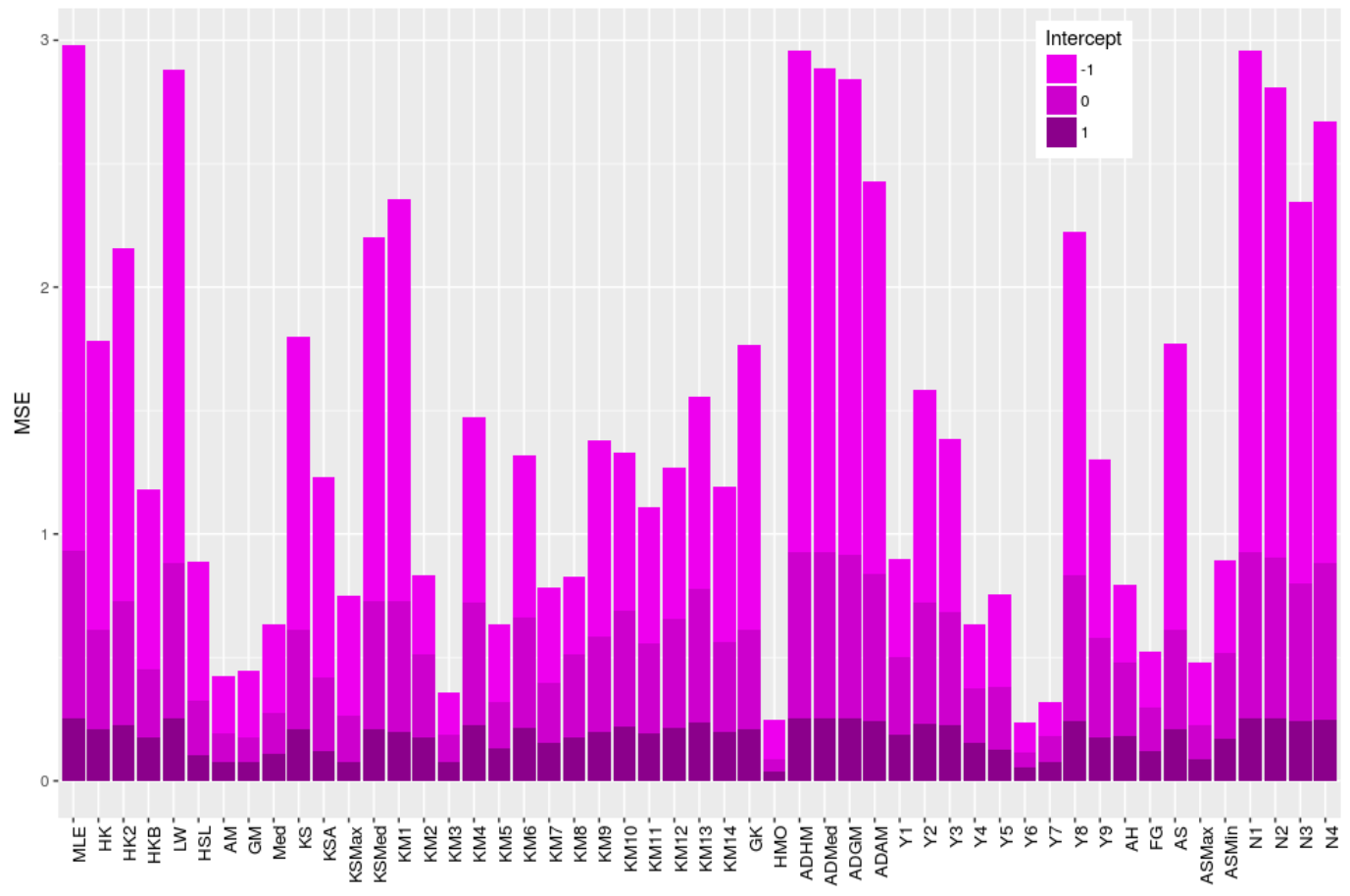

Figure A2: MSE for each k estimator, $\rho=.95$

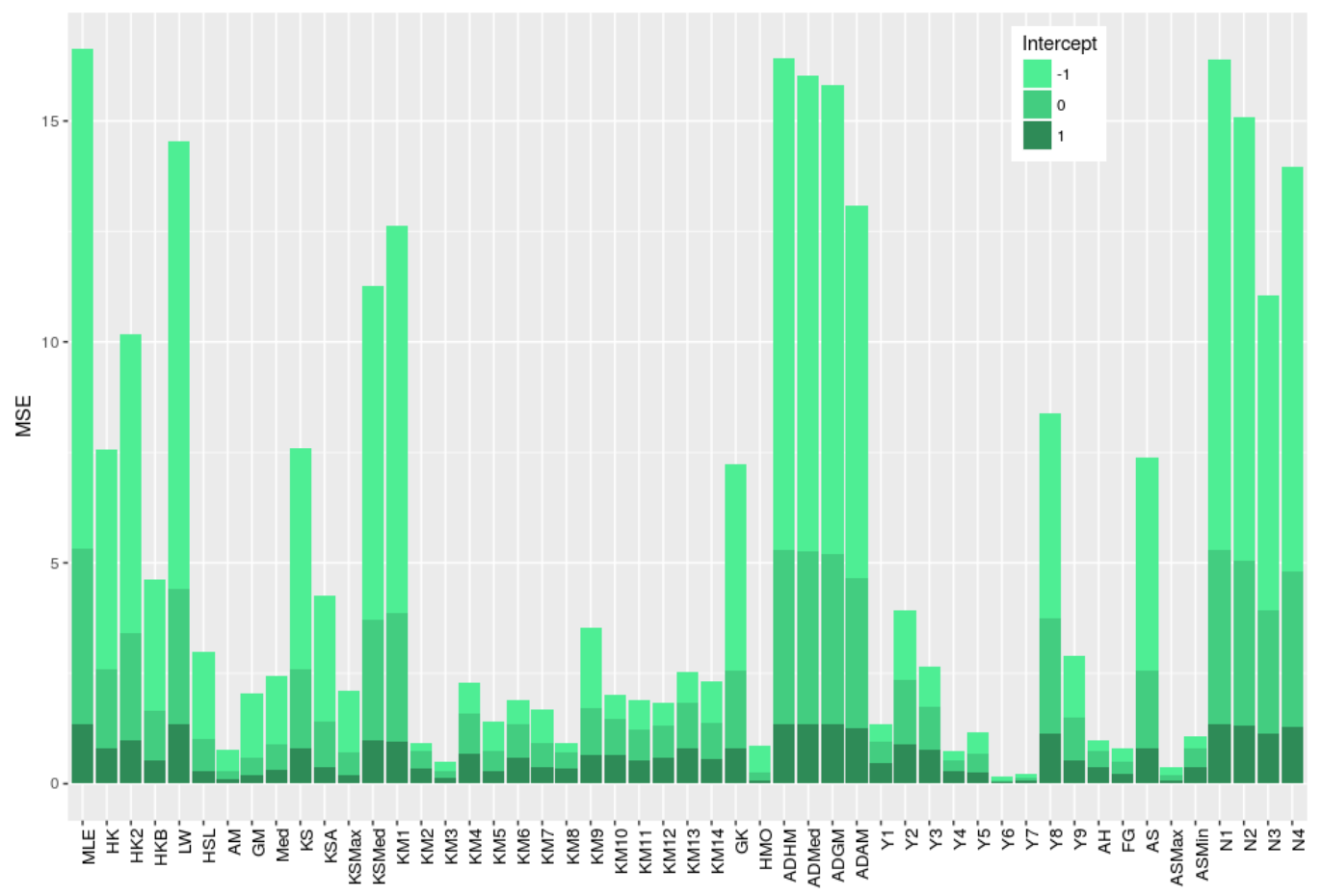

Figure A3: MSE for each k estimator, $\rho=.99$ 
Table A1: Poisson Regression Simulation Results, $\mathrm{P}=4, \mathrm{n}=35, \rho=.90$

\begin{tabular}{|c|c|c|c|c|c|c|c|c|c|}
\hline \multirow[b]{2}{*}{$\mathrm{k}$} & \multicolumn{3}{|c|}{ Intercept $=-1$} & \multicolumn{3}{|c|}{ Intercept $=0$} & \multicolumn{3}{|c|}{ Intercept $=1$} \\
\hline & MSE & Performance & MAPE & MSE & Performance & MAPE & MSE & Performance & MAPE \\
\hline$M L E$ & 1.164 & 0 & 77.745 & 0.347 & 0 & 44.192 & 0.124 & 0 & 26.685 \\
\hline$H K$ & 0.716 & 96.72 & 61.556 & 0.238 & 96.2 & 36.754 & 0.112 & 87.76 & 25.485 \\
\hline$H K_{2}$ & 0.865 & 96.96 & 67.510 & 0.280 & 96.64 & 39.886 & 0.117 & 87.84 & 26.008 \\
\hline$H K B$ & 0.478 & 95.96 & 50.944 & 0.180 & 95.88 & 32.190 & 0.101 & 87.32 & 24.329 \\
\hline$L W$ & 1.147 & 97.48 & 77.295 & 0.335 & 96.72 & 43.543 & 0.124 & 88.04 & 26.682 \\
\hline$H S L$ & 0.392 & 95.08 & 45.112 & 0.149 & 95.44 & 28.978 & 0.080 & 85.56 & 21.601 \\
\hline$A M$ & 0.226 & 85.96 & 39.106 & 0.112 & 84.04 & 25.580 & 0.075 & 77.04 & 20.003 \\
\hline$G M$ & 0.213 & 92.68 & 35.018 & 0.079 & 92.64 & 21.684 & 0.061 & 84.12 & 19.126 \\
\hline Med & 0.270 & 94.36 & 38.699 & 0.121 & 94.52 & 26.397 & 0.077 & 85.16 & 21.391 \\
\hline$K S$ & 0.724 & 96.8 & 61.944 & 0.240 & 96.32 & 36.928 & 0.112 & 87.76 & 25.495 \\
\hline$K S_{A}$ & 0.510 & 96.36 & 52.994 & 0.187 & 95.88 & 32.655 & 0.080 & 85.44 & 21.584 \\
\hline$K S_{\operatorname{Max}}$ & 0.335 & 94.96 & 42.734 & 0.131 & 95.08 & 27.229 & 0.062 & 82.56 & 19.065 \\
\hline$K S_{\text {Med }}$ & 0.830 & 97.16 & 68.239 & 0.279 & 96.6 & 40.129 & 0.110 & 87.6 & 25.242 \\
\hline$K M_{1}$ & 0.917 & 97.2 & 70.077 & 0.279 & 96.6 & 39.942 & 0.105 & 87.24 & 24.703 \\
\hline$K M_{2}$ & 0.298 & 96.8 & 43.659 & 0.232 & 96.68 & 37.713 & 0.107 & 87.64 & 25.053 \\
\hline$K M_{3}$ & 0.172 & 92.4 & 33.410 & 0.092 & 92.96 & 23.469 & 0.064 & 84 & 19.492 \\
\hline$K M_{4}$ & 0.558 & 97.16 & 58.970 & 0.294 & 96.68 & 41.656 & 0.118 & 87.92 & 26.158 \\
\hline$K M_{5}$ & 0.251 & 95.12 & 38.746 & 0.142 & 95.44 & 28.975 & 0.087 & 86.68 & 22.706 \\
\hline$K M_{6}$ & 0.499 & 96.96 & 55.749 & 0.278 & 96.72 & 40.603 & 0.116 & 87.88 & 25.941 \\
\hline$K M_{7}$ & 0.297 & 95.92 & 42.379 & 0.175 & 95.84 & 32.244 & 0.095 & 87.08 & 23.694 \\
\hline$K M_{8}$ & 0.295 & 96.8 & 43.452 & 0.231 & 96.68 & 37.640 & 0.107 & 87.64 & 25.047 \\
\hline$K M_{9}$ & 0.532 & 96.76 & 56.011 & 0.238 & 96.48 & 37.317 & 0.111 & 87.76 & 25.379 \\
\hline$K M_{10}$ & 0.489 & 97 & 55.555 & 0.284 & 96.72 & 41.078 & 0.117 & 87.88 & 26.085 \\
\hline$K M_{11}$ & 0.419 & 96.8 & 51.055 & 0.236 & 96.64 & 37.504 & 0.109 & 87.68 & 25.272 \\
\hline$K M_{12}$ & 0.474 & 96.96 & 54.496 & 0.274 & 96.72 & 40.394 & 0.116 & 87.84 & 25.922 \\
\hline$K M_{13}$ & 0.571 & 97.32 & 59.806 & 0.310 & 96.76 & 42.636 & 0.121 & 88.04 & 26.421 \\
\hline$K M_{14}$ & 0.454 & 96.8 & 52.698 & 0.237 & 96.56 & 37.451 & 0.110 & 87.68 & 25.310 \\
\hline$G K$ & 0.699 & 96.72 & 61.298 & 0.238 & 96.2 & 36.744 & 0.112 & 87.76 & 25.484 \\
\hline$H M O$ & 0.145 & 91.56 & 30.765 & 0.051 & 90.84 & 18.422 & 0.038 & 77.28 & 15.488 \\
\hline$A D_{H M}$ & 1.155 & 97.48 & 77.481 & 0.346 & 96.76 & 44.151 & 0.124 & 88.04 & 26.683 \\
\hline$A D_{M e d}$ & 1.118 & 97.44 & 76.469 & 0.344 & 96.76 & 44.061 & 0.124 & 88.04 & 26.675 \\
\hline$A D_{G M}$ & 1.095 & 97.36 & 75.729 & 0.342 & 96.76 & 43.889 & 0.124 & 88.04 & 26.663 \\
\hline$A D_{A M}$ & 0.880 & 96.48 & 69.282 & 0.316 & 96.04 & 42.222 & 0.121 & 87.72 & 26.322 \\
\hline$Y_{1}$ & 0.350 & 95.72 & 45.575 & 0.218 & 96.32 & 35.489 & 0.110 & 87.68 & 25.245 \\
\hline$Y_{2}$ & 0.607 & 96.88 & 59.546 & 0.290 & 96.68 & 40.919 & 0.120 & 87.96 & 26.334 \\
\hline$Y_{3}$ & 0.531 & 96.92 & 56.096 & 0.277 & 96.64 & 40.150 & 0.119 & 88 & 26.244 \\
\hline$Y_{4}$ & 0.255 & 94.64 & 39.383 & 0.170 & 95.36 & 31.275 & 0.098 & 87.2 & 23.934 \\
\hline$Y_{5}$ & 0.291 & 96.44 & 42.523 & 0.179 & 96.36 & 33.092 & 0.082 & 86.2 & 22.113 \\
\hline$Y_{6}$ & 0.138 & 92.2 & 30.894 & 0.067 & 93.8 & 20.048 & 0.050 & 81.12 & 17.591 \\
\hline$Y_{7}$ & 0.149 & 94.88 & 30.351 & 0.097 & 95.4 & 24.106 & 0.062 & 84.44 & 19.357 \\
\hline$Y_{8}$ & 0.837 & 97.24 & 69.071 & 0.320 & 96.72 & 42.757 & 0.122 & 88.04 & 26.492 \\
\hline$Y_{9}$ & 0.483 & 97 & 53.847 & 0.242 & 96.64 & 38.099 & 0.101 & 87.2 & 24.351 \\
\hline$A H$ & 0.289 & 96.48 & 42.299 & 0.211 & 96.56 & 35.769 & 0.106 & 87.64 & 24.954 \\
\hline$F G$ & 0.195 & 95.68 & 35.018 & 0.136 & 95.92 & 29.033 & 0.081 & 86.72 & 22.165 \\
\hline$A S$ & 0.707 & 96.72 & 61.422 & 0.238 & 96.2 & 36.749 & 0.112 & 87.76 & 25.485 \\
\hline$A S_{\operatorname{Max}}$ & 0.264 & 80.64 & 43.663 & 0.149 & 76.44 & 29.595 & 0.091 & 70.64 & 21.493 \\
\hline$A S_{\text {Min }}$ & 0.314 & 96.84 & 44.482 & 0.239 & 96.72 & 38.248 & 0.105 & 87.64 & 24.912 \\
\hline$N_{1}$ & 1.154 & 97.48 & 77.450 & 0.346 & 96.76 & 44.146 & 0.124 & 88.04 & 26.683 \\
\hline$N_{2}$ & 1.094 & 97.36 & 75.636 & 0.338 & 96.76 & 43.663 & 0.124 & 88.04 & 26.638 \\
\hline$N_{3}$ & 0.924 & 97.08 & 70.023 & 0.306 & 96.72 & 41.646 & 0.121 & 88 & 26.382 \\
\hline$N_{4}$ & 1.037 & 97.28 & 73.880 & 0.330 & 96.72 & 43.204 & 0.123 & 88.04 & 26.596 \\
\hline
\end{tabular}


Table A2: Poisson Regression Simulation Results, $\mathrm{P}=4, \mathrm{n}=35, \rho=.95$

\begin{tabular}{|c|c|c|c|c|c|c|c|c|c|}
\hline \multirow[b]{2}{*}{$\mathrm{k}$} & \multicolumn{3}{|c|}{ Intercept $=-1$} & \multicolumn{3}{|c|}{ Intercept $=0$} & \multicolumn{3}{|c|}{ Intercept $=1$} \\
\hline & MSE & Performance & MAPE & MSE & Performance & MAPE & MSE & Performance & MAPE \\
\hline$M L E$ & 2.049 & 0 & 105.552 & 0.677 & 0 & 61.743 & 0.254 & 0 & 37.326 \\
\hline$H K$ & 1.172 & 99.32 & 78.321 & 0.402 & 99.08 & 47.253 & 0.209 & 95.84 & 34.239 \\
\hline$H K_{2}$ & 1.431 & 99.36 & 87.297 & 0.502 & 99.16 & 52.974 & 0.226 & 95.88 & 35.476 \\
\hline$H K B$ & 0.729 & 98.96 & 61.697 & 0.281 & 98.96 & 39.534 & 0.173 & 95.88 & 31.409 \\
\hline$L W$ & 1.996 & 99.52 & 104.443 & 0.630 & 99.2 & 59.746 & 0.254 & 95.92 & 37.315 \\
\hline$H S L$ & 0.567 & 98.56 & 52.090 & 0.217 & 99 & 33.202 & 0.105 & 94.68 & 24.079 \\
\hline$A M$ & 0.231 & 92.68 & 38.964 & 0.116 & 92.64 & 25.054 & 0.077 & 89.2 & 20.026 \\
\hline$G M$ & 0.270 & 97.08 & 37.412 & 0.098 & 98.08 & 22.809 & 0.078 & 94.12 & 21.027 \\
\hline Med & 0.357 & 98 & 42.788 & 0.165 & 98.8 & 29.924 & 0.112 & 95.24 & 25.046 \\
\hline$K S$ & 1.183 & 99.36 & 78.762 & 0.405 & 99.08 & 47.480 & 0.209 & 95.84 & 34.259 \\
\hline$K S_{A}$ & 0.810 & 99.08 & 65.196 & 0.300 & 99 & 40.312 & 0.120 & 94.92 & 25.742 \\
\hline$K S_{\text {Max }}$ & 0.484 & 98.64 & 49.335 & 0.187 & 99 & 30.987 & 0.078 & 93.84 & 20.613 \\
\hline$K S_{\text {Med }}$ & 1.478 & 99.36 & 90.789 & 0.516 & 99.2 & 54.408 & 0.209 & 95.92 & 34.273 \\
\hline$K M_{1}$ & 1.629 & 99.36 & 94.486 & 0.527 & 99.2 & 54.715 & 0.199 & 95.92 & 33.325 \\
\hline$K M_{2}$ & 0.319 & 99.28 & 45.152 & 0.336 & 99.16 & 45.955 & 0.178 & 95.88 & 32.457 \\
\hline$K M_{3}$ & 0.176 & 96.64 & 33.264 & 0.106 & 98.08 & 24.352 & 0.078 & 94.12 & 21.283 \\
\hline $\mathrm{KM}_{4}$ & 0.752 & 99.36 & 68.660 & 0.497 & 99.2 & 54.706 & 0.223 & 95.84 & 35.658 \\
\hline$K M_{5}$ & 0.315 & 98.6 & 42.054 & 0.191 & 98.92 & 33.198 & 0.130 & 95.56 & 27.601 \\
\hline$K M_{6}$ & 0.652 & 99.4 & 63.897 & 0.451 & 99.2 & 52.336 & 0.213 & 95.8 & 35.013 \\
\hline$K M_{7}$ & 0.383 & 98.92 & 46.876 & 0.248 & 98.96 & 38.149 & 0.151 & 95.76 & 29.624 \\
\hline$K M_{8}$ & 0.316 & 99.24 & 44.946 & 0.334 & 99.16 & 45.847 & 0.178 & 95.88 & 32.445 \\
\hline$K M_{9}$ & 0.797 & 99.32 & 67.610 & 0.382 & 99.04 & 47.172 & 0.200 & 95.88 & 33.780 \\
\hline$K M_{10}$ & 0.637 & 99.36 & 63.542 & 0.471 & 99.2 & 53.461 & 0.219 & 95.84 & 35.446 \\
\hline$K M_{11}$ & 0.553 & 99.32 & 58.243 & 0.363 & 99.16 & 46.763 & 0.192 & 95.88 & 33.339 \\
\hline$K M_{12}$ & 0.615 & 99.36 & 62.207 & 0.444 & 99.2 & 51.968 & 0.212 & 95.84 & 34.965 \\
\hline$K M_{13}$ & 0.778 & 99.32 & 69.923 & 0.542 & 99.2 & 57.014 & 0.234 & 95.84 & 36.408 \\
\hline$K M_{14}$ & 0.626 & 99.32 & 61.347 & 0.369 & 99.12 & 46.915 & 0.195 & 95.88 & 33.494 \\
\hline$G K$ & 1.154 & 99.32 & 77.946 & 0.401 & 99.08 & 47.233 & 0.209 & 95.84 & 34.236 \\
\hline$H M O$ & 0.159 & 97.24 & 30.803 & 0.049 & 98.12 & 17.311 & 0.037 & 92.04 & 15.200 \\
\hline$A D_{H M}$ & 2.029 & 99.52 & 105.074 & 0.675 & 99.2 & 61.668 & 0.254 & 95.92 & 37.320 \\
\hline$A D_{M e d}$ & 1.960 & 99.52 & 103.352 & 0.671 & 99.2 & 61.475 & 0.254 & 95.92 & 37.300 \\
\hline$A D_{G M}$ & 1.924 & 99.52 & 102.170 & 0.663 & 99.2 & 61.112 & 0.253 & 95.92 & 37.267 \\
\hline$A D_{A M}$ & 1.591 & 98.88 & 91.583 & 0.598 & 98.88 & 57.623 & 0.242 & 95.88 & 36.416 \\
\hline$Y_{1}$ & 0.396 & 98.48 & 47.050 & 0.312 & 99.04 & 41.964 & 0.189 & 95.72 & 32.763 \\
\hline$Y_{2}$ & 0.860 & 99.16 & 69.620 & 0.491 & 99.16 & 53.178 & 0.232 & 95.84 & 36.003 \\
\hline$Y_{3}$ & 0.702 & 99.28 & 63.244 & 0.454 & 99.16 & 51.354 & 0.227 & 95.84 & 35.708 \\
\hline$Y_{4}$ & 0.260 & 97.72 & 38.791 & 0.219 & 98.68 & 34.616 & 0.153 & 95.52 & 29.451 \\
\hline$Y_{5}$ & 0.375 & 99.32 & 48.206 & 0.258 & 99.04 & 40.010 & 0.124 & 95.52 & 27.342 \\
\hline$Y_{6}$ & 0.123 & 96.96 & 29.398 & 0.062 & 98.6 & 18.746 & 0.053 & 94 & 17.934 \\
\hline$Y_{7}$ & 0.135 & 98.32 & 28.505 & 0.106 & 98.8 & 24.816 & 0.077 & 94.56 & 21.577 \\
\hline$Y_{8}$ & 1.388 & 99.4 & 88.611 & 0.592 & 99.16 & 58.209 & 0.243 & 95.84 & 36.661 \\
\hline$Y_{9}$ & 0.727 & 99.32 & 65.830 & 0.399 & 99.12 & 49.108 & 0.178 & 95.84 & 32.192 \\
\hline$A H$ & 0.316 & 99.16 & 43.973 & 0.298 & 99.16 & 42.855 & 0.180 & 95.84 & 32.489 \\
\hline$F G$ & 0.227 & 98.92 & 37.657 & 0.177 & 99 & 33.281 & 0.118 & 95.56 & 26.747 \\
\hline$A S$ & 1.163 & 99.32 & 78.130 & 0.402 & 99.08 & 47.243 & 0.209 & 95.84 & 34.238 \\
\hline$A S_{\operatorname{Max}}$ & 0.252 & 88.96 & 42.437 & 0.140 & 87.84 & 27.662 & 0.088 & 85.24 & 20.479 \\
\hline$A S_{\text {Min }}$ & 0.376 & 99.28 & 48.041 & 0.346 & 99.16 & 46.736 & 0.172 & 95.92 & 32.038 \\
\hline$N_{1}$ & 2.027 & 99.52 & 105.019 & 0.675 & 99.2 & 61.660 & 0.254 & 95.92 & 37.319 \\
\hline$N_{2}$ & 1.903 & 99.52 & 101.729 & 0.653 & 99.2 & 60.669 & 0.252 & 95.92 & 37.194 \\
\hline$N_{3}$ & 1.541 & 99.36 & 91.113 & 0.562 & 99.2 & 56.209 & 0.240 & 95.84 & 36.429 \\
\hline$N_{4}$ & 1.788 & 99.48 & 98.597 & 0.632 & 99.16 & 59.722 & 0.250 & 95.92 & 37.073 \\
\hline
\end{tabular}


Table A3: Poisson Regression Simulation Results, $\mathrm{P}=4, \mathrm{n}=35, \rho=.99$

\begin{tabular}{|c|c|c|c|c|c|c|c|c|c|}
\hline \multirow[b]{2}{*}{$\mathrm{k}$} & \multicolumn{3}{|c|}{ Intercept $=-1$} & \multicolumn{3}{|c|}{ Intercept $=0$} & \multicolumn{3}{|c|}{ Intercept $=1$} \\
\hline & MSE & Performance & MAPE & MSE & Performance & MAPE & MSE & Performance & MAPE \\
\hline$M L E$ & 11.291 & 0 & 243.819 & 3.972 & 0 & 149.278 & 1.354 & 0 & 86.213 \\
\hline$H K$ & 4.999 & 99.96 & 151.743 & 1.785 & 100 & 94.378 & 0.794 & 99.92 & 65.840 \\
\hline$H K_{2}$ & 6.761 & 99.96 & 179.106 & 2.438 & 100 & 112.498 & 0.984 & 99.96 & 73.217 \\
\hline$H K B$ & 2.978 & 99.96 & 112.984 & 1.108 & 100 & 72.709 & 0.542 & 99.88 & 53.999 \\
\hline$L W$ & 10.132 & 99.96 & 233.055 & 3.072 & 100 & 130.412 & 1.349 & 99.96 & 86.100 \\
\hline$H S L$ & 1.986 & 99.96 & 85.865 & 0.731 & 100 & 53.366 & 0.271 & 99.8 & 33.627 \\
\hline$A M$ & 0.491 & 99.2 & 45.323 & 0.174 & 98.84 & 27.074 & 0.101 & 98.16 & 20.857 \\
\hline$G M$ & 1.458 & 99.8 & 66.848 & 0.404 & 99.96 & 38.287 & 0.190 & 99.76 & 28.925 \\
\hline Med & 1.532 & 99.8 & 74.990 & 0.589 & 100 & 49.650 & 0.310 & 99.8 & 38.097 \\
\hline$K S$ & 5.018 & 99.96 & 152.225 & 1.792 & 100 & 94.664 & 0.796 & 99.92 & 65.902 \\
\hline$K S_{A}$ & 2.827 & 99.96 & 111.730 & 1.034 & 100 & 69.362 & 0.385 & 99.84 & 42.161 \\
\hline$K S_{\operatorname{Max}}$ & 1.372 & 99.96 & 74.557 & 0.521 & 100 & 46.202 & 0.198 & 99.76 & 28.773 \\
\hline$K S_{\text {Med }}$ & 7.552 & 99.96 & 202.297 & 2.732 & 100 & 125.047 & 0.983 & 99.96 & 74.327 \\
\hline$K M_{1}$ & 8.748 & 99.96 & 214.289 & 2.907 & 100 & 127.578 & 0.967 & 99.96 & 72.794 \\
\hline$K M_{2}$ & 0.187 & 99.96 & 33.587 & 0.366 & 100 & 49.325 & 0.359 & 99.92 & 47.803 \\
\hline$K M_{3}$ & 0.225 & 99.8 & 34.097 & 0.149 & 99.96 & 26.772 & 0.122 & 99.8 & 24.647 \\
\hline $\mathrm{KM}_{4}$ & 0.689 & 99.96 & 64.736 & 0.917 & 100 & 77.520 & 0.681 & 99.96 & 64.905 \\
\hline$K M_{5}$ & 0.670 & 99.96 & 56.245 & 0.429 & 100 & 47.178 & 0.296 & 99.84 & 40.242 \\
\hline$K M_{6}$ & 0.549 & 99.96 & 57.661 & 0.752 & 100 & 70.107 & 0.591 & 99.96 & 60.787 \\
\hline$K M_{7}$ & 0.748 & 99.96 & 61.662 & 0.545 & 100 & 54.482 & 0.377 & 99.88 & 45.686 \\
\hline$K M_{8}$ & 0.186 & 99.96 & 33.483 & 0.365 & 100 & 49.200 & 0.358 & 99.92 & 47.768 \\
\hline$K M_{9}$ & 1.796 & 99.96 & 98.868 & 1.085 & 100 & 78.102 & 0.635 & 99.92 & 60.393 \\
\hline$K M_{10}$ & 0.539 & 99.96 & 57.701 & 0.820 & 100 & 73.589 & 0.653 & 99.96 & 63.681 \\
\hline$K M_{11}$ & 0.694 & 99.96 & 65.112 & 0.697 & 100 & 65.715 & 0.516 & 99.92 & 55.720 \\
\hline$K M_{12}$ & 0.516 & 99.96 & 56.060 & 0.736 & 100 & 69.347 & 0.587 & 99.96 & 60.584 \\
\hline$K M_{13}$ & 0.678 & 99.96 & 62.863 & 1.057 & 100 & 83.136 & 0.790 & 99.96 & 69.838 \\
\hline$K M_{14}$ & 0.952 & 99.96 & 75.185 & 0.808 & 100 & 69.697 & 0.555 & 99.92 & 57.310 \\
\hline$G K$ & 4.678 & 99.96 & 148.633 & 1.773 & 100 & 94.155 & 0.794 & 99.92 & 65.818 \\
\hline$H M O$ & 0.596 & 99.88 & 48.750 & 0.186 & 100 & 28.124 & 0.082 & 99.8 & 20.566 \\
\hline$A D_{H M}$ & 11.119 & 99.96 & 241.818 & 3.951 & 100 & 148.860 & 1.352 & 99.96 & 86.167 \\
\hline$A D_{M e d}$ & 10.772 & 99.96 & 236.858 & 3.908 & 100 & 147.970 & 1.348 & 99.96 & 86.030 \\
\hline$A D_{G M}$ & 10.610 & 99.96 & 233.916 & 3.866 & 100 & 146.918 & 1.344 & 99.96 & 85.870 \\
\hline$A D_{A M}$ & 8.420 & 99.92 & 200.200 & 3.387 & 99.96 & 135.163 & 1.263 & 99.92 & 82.544 \\
\hline$Y_{1}$ & 0.388 & 99.92 & 43.789 & 0.491 & 100 & 50.151 & 0.466 & 99.88 & 50.109 \\
\hline$Y_{2}$ & 1.581 & 99.96 & 88.678 & 1.446 & 100 & 88.867 & 0.902 & 99.96 & 70.847 \\
\hline$Y_{3}$ & 0.911 & 99.96 & 67.369 & 0.975 & 100 & 73.775 & 0.764 & 99.96 & 66.127 \\
\hline$Y_{4}$ & 0.208 & 99.76 & 33.814 & 0.251 & 100 & 34.663 & 0.281 & 99.8 & 37.784 \\
\hline$Y_{5}$ & 0.474 & 99.96 & 53.107 & 0.426 & 100 & 52.373 & 0.261 & 99.88 & 40.030 \\
\hline$Y_{6}$ & 0.102 & 99.84 & 28.608 & 0.031 & 100 & 12.833 & 0.033 & 99.8 & 13.683 \\
\hline$Y_{7}$ & 0.083 & 99.96 & 23.477 & 0.066 & 100 & 18.568 & 0.070 & 99.8 & 20.074 \\
\hline$Y_{8}$ & 4.627 & 99.96 & 158.168 & 2.613 & 100 & 121.342 & 1.143 & 99.96 & 79.579 \\
\hline$Y_{9}$ & 1.413 & 99.96 & 87.511 & 0.963 & 100 & 76.483 & 0.526 & 99.96 & 56.370 \\
\hline$A H$ & 0.226 & 99.96 & 36.227 & 0.361 & 100 & 47.093 & 0.381 & 99.92 & 48.771 \\
\hline$F G$ & 0.313 & 99.96 & 43.910 & 0.280 & 100 & 42.022 & 0.216 & 99.84 & 36.616 \\
\hline$A S$ & 4.820 & 99.96 & 150.075 & 1.779 & 100 & 94.266 & 0.794 & 99.92 & 65.829 \\
\hline$A S_{\operatorname{Max}}$ & 0.190 & 98.92 & 36.273 & 0.104 & 97.88 & 22.550 & 0.075 & 97 & 18.056 \\
\hline$A S_{\text {Min }}$ & 0.294 & 99.96 & 40.382 & 0.427 & 100 & 52.359 & 0.366 & 99.92 & 48.214 \\
\hline$N_{1}$ & 11.100 & 99.96 & 241.594 & 3.949 & 100 & 148.811 & 1.352 & 99.96 & 86.162 \\
\hline$N_{2}$ & 10.057 & 99.96 & 228.038 & 3.713 & 100 & 143.696 & 1.320 & 99.96 & 85.159 \\
\hline$N_{3}$ & 7.125 & 99.96 & 186.178 & 2.794 & 100 & 122.003 & 1.128 & 99.96 & 78.665 \\
\hline$N_{4}$ & 9.172 & 99.96 & 216.316 & 3.507 & 100 & 139.134 & 1.289 & 99.96 & 84.198 \\
\hline
\end{tabular}


Table A4: Poisson Regression Simulation Results, $\mathrm{P}=4, \mathrm{n}=600, \rho=.90$

\begin{tabular}{|c|c|c|c|c|c|c|c|c|c|}
\hline \multirow[b]{2}{*}{$\mathrm{k}$} & \multicolumn{3}{|c|}{ Intercept $=-1$} & \multicolumn{3}{|c|}{ Intercept $=0$} & \multicolumn{3}{|c|}{ Intercept $=1$} \\
\hline & MSE & Perf. & MAPE & MSE & Perf. & MAPE & & Perf. & MAPE \\
\hline$M L E$ & 0.01644419 & 0 & 10.113587 & 0.006143805 & 0 & 6.180165 & 0.002307547 & 0 & 3.777529 \\
\hline$H K$ & .01618726 & 9.04 & 10.033184 & .006079381 & & 149478 & .002303666 & & 3.774601 \\
\hline$H K_{2}$ & 1631803 & 9.32 & 10.074814 & .006110535 & 58.28 & 164242 & .002305364 & & 3.77588 \\
\hline & 01596544 & 68.64 & 9.965104 & .006016526 & 57.88 & & .002298984 & & 3.771046 \\
\hline$L W$ & 01644415 & 69.72 & 10.113576 & .006143631 & & & .002307547 & 53.8 & 3.777529 \\
\hline & 1564847 & 67.28 & 9.875409 & .005911837 & 56.48 & & 002277866 & & 3.755612 \\
\hline & 03625134 & 46.28 & 13.568661 & 5410892 & 44.52 & & 07942872 & & 4.929 \\
\hline$G M$ & 0153435 & 62.08 & 9.782934 & 5773678 & 53.04 & .012892 & 02284786 & & 7576 \\
\hline Med & 01551666 & 66.6 & 9.834 & 5816435 & & 16 & 02279947 & 52.2 & $3.75^{\circ}$ \\
\hline & 1619243 & 69.04 & 10.0347 & .006080327 & & & 02303676 & & 3.77 \\
\hline$K S_{A}$ & 1602815 & 68.64 & 9.990 & 986324 & 57.28 & 45 & 290224 & & 3.76 \\
\hline$K S_{\text {Max }}$ & 01575215 & 66 & 9.908468 & .00590323 & & & 02514652 & & \\
\hline$K S_{\text {Med }}$ & & 69.36 & 10 & & & & 2299872 & & \\
\hline$K M_{1}$ & 1632989 & 69.32 & 0.078 & .006096174 & & 35 & 2295128 & & 3.7 \\
\hline & 1617109 & 69.08 & 10.030 & .006117343 & & & 230267 & & 3.77 \\
\hline & & 0.8 & & & & & & & \\
\hline & 4996 & 69.5 & 10.08 & 3544 & & & 06164 & & 3.77 \\
\hline & 6312 & & & & & & & & \\
\hline & & 6 & & & & & & & 3.77 \\
\hline & 1148 & 6 & & 4205 & & & & & 3.76 \\
\hline & & 69.08 & & & & & 302664 & & \\
\hline & & 69 & & & & & & & \\
\hline & 3094 & 69.4 & & & & & 306066 & & 3.77 \\
\hline & 8207 & 9.0 & & 6117 & & & 303194 & & 3.77 \\
\hline & 9756 & 69.4 & & & & & & & 3.77 \\
\hline & & & & & & & & & \\
\hline & & 69 & & & & & & & 3.77 \\
\hline & 3724 & 69.04 & 79 & 7938 & 58. & & & & 3.77 \\
\hline$M O$ & & & & & & & & & \\
\hline$D_{H M}$ & & 6 & & & & & & & \\
\hline D.t. & & & & & & & & & 3.77 \\
\hline$D_{G M}$ & 392 & 69.7 & 10.1 & 771 & & & 546 & & 3.77 \\
\hline$D_{A M}$ & & & & & & & & & \\
\hline & & & & & & & 306634 & & 3.77 \\
\hline$Y$ & 2679 & 69.6 & 10.1 & 2011 & & & 230745 & & 3.77 \\
\hline & & & & & & & & & \\
\hline & & & & & & & & & \\
\hline & 4017 & 64.8 & & 3047 & & & 30718 & & 3.71 \\
\hline & & 30.0 & 12.479 & & & & & & 4.093206 \\
\hline$Y_{7}$ & & & & & & & & & 3.724 \\
\hline & & & & & & & & & 3.777465 \\
\hline & & & & & & & & & 3.741648 \\
\hline$A H$ & & & & & & & & & \\
\hline & & & & & & & 0.002287307 & & 3.762136 \\
\hline & & & & & & & 303666 & & 3.774601 \\
\hline$A S_{\operatorname{Max}}$ & & 36.56 & & & & & 0.013369946 & & 5.967883 \\
\hline & 1616012 & 69.12 & 10.027718 & & & & 0.002302119 & 53.52 & 3.773422 \\
\hline$N_{1}$ & & & & & & & & 53.8 & 3.777529 \\
\hline$N_{2}$ & 0.01644124 & 69.72 & 10.112698 & 0.006143336 & 58.48 & 6.179943 & 0.002307528 & 53.8 & 3.777516 \\
\hline & .01640858 & 69.6 & 10.102734 & 0.006136516 & 58.4 & 6.17669 & 0.002307178 & 53.8 & 3.777252 \\
\hline$N_{4}$ & 0.01643839 & 69.72 & 10.111837 & 0.006142884 & 58.48 & 6.179728 & 0.002307511 & 53.8 & 3.777503 \\
\hline
\end{tabular}


Table A5: Poisson Regression Simulation Results, $\mathrm{P}=4, \mathrm{n}=600, \rho=.95$

\begin{tabular}{|c|c|c|c|c|c|c|c|c|c|}
\hline \multirow[b]{2}{*}{$\mathrm{k}$} & \multicolumn{3}{|c|}{ Intercept $=-1$} & \multicolumn{3}{|c|}{ Intercept $=0$} & \multicolumn{3}{|c|}{ Intercept $=1$} \\
\hline & MSE & Perf. & MAPE & MSE & Perf. & MAPE & MSE & Perf. & MAPE \\
\hline$M L E$ & 0.03458358 & 0 & 14.55558 & 0.012413174 & 0 & 8.726677 & 0.004580848 & 0 & 5.308665 \\
\hline$H K$ & 0.03344277 & 84.4 & 14.31904 & 0.012127558 & 71.4 & 8.633023 & 0.004564648 & 62.12 & 5.299951 \\
\hline$H K_{2}$ & 0.03403635 & 84.56 & 14.44451 & 0.012279257 & 71.48 & 8.682858 & 0.004571297 & 62.12 & 5.303527 \\
\hline$H K B$ & 0.03251126 & 84.08 & 14.12995 & 0.011902401 & 71.12 & 8.558453 & 0.004543209 & 61.96 & 5.288303 \\
\hline$L W$ & 03458342 & 84.68 & 14.55555 & 0.012412145 & 71.56 & 8.726348 & .004580848 & 62.16 & 5.308664 \\
\hline$H S L$ & 03158292 & 83.48 & 13.92679 & 0.011619709 & 70.68 & 8.459533 & .004397704 & 60.88 & 5.208903 \\
\hline$A M$ & 0.03841106 & 66.04 & 14.89378 & 0.015934679 & 62.6 & 9.023931 & .007218134 & 51.2 & 5.681794 \\
\hline$G M$ & 02670301 & 80.12 & 12.87794 & 0.01051479 & 68.24 & 8.094192 & 0.004362637 & 60.08 & 5.181228 \\
\hline Med & 0.03063955 & 83.44 & 13.74432 & 0.011341153 & 70.52 & 8.367328 & 0.004433912 & 60.88 & 5.228096 \\
\hline$K S$ & 03346558 & 84.4 & 14.32379 & 0.012132006 & & 8.634448 & .004564686 & & \\
\hline$K S_{A}$ & 03330681 & 84.32 & 14.28787 & 0.012033281 & 71.2 & 8.60 & 4457497 & 60.96 & 5.23 \\
\hline$K S_{\operatorname{Max}}$ & 0.03150779 & 83.32 & 13.91903 & 0.01155199 & 70.48 & 8.440359 & 445313 & 58.52 & 5.222302 \\
\hline$K S_{\text {Med }}$ & 3426793 & 54 & $14.4 C$ & 0.01231925 & & & 556832 & & 5.29 \\
\hline$K M_{1}$ & 0.03429089 & 84.64 & 14.49534 & 0.012301508 & 71.48 & $8.689^{\circ}$ & 4543692 & .88 & 5.288 \\
\hline$K M_{2}$ & 0.03342205 & 84.32 & 14.32359 & 0.012312141 & 71.52 & 8.693 & 559416 & 62.12 & 713 \\
\hline$K M_{3}$ & 02776817 & 79.48 & 13. & 165324 & 69. & 8.34 & 435591 & 59.8 & 5.20 \\
\hline$K M_{4}$ & 0.03417513 & 84.56 & 14.47428 & 0.012377034 & 71.52 & 8.7 & 4574996 & 62.12 & 5.30 \\
\hline$K M_{5}$ & 3116501 & 54 & 13.8 & 329545 & 71 & & 09034 & & \\
\hline$K M_{6}$ & 03393166 & 84.52 & 14.42 & 360586 & 71.52 & 8.70 & 573457 & & 5.30 \\
\hline$K M_{7}$ & 03245914 & 84.04 & 14.12245 & 18559 & 71. & 8.59 & 135 & 61.8 & 5.278 \\
\hline$K M_{8}$ & 41104 & 84.32 & 14.3 & 11407 & 7 & 8.69 & 559392 & & 5.29 \\
\hline$K M_{9}$ & 03346212 & 84.4 & 14.3 & 215379 & 71. & 8.66 & 563289 & .12 & 5.29 \\
\hline$K M_{10}$ & 03409362 & 84.56 & 14.4 & 73017 & 7 & & 574624 & 62.12 & 531 \\
\hline$M_{11}$ & 5004 & 4.36 & 14.3 & 60031 & 7 & 8.67 & 456202 & & 5.29 \\
\hline$K M_{12}$ & 03390997 & 84.52 & 14.4 & 359105 & 71.5 & 8.70 & 457338 & & 5.30 \\
\hline$K M_{13}$ & 0.03436751 & 84.64 & 14.51297 & 0.01240143 & 71.52 & 8.72 & 578722 & & 5.30 \\
\hline$K M_{14}$ & 03345498 & 84.36 & 14.3 & 246537 & 71.48 & 8.6 & 562466 & & 5.29 \\
\hline$G K$ & 03344269 & 84.4 & 14.31 & .012127554 & 71.4 & 8.63 & 564648 & 62.12 & 5.25 \\
\hline$M O$ & 03102247 & 43.64 & 14.8 & 302409 & & 10.54 & 776803 & & \\
\hline$A D_{H M}$ & 8343 & & & 161 & & & 348 & & 5.3 \\
\hline$A D_{M e d}$ & 3458323 & & & & & & 80847 & & 563 \\
\hline$A D_{G M}$ & .0345824 & 84.68 & 14.5 & 413088 & 71. & 8.72 & 580845 & & 5.30 \\
\hline$A D_{A M}$ & 03453621 & & & & & & 576 & & \\
\hline & 0.03388606 & & 14.41153 & 0.012346257 & & 8.705 & 4575746 & & 5.306206 \\
\hline$Y_{2}$ & 0.03448593 & 84.68 & 14.536 & 0.012404888 & 71.5 & 2402 & 580321 & 62.16 & 5.308 \\
\hline$Y_{3}$ & 3438291 & 84 & 14.5 & 283 & & & 832 & & 5.30 \\
\hline$Y_{4}$ & 0.03307935 & 83.96 & 14.25003 & 0.012265708 & 71. & 8.68 & 0.004566766 & 62.12 & 5.30 \\
\hline$Y_{5}$ & 0.02856843 & 82.6 & 13.26743 & 0.011394106 & 70.56 & 8.38826 & 0.004250934 & & 5.122194 \\
\hline$Y_{6}$ & 02423757 & 56.36 & 12.79749 & 0.008663162 & 57.28 & 7.568889 & 0.004352929 & 45 & 5.159493 \\
\hline$Y_{7}$ & 0.02020237 & 73.16 & 11.27589 & 0.009142933 & 66.12 & 7.556878 & 4002908 & 54.72 & 4.974802 \\
\hline$Y_{8}$ & 0.03454707 & 84.68 & 14.54839 & 0.012409395 & 71.52 & 8.725471 & 0.004580446 & 62.16 & 5.308451 \\
\hline$Y_{9}$ & 0.03115142 & 83.72 & & 0.011831967 & 71 & 8.531566 & 0.00442437 & 61.16 & 5.220933 \\
\hline$A H$ & 0.03287238 & 84.24 & 14.20922 & 0.012235144 & 71.48 & 8.668876 & 0.00455907 & 62.12 & 5.296945 \\
\hline$F G$ & 0.03000344 & 83.4 & 13.60061 & 0.011788187 & 71.08 & 8.521492 & 0.004492202 & 61.68 & 5.26027 \\
\hline$A S$ & 0.03344273 & 84.4 & 14.31903 & 0.012127556 & 71.4 & 8.633022 & 0.004564648 & & 5.29995 \\
\hline$A S_{\operatorname{Max}}$ & 0.05193563 & 56.68 & 16.93155 & 0.021052485 & 57.36 & 9.800755 & 0.010651341 & 44.24 & 6.242862 \\
\hline$A S_{\text {Min }}$ & 0.033397 & 84.32 & 14.32126 & 0.012339646 & 71.52 & 8.702994 & 0.004556814 & 62.08 & 5.295721 \\
\hline$N_{1}$ & 0.03458341 & 84.68 & 14.55555 & 0.012413159 & 71.56 & 8.726672 & 0.004580848 & 62.16 & 5.308664 \\
\hline$N_{2}$ & 0.03457087 & 84.68 & 14.55306 & 0.012411315 & 71.52 & 8.72608 & 0.004580769 & 62.16 & 5.308622 \\
\hline$N_{3}$ & 0.03441921 & 84.68 & 14.52254 & 0.012382018 & 71.52 & 8.716568 & 0.00457913 & 62.16 & 5.307747 \\
\hline & 0.03455844 & 84.68 & 14.5506 & 0.012409496 & 71.52 & 8.725495 & 0.004580691 & 62.16 & 5.308581 \\
\hline
\end{tabular}


Table A6: Poisson Regression Simulation Results, $\mathrm{P}=4, \mathrm{n}=600, \rho=.99$

\begin{tabular}{|c|c|c|c|c|c|c|c|c|c|}
\hline \multirow[b]{2}{*}{$\mathrm{k}$} & \multicolumn{3}{|c|}{ Intercept $=-1$} & \multicolumn{3}{|c|}{ Intercept $=0$} & \multicolumn{3}{|c|}{ Intercept $=1$} \\
\hline & MSE & Perf. & MAPE & MSE & Perf. & MAPE & MSE & Perf. & MAPE \\
\hline$M L E$ & 0.18582777 & 0 & 33.38409 & 0.06934168 & 0 & 20.485105 & 0.02582587 & 0 & 12.462976 \\
\hline$H K$ & 0.16103696 & 99.28 & 31.08224 & 0.06101044 & 8.92 & 19.262953 & 0.025309389 & 95.28 & 12.347252 \\
\hline$H K_{2}$ & 17218731 & 99.28 & 32.18449 & .06540785 & 98.92 & 19.92372 & 0.025516004 & 95.28 & 12.393898 \\
\hline$H K B$ & 14076035 & 99.28 & 29.19586 & 0.05584294 & 98.92 & 18.472268 & 0.024630175 & 95.24 & 12.193512 \\
\hline$L W$ & 18582269 & 99.32 & 33.38368 & .06927383 & 98.88 & 20.476116 & .025825857 & 95.4 & 12.462973 \\
\hline$H S L$ & 0.1251882 & 99.24 & 27.65938 & 0.05173606 & 98.96 & 17.883959 & .019922343 & 95.16 & 11.029444 \\
\hline$A M$ & 0.06746711 & 94.04 & 19.74635 & 0.02898679 & 97.44 & 12.692454 & .015968824 & 92.28 & 5563 \\
\hline GM & 07352575 & 98.84 & 20.7999 & 0.03242938 & 98.6 & 14.099061 & 8417736 & 94.88 & 10.65 \\
\hline Med & 0.10341449 & 99.24 & 25.46218 & 0.04851616 & 98.92 & 17.209502 & 1116842 & 95.12 & 11.3 \\
\hline$K S$ & 6141992 & 99.28 & 31.12135 & 06112187 & 98.92 & 19.280408 & 5310645 & 95.28 & 12.34 \\
\hline$K S_{A}$ & 15987992 & 99.28 & 31.200 & 214022 & 98.92 & 19.50 & 3067501 & 95.2 & 11.8 \\
\hline$K S_{\operatorname{Max}}$ & 0.12634891 & 99.24 & 27.80045 & 0.05192521 & 98.96 & 17.91604 & 076399 & 95.12 & 10.8 \\
\hline$K S_{\text {Med }}$ & 18376568 & 99. & 33.2 & & & 20.38 & 5599719 & 95.32 & \\
\hline$K M_{1}$ & 0.18330701 & 99.32 & 33.17 & 325879 & 98.88 & 20.33 & 5402886 & 95.28 & 12.36 \\
\hline$K M_{2}$ & 1521237 & 99.28 & 30.5 & 598494 & 98.92 & 20.03 & 5118562 & 95.28 & 12.3 \\
\hline$K M_{3}$ & 9866 & 98.5 & 21.8 & 57523 & 98. & & 384524 & 94. & 10.6 \\
\hline$K M_{4}$ & 0.17339477 & 99.28 & 32.3 & 12812 & 98.88 & 20.31 & 650753 & 95.32 & 12.4 \\
\hline$K M_{5}$ & 9492 & 99. & 26.8 & 19711 & & & 443623 & 16 & \\
\hline$K M_{6}$ & 09797 & 99.28 & 31.9 & 73468 & 98.88 & 15 & 584012 & 95.32 & 12.4 \\
\hline$K M_{7}$ & 4506 & 99.28 & 28.6 & 206 & 98.92 & & 5474 & 95.2 & 12.0 \\
\hline$M_{8}$ & 39849 & 99. & 30.5 & 6174 & 98. & 20.02 & 511776 & 95. & 12.3 \\
\hline$K M_{9}$ & 96305 & 99.2 & 30.9 & & 98.92 & 19.61 & 258807 & 95.28 & 12.3 \\
\hline$M_{10}$ & 32732 & 99.2 & 32.1 & 96037 & 8.8 & 20.2 & 632265 & 95.32 & 12.4 \\
\hline$M_{11}$ & 66941 & 99.2 & 30.8 & 47017 & 98. & 19.80 & 212343 & 95. & 12.3 \\
\hline$M_{12}$ & 42528 & 99.2 & 31.8 & 30336 & 98. & 20.21 & 581273 & 95.32 & 12.4 \\
\hline$K M_{13}$ & 0.17881039 & 99.32 & 32.81 & 392091 & 98.88 & 20.4 & 5764736 & 95.36 & 12.4 \\
\hline$M_{14}$ & 7976 & 99. & 30. & & 98.92 & 45 & 228616 & 95. & 12.32 \\
\hline & 16103502 & 99.28 & 31.0 & 01034 & 98.92 & 19.26 & 309383 & 95.28 & 12.34 \\
\hline$M O$ & 43056 & 97.52 & 11.4 & 354 & 98.0 & 6.67 & 439857 & & 576 \\
\hline$D_{H M}$ & 359 & 99. & & & 98 & & & 95.4 & 12.4 \\
\hline$D_{M e d}$ & 1178 & 99. & & & & & 325805 & 95. & 12.4 \\
\hline$A D_{G M}$ & 78998 & 99.3 & 33.3 & 33866 & 98.8 & 20.48 & 825738 & 95.4 & 12.46 \\
\hline$A D_{A M}$ & 2233 & & & & & & & 95.4 & \\
\hline & 5845314 & & & 0.06531888 & 98. & 19.948544 & 5254713 & 95.28 & 12.3 \\
\hline$Y_{2}$ & 0.18067006 & 99.32 & 32.9 & 82375 & 98.88 & 20.415953 & 5790487 & 95.4 & 12.45 \\
\hline$Y_{3}$ & & 99. & & & & & & 95.36 & 12.4 \\
\hline & 13826624 & 98.96 & 28.7 & 55693 & 98.92 & 19.26 & 410606 & 95.28 & 12.15 \\
\hline$Y_{5}$ & 11324397 & 99.24 & 26.40875 & 36886 & 98.92 & 17.998914 & 945996 & 95.04 & 11.046408 \\
\hline$Y_{6}$ & 0.01704792 & 96.04 & 10.57663 & 0.00770451 & 98.2 & 6.954882 & 7688465 & 92.04 & 6.870258 \\
\hline$Y_{7}$ & 0.02553499 & 98.16 & 12.25815 & 0.01845972 & 98. & 10.589707 & 2484885 & 94.16 & 2412 \\
\hline$Y_{8}$ & 0.18464944 & 99.32 & 33.29048 & 921206 & 98.88 & 20.467955 & 0.025810482 & 95.4 & 12.459637 \\
\hline & 0.1408882 & 99.28 & & .06100411 & & 19.257476 & 0.023811955 & 95.2 & 11.985711 \\
\hline$A H$ & 0.14285014 & 99.28 & 29.57246 & 67471 & 98.92 & 19.700715 & 0.025124756 & 95.28 & 12.307368 \\
\hline$F G$ & 10324389 & 99.2 & 25.31756 & 0.05284833 & 98.92 & 18.057997 & 0.023136292 & 95.16 & 11.848432 \\
\hline & 0.16103599 & 99.28 & 31.08216 & 0.06101039 & & 19.262946 & 0.025309386 & 95.28 & 12.347252 \\
\hline$A S_{\operatorname{Max}}$ & 0.06374912 & 90.04 & 18.87231 & 0.02607487 & 95.68 & 11.387236 & 0.017109906 & 89.88 & 8.560494 \\
\hline & 0.15006923 & 99.28 & 30.43423 & 0.06685016 & 98.88 & 20.156896 & 0.0250312 & 95.28 & 12.287288 \\
\hline$N_{1}$ & 0.18582309 & 99.32 & 33.38369 & 0.0693412 & 98.88 & 20.485039 & 0.025825856 & 95.4 & 12.462973 \\
\hline$N_{2}$ & 0.18548661 & 99.32 & 33.35509 & 0.06928358 & 98.88 & 20.477026 & 0.025823159 & 95.4 & 12.462382 \\
\hline$N_{3}$ & 0.18097761 & 99.32 & 32.96569 & 0.06826402 & 98.88 & 20.333745 & 0.025761355 & 95.36 & 12.448718 \\
\hline & 0.18515079 & 99.32 & 33.32651 & 0.06922627 & 98.88 & 20.469052 & 0.025820482 & 95.4 & 12.461796 \\
\hline
\end{tabular}


Table A7: Poisson Regression Simulation Results (New Estimators), $\mathrm{P}=4, \mathrm{n}=35$, $\rho=.90$

\begin{tabular}{|c|c|c|c|c|c|c|c|c|c|}
\hline \multirow[b]{2}{*}{$\mathrm{k}$} & \multicolumn{3}{|c|}{ Intercept $=-1$} & \multicolumn{3}{|c|}{ Intercept $=0$} & \multicolumn{3}{|c|}{ Intercept $=1$} \\
\hline & MSE & Perf. & MAPE & MSE & Perf. & MAPE & MSE & Perf. & MAPE \\
\hline$M L E$ & 1.0028166 & 0 & 72.95136 & 0.32629316 & 0 & 43.01376 & 0.11910255 & 0 & 26.0815 \\
\hline$H K$ & 0.6259404 & 95.96 & 58.09197 & 0.22148176 & 96.04 & 35.65563 & 0.106847 & 88.52 & 24.89263 \\
\hline$H K_{2}$ & 0.7530041 & 96.4 & 63.53335 & 0.26191816 & 96.28 & 38.70548 & 0.111842 & 88.56 & 25.38898 \\
\hline$H K B$ & 0.4258919 & 95.16 & 48.38645 & 0.16717313 & 95.72 & 31.16153 & 0.09566814 & 88.08 & 23.69835 \\
\hline$L W$ & 0.9786389 & 96.84 & 72.46727 & 0.31386388 & 96.52 & 42.31657 & 0.1190661 & 88.64 & 26.0788 \\
\hline$H S L$ & 0.3656912 & 94.32 & 43.11879 & 0.13951993 & 95.28 & 28.17122 & 0.07439321 & 85.8 & 20.93152 \\
\hline$A M$ & 0.2267087 & 82.44 & 39.36818 & 0.117466884 & 83.12 & 25.96672 & 0.0713103 & 75.92 & 19.52213 \\
\hline$G M$ & 0.1969764 & 90.32 & 34.20196 & 0.07444337 & 93.16 & 21.0231 & 0.05718861 & 84.12 & 18.60961 \\
\hline Med & 0.2503527 & 93.16 & 37.37616 & 0.11162958 & 94.44 & 25.38812 & 0.07157075 & 86.04 & 20.63378 \\
\hline$K S$ & 0.6336287 & 96 & 58.46267 & 0.2236132 & 96.12 & 35.83325 & 0.10694869 & 88.52 & 24.90179 \\
\hline$K S_{A}$ & 0.4697694 & 95.64 & 50.34719 & 0.17568273 & 95.64 & 31.73933 & 0.0745835 & 85.72 & 20.95392 \\
\hline$K S_{\operatorname{Max}}$ & 0.3162343 & 94.28 & 41.01017 & 0.12127531 & 94.68 & 26.2924 & 0.05811833 & 82.64 & 18.54279 \\
\hline$K S_{\text {Med }}$ & 0.7505575 & 96.6 & 64.51203 & 0.26387883 & 96.16 & 39.04913 & 0.10454921 & 88.4 & 24.61761 \\
\hline$K M_{1}$ & 0.8078521 & 96.6 & 65.96458 & 0.26249854 & 96.2 & 38.82387 & 0.09959921 & 87.92 & 24.04782 \\
\hline$K M_{2}$ & 0.2822983 & 96.08 & 42.31207 & 0.22208673 & 96.28 & 36.70338 & 0.10135592 & 88.52 & 24.40289 \\
\hline$K M_{3}$ & 0.1669752 & 90.4 & 33.07998 & 0.08720549 & 93.44 & 22.80391 & 0.06000056 & 83.32 & 19.00394 \\
\hline$K M_{4}$ & 0.5266961 & 96.6 & 56.58369 & 0.2815697 & 96.44 & 40.64315 & 0.11309634 & 88.64 & 25.5555 \\
\hline$K M_{5}$ & 0.2323945 & 94.16 & 37.23336 & 0.13173783 & 95.6 & 27.89768 & 0.08164897 & 87.32 & 22.05008 \\
\hline$K M_{6}$ & 0.4689782 & 96.52 & 53.57232 & 0.26590633 & 96.4 & 39.6355 & 0.11088386 & 88.64 & 25.34751 \\
\hline$K M_{7}$ & 0.2807417 & 94.92 & 40.75974 & 0.16272259 & 96 & 31.06651 & 0.08912002 & 87.56 & 22.96327 \\
\hline$K M_{8}$ & 0.2796845 & 96.08 & 42.11955 & 0.22112238 & 96.28 & 36.63017 & 0.10129182 & 88.52 & 24.39693 \\
\hline$K M_{9}$ & 0.4911797 & 96 & 53.43322 & 0.22389406 & 96.16 & 408 & 0.10542708 & 88.56 & 24.77087 \\
\hline$K M_{10}$ & 0.4592144 & 96.52 & 53.35941 & 0.27171044 & 96.44 & 40.05113 & 0.11229289 & 88.64 & 25.4 \\
\hline$K M_{11}$ & 0.393491 & 96.08 & 49.06906 & 0.22329482 & 96.2 & 36.45214 & 0.10405479 & 88.56 & 24.64972 \\
\hline$K M_{12}$ & 0.4458503 & 96.52 & 52.40537 & 0.26248275 & 96.4 & 39.4203 & 0.11066108 & 88.64 & 25.328 \\
\hline$K M_{13}$ & 0.5343598 & 96.8 & 57.25454 & 0.29700206 & 96.52 & 41.61702 & 0.11598298 & 88.64 & 25.82724 \\
\hline$K M_{14}$ & 0.4240855 & 96.08 & 50.54584 & 0.22356519 & 96.2 & 36.39067 & 0.1045354 & 88.56 & 24.69246 \\
\hline$G K$ & 0.6132865 & 95.96 & 57.81581 & 0.22133406 & 96.04 & 35.64657 & 0.10683554 & 88.52 & 24.89166 \\
\hline HМO & 0.1412853 & 89.36 & 30.50401 & 0.04986811 & 91.48 & 18.189 & 2033 & 75.2 & 15.20702 \\
\hline$A D_{H M}$ & 0.9889481 & 96.84 & 72.65942 & 0.32560875 & 96.52 & 42.97512 & 0767 & 88.64 & 26.07942 \\
\hline$A D_{M e d}$ & 0.9577302 & 96.76 & 71.73185 & 0.32366889 & 96.52 & 42.86068 & 0.11891765 & 88.64 & 26.06726 \\
\hline$A D_{G M}$ & 0.9389417 & 96.72 & 70.97555 & 0.32060306 & 96.52 & 42.66949 & 88024 & 88.64 & 26.05595 \\
\hline$A D_{A M}$ & 0.7856605 & 95.76 & 65.23363 & 0.29631595 & 96.12 & 40.84617 & 0.11658461 & 88.2 & 25.80934 \\
\hline$Y_{1}$ & 0.3178844 & 95.12 & 43.52736 & 0.20269421 & 95.96 & 34.16569 & 0.10461263 & 88.32 & 24.6274 \\
\hline$Y_{2}$ & 0.5550701 & 96.32 & 56.66075 & 0.27222518 & 96.44 & 39.67159 & 0.11480534 & 88.64 & 25.70346 \\
\hline$Y_{3}$ & 0.4936268 & 96.16 & 53.56132 & 0.2623788 & 96.32 & 39.00832 & 87384 & 88.64 & 25.61925 \\
\hline$Y_{4}$ & 0.2357272 & 93.4 & 38.07677 & 0.15630175 & 95.32 & 29.83 & 1947 & 87.6 & 23.41911 \\
\hline$Y_{5}$ & 0.2792435 & 95.4 & 41.42623 & 0.17039413 & 96.08 & 32.25024 & 0.07743168 & 86.96 & 21.491 \\
\hline$Y_{6}$ & 0.139985 & 89.84 & 31.0727 & 0.06492581 & 94.12 & 19.82318 & 0.0482807 & 80.88 & 17.20025 \\
\hline$Y_{7}$ & 0.147844 & 93.36 & 30.15303 & 0.0945474 & 95.2 & 23.7027 & 0.05924895 & 84.52 & 18.8997 \\
\hline$Y_{8}$ & 0.7496732 & 96.68 & 65.16319 & 0.30064948 & 96.48 & 41.56626 & 0.11668233 & 88.64 & 25.87398 \\
\hline$Y_{9}$ & 0.4587053 & 96.28 & 51.86445 & 0.23190608 & 96.2 & 37.20217 & 0.09614185 & 88.04 & 23.73097 \\
\hline$A H$ & 0.2730753 & 95.8 & 41.03468 & 0.20125881 & 96.2 & 34.84613 & 0.10110996 & 88.56 & 24.35904 \\
\hline$F G$ & 0.1876072 & 94.36 & 34.24967 & 0.12898325 & 95.6 & 28.21911 & 0.07719196 & 87.08 & 21.55358 \\
\hline$A S$ & 0.6181626 & 95.96 & 57.93472 & 0.22140739 & 96.04 & 35.65107 & 0.10684123 & 88.52 & 24.89214 \\
\hline$A S_{\operatorname{Max}}$ & 0.2742711 & 77.12 & 44.57399 & 0.15916801 & 74.92 & 30.58714 & 0.08849338 & 70 & 21.0621 \\
\hline$A S_{\text {Min }}$ & 0.2933738 & 96.36 & 42.81369 & 0.22775428 & 96.36 & 37.2011 & 0.09969512 & 88.44 & 24.23928 \\
\hline$N_{1}$ & 0.9874724 & 96.8 & 72.62615 & 0.32552863 & 96.52 & 42.97059 & 0.11907365 & 88.64 & 26.07918 \\
\hline$N_{2}$ & 0.9351905 & 96.76 & 70.92703 & 0.31745497 & 96.52 & 42.48446 & 0.11855795 & 88.64 & 26.03429 \\
\hline$N_{3}$ & 0.8015774 & 96.44 & 65.83389 & 0.28621763 & 96.36 & 40.45018 & 0.11571181 & 88.64 & 25.77111 \\
\hline$N_{4}$ & 0.8863345 & 96.68 & 69.28141 & 0.30991734 & 96.52 & 42.02044 & 0.11806585 & 88.64 & 25.9911 \\
\hline$C Z_{1}$ & 0.1422857 & 92.24 & 29.8559 & 0.08607488 & 95.32 & 23.07984 & 0.05671529 & 85 & 18.60739 \\
\hline$C Z_{2}$ & 0.293801 & 95.8 & 42.94517 & 0.2026431 & 96.12 & 35.10511 & 0.09581987 & 88.32 & 23.77897 \\
\hline$C Z_{3}$ & 0.2563547 & 95.64 & 40.21304 & 0.18491624 & 96.16 & 33.61944 & 0.08998263 & 87.92 & 23.10407 \\
\hline$C Z_{4}$ & 0.273171 & 95.96 & 41.57966 & 0.20495087 & 96.16 & 35.326 & 0.09649753 & 88.32 & 23.84428 \\
\hline $\mathrm{CZ}_{5}$ & 0.1802911 & 94.36 & 33.62271 & 0.12794375 & 95.6 & 28.10898 & 0.07310121 & 86.72 & 20.98845 \\
\hline
\end{tabular}


Table A8: Poisson Regression Simulation Results (New Estimators), $\mathrm{P}=4, \mathrm{n}=35$, $\rho=.95$

\begin{tabular}{|c|c|c|c|c|c|c|c|c|c|}
\hline \multirow[b]{2}{*}{$\mathrm{k}$} & \multicolumn{3}{|c|}{ Intercept $=-1$} & \multicolumn{3}{|c|}{ Intercept $=0$} & \multicolumn{3}{|c|}{ Intercept $=1$} \\
\hline & MSE & Perf. & MAPE & MSE & Perf. & MAPE & MSE & Perf. & MAPE \\
\hline$M L E$ & 2.0490858 & 0 & 106.03229 & 0.68272769 & 0 & 61.95466 & 0.24061898 & 0 & 37.15363 \\
\hline$H K$ & 1.1725077 & 98.76 & 78.8465 & 0.40728531 & 99.08 & 47.55207 & 0.19736449 & 96.24 & 33.98442 \\
\hline$H K_{2}$ & 1.4319195 & 99.08 & 87.84817 & 0.50489904 & 99.16 & 53.14615 & 0.21482845 & 96.32 & 35.29142 \\
\hline$H K B$ & 0.7290244 & 98.6 & 62.17478 & 0.28168156 & 99.04 & 39.58486 & 0.16484727 & 96.16 & 31.20953 \\
\hline$L W$ & 1.9929189 & 99.12 & 104.85498 & 0.63329823 & 99.12 & 59.90733 & 0.24047855 & 96.36 & 37.14581 \\
\hline$H S L$ & 0.5412752 & 98.52 & 51.72647 & 0.21692201 & 98.8 & 33.33152 & 0.10813219 & 95.08 & 24.61559 \\
\hline$A M$ & 2301093 & 93.48 & 38.58909 & 0.10384248 & 93.12 & 23.92963 & 0.07412403 & 90.32 & 19.68704 \\
\hline$G M$ & 2721952 & 97.36 & 37.763 & 0.09707626 & 97.88 & 22.59061 & .07657127 & 94.88 & 21.01672 \\
\hline Med & 3600331 & 98.24 & 43.08517 & 0.16587689 & 98.52 & 29.86711 & 11001182 & 95.44 & 25.10373 \\
\hline$K S$ & 1.1835254 & 98.8 & 79.3131 & 0.41064721 & 99.08 & 47.78009 & 19761989 & 96.24 & 34.00381 \\
\hline$K S_{A}$ & 0.7907261 & 98.76 & 64.74362 & 0.302241 & 99.04 & 40.4889 & 1844286 & 95.72 & 25.81678 \\
\hline$K S_{\operatorname{Max}}$ & 0.481012 & 98.56 & 49.20137 & 0.19085873 & 98.76 & 31.36301 & .08047272 & 94.48 & 21.00754 \\
\hline$K S_{\text {Med }}$ & 1.4827148 & 99.08 & 91.49611 & 0.52151369 & 99.12 & & 19950655 & 96.32 & 34.10793 \\
\hline$K M_{1}$ & 1.6230686 & 99.08 & 94.867 & 5307369 & 99.12 & 54.88 & 18918462 & 96.28 & 5111 \\
\hline$K M_{2}$ & 0.323035 & 98.88 & 45.8 & 33266813 & 99.16 & 45.8 & 17497648 & 96.28 & 562 \\
\hline$K M_{3}$ & 0.1767835 & 97.4 & 33.1 & 01773 & 97.88 & 23.8 & 02668 & 95.28 & 404 \\
\hline$K M_{4}$ & 0.7600862 & 99.08 & 69.4 & 27297 & 99.12 & 54.6 & 504289 & 96.36 & 016 \\
\hline$K M_{5}$ & 0.3195479 & 98.44 & 42.70 & 9165164 & 98.84 & 33.2 & 203 & 95.92 & 298 \\
\hline$K M_{6}$ & 0.6586645 & 99.08 & 64.672 & 0.44909575 & 99.12 & 52.3946 & & 96.36 & 34.9292 \\
\hline$K M_{7}$ & 0.3875409 & 98.68 & 47.48327 & 0.24732505 & 99.12 & 38.17018 & 14749409 & 96.12 & 29.57832 \\
\hline$K M_{8}$ & 201347 & 98.88 & 45.6 & 33111535 & 99.16 & 45.7 & 81966 & 96.28 & 306 \\
\hline$K M_{9}$ & 5974 & 98.92 & 68.2 & 76738 & 99.12 & 47. & 26238 & 96.28 & 283 \\
\hline$K M_{10}$ & 0.6427024 & 99.08 & & 5158 & 99.12 & 53.502 & & 96.36 & \\
\hline$K M_{11}$ & 0.557195 & 98.92 & 53 & 43427 & 99.16 & 46.82 & & 96.24 & 985 \\
\hline$K M_{12}$ & 0.6209108 & 99.08 & 62.9 & 22939 & 99.12 & 52.03191 & 961 & 96.36 & 8151 \\
\hline$K M_{13}$ & 0.7859176 & 99.12 & 70.79209 & 0.54060519 & 99.12 & 57.08268 & 0.22607365 & 96.36 & 36.31531 \\
\hline$K M_{14}$ & 0.6297965 & 98.92 & 62.0605 & 0.36837659 & 99.16 & 47.00536 & 0.18759267 & 96.28 & 33.35084 \\
\hline$G K$ & 138 & 98.76 & 02 & 2959 & 99.08 & & 762 & 96.24 & 982 \\
\hline HМO & 733 & 97.28 & & 8168 & 98 & & & 93.2 & \\
\hline$A D_{H M}$ & 2.0266048 & 99.12 & 34 & 7217 & 99.12 & & 273 & 96.36 & \\
\hline$A D_{M e d}$ & 1.9582841 & 99.12 & 518 & 4656 & 99. & & & 96.36 & 626 \\
\hline$A D_{G M}$ & 1.9099815 & 99.08 & 102.38225 & 67056636 & 99. & & 70765 & 96.36 & 392 \\
\hline$A D_{A M}$ & 1.587795 & 98.88 & 92.14567 & 0.61877895 & 98.68 & 58.59256 & 0.22988062 & 96.32 & 9579 \\
\hline$Y_{1}$ & 0.4064848 & 98.6 & 48.07854 & 0.31259027 & 99.08 & 42.0 & 0.18520016 & 96.2 & 7972 \\
\hline$Y_{2}$ & 0.8609 & 99 & 70.2 & 07262 & 99.12 & 53. & 11599 & 96.36 & \\
\hline$Y_{3}$ & 0.7065151 & 99.08 & & 7292 & 99.16 & & & 96.36 & \\
\hline$Y_{4}$ & & 97.84 & & 461 & 98. & & & 68 & \\
\hline$Y_{5}$ & & 98.76 & & 980268 & 99.12 & & 42455 & 95.88 & 015 \\
\hline$Y_{6}$ & 1250526 & 96.96 & & 0.05782843 & 98.48 & & & 94.48 & 3896 \\
\hline$Y_{7}$ & 1384759 & 98.16 & 28.90967 & 0.1019143 & 98.72 & 709 & 75584 & 95.24 & 372 \\
\hline$Y_{8}$ & 3635925 & 99.08 & & 5915253 & 99.12 & 824 & 0.23113484 & 96.36 & 2314 \\
\hline$Y_{9}$ & 2624 & 98.92 & & 6758 & 99.1 & & & 96.28 & \\
\hline$A H$ & 0.3250603 & 98.88 & & 38071 & 99.16 & & 69723 & 96.24 & 5836 \\
\hline$F G$ & 0.2309144 & 98.6 & 38.22989 & 0.17617754 & 99.04 & 33.22163 & 1526454 & 96 & 26.65131 \\
\hline$A S$ & 1.1634984 & 98.76 & 78.6432 & 0695493 & 99.08 & & 9734599 & 96.24 & 33.9832 \\
\hline$A S_{\operatorname{Max}}$ & 0.247976 & 90.24 & 41.87416 & 2915501 & 87.84 & 26.97225 & 0.08177367 & 86.96 & 19.89988 \\
\hline$A S_{\text {Min }}$ & 0.3749072 & 98.92 & & & 99. & & & 96.24 & 32.103 \\
\hline$N_{1}$ & 2.0241626 & 99.12 & 105.44458 & 0.68054455 & 99.12 & 61.86692 & 0.24052256 & 96.36 & 37.14771 \\
\hline$N_{2}$ & 1.8990718 & 99.12 & 102.13499 & 0.65769605 & 99.12 & & 0.23871935 & 96.36 & 37.02852 \\
\hline$N_{3}$ & 1.5378549 & 99.04 & 91.58143 & 0.56447416 & 99.12 & 56.36571 & 0.22785017 & 96.36 & 36.26661 \\
\hline$N_{4}$ & 1.7836742 & 99.12 & 98.98846 & 0.63639693 & 99.12 & 59.89399 & 0.23697393 & 96.36 & 36.91192 \\
\hline$C Z_{1}$ & .1353147 & 98.16 & 28.38846 & 0.09294083 & 98.8 & 24.15561 & 0.07055345 & 95.2 & 20.9999 \\
\hline$C Z_{2}$ & 0.3703684 & 98.88 & & 0.30626418 & 99.16 & & 0.16195592 & 96.2 & 31.3205 \\
\hline$C Z_{3}$ & 0.3104124 & 98.8 & & 0.27307893 & & 41.49025 & & 96.16 & 30.02208 \\
\hline$C Z_{4}$ & 0.3238396 & 98.84 & 45.96995 & 0.30784873 & 99.16 & 44.1004 & 0.16411785 & 96.24 & 31.55122 \\
\hline $\mathrm{CZ}_{5}$ & 0.202858 & 98.6 & 35.84632 & 0.17257306 & 99.04 & 32.93484 & 0.1088958 & 95.92 & 25.91458 \\
\hline
\end{tabular}


Table A9: Poisson Regression Simulation Results (New Estimators), $\mathrm{P}=4, \mathrm{n}=35$, $\rho=.99$

\begin{tabular}{|c|c|c|c|c|c|c|c|c|c|}
\hline \multirow[b]{2}{*}{$\mathrm{k}$} & \multicolumn{3}{|c|}{ Intercept $=-1$} & \multicolumn{3}{|c|}{ Intercept $=0$} & \multicolumn{3}{|c|}{ Intercept $=1$} \\
\hline & MSE & Perf. & MAPE & MSE & Perf. & MAPE & MSE & Perf. & MAPE \\
\hline$M L E$ & 11.59862064 & 0 & 248.89533 & 3.92752004 & 0 & 146.02998 & 1.32806587 & 0 & 86.05758 \\
\hline$H K$ & 5.16901858 & 99.96 & 155.40998 & 1.77483503 & 99.96 & 92.17015 & 0.78228575 & 99.96 & 65.89084 \\
\hline$H K_{2}$ & 6.90309291 & 99.96 & 183.30872 & 2.4230536 & 99.96 & 109.98407 & 0.97449011 & 99.96 & 73.40056 \\
\hline$H K B$ & 2.99875138 & 99.96 & 115.39624 & 1.11382591 & 99.96 & 71.03546 & 0.54211062 & 99.96 & 54.38781 \\
\hline$L W$ & 10.43098252 & 100 & 237.88167 & 3.07875993 & 99.96 & 128.44568 & 1.323704 & 99.96 & 85.94975 \\
\hline$H S L$ & 2.05559927 & 99.92 & 87.5429 & 0.76317238 & 99.96 & 52.92151 & 0.2874116 & 99.88 & 34.85554 \\
\hline$A M$ & 0.4015014 & 99.48 & 43.19435 & 0.1900052 & 98.96 & 27.26891 & 0.12001248 & 98.28 & 22.46368 \\
\hline$G M$ & 1.34270517 & 99.72 & 67.17091 & 0.43354699 & 99.92 & 37.67977 & 0.20286711 & 99.84 & 29.57603 \\
\hline Med & 1.48597362 & 99.92 & 76.31322 & 0.59851432 & 99.96 & 48.71072 & 0.31843104 & 99.92 & 38.75027 \\
\hline$K S$ & 5.18756899 & 99.96 & 155.90178 & 1.78197103 & 99.96 & 92.46829 & 0.78369033 & 99.96 & 65.95807 \\
\hline$K S_{A}$ & 2.89638962 & 99.96 & 114.10815 & 1.05639641 & 99.96 & 68.88654 & 0.3871428 & 99.96 & 42.47837 \\
\hline$K S_{\operatorname{Max}}$ & 1.43009884 & 99.92 & 75.91346 & 0.5241464 & 99.96 & 45.45461 & 0.20268776 & 99.88 & 29.22191 \\
\hline$K S_{\text {Med }}$ & 7.62486669 & 100 & 205.82244 & 2.69268656 & 99.96 & 122.47297 & 0.97311624 & 99.96 & 74.53462 \\
\hline$K M_{1}$ & 8.96633329 & 100 & 218.62747 & 2.90633372 & 99.96 & 125.25256 & 0.95625724 & 99.96 & 72.91907 \\
\hline$K M_{2}$ & 0.18411166 & 99.96 & 33.54524 & 0.36497934 & 99.96 & 48.92098 & 0.37004544 & 99.96 & 48.67378 \\
\hline$K M_{3}$ & 0.20857339 & 99.84 & 33.38231 & 0.15239147 & 99.92 & 26.50987 & 0.13609176 & 99.8 & 25.70931 \\
\hline$K M_{4}$ & 0.67791077 & 100 & 64.42767 & 0.89003637 & 99.96 & 76.14187 & 0.68946887 & 99.96 & 65.62485 \\
\hline$K M_{5}$ & 0.6591489 & 99.96 & 57.16986 & 0.42800704 & 100 & 46.20267 & 0.30376206 & 99.96 & 40.77465 \\
\hline$K M_{6}$ & 0.54418465 & 99.96 & 57.51251 & 0.72844981 & 99.96 & 68.8 & 0.60114942 & 99.96 & 61.51946 \\
\hline$K M_{7}$ & 0.75211324 & 99.96 & 62.8852 & 0.53659044 & 99.96 & 53. & 0.38235568 & 99.96 & 46.28176 \\
\hline$K M_{8}$ & 0.18302347 & 99.96 & 33.44503 & 0.36323335 & 99.96 & 48. & 0.36946942 & 99.96 & 48.63503 \\
\hline$K M_{9}$ & 1.81379776 & 99.96 & 100.63576 & 1.05920063 & 99.96 & 76.42052 & 0.63261382 & 99.96 & 60.7316 \\
\hline$K M_{10}$ & 0.53907833 & 99.96 & 57.75614 & 0.795186 & 99.96 & 72.2469 & 0.6576507 & 99.96 & 64.2807 \\
\hline$K M_{11}$ & 0.70688798 & 99.96 & 66.07507 & 0.67655884 & 99.96 & 64.44622 & 0.52076794 & 99.96 & 56.27929 \\
\hline$K M_{12}$ & 0.51352531 & 99.96 & 55.95328 & 0.7129509 & 99.96 & 68.06294 & 0.59611315 & 99.96 & 61.28097 \\
\hline$K M_{13}$ & 0.66209415 & 100 & 61.81 & 1.02232746 & 99.96 & 81.51618 & 0.79199266 & 99.96 & 70.35548 \\
\hline$K M_{14}$ & 0.96815134 & 99.96 & 76.3 & 0.78382182 & 99.96 & 7711 & 0.5568637 & 99.96 & 57.79554 \\
\hline$G K$ & 4.69020908 & 99.96 & 151.83489 & 1.76182077 & 99.96 & 3604 & 0.7816406 & 99.96 & 65.86984 \\
\hline$H M O$ & 0.58235547 & 99.8 & 49.29534 & 0.18748374 & 100 & 27.5 & 0.0805633 & 99.84 & 20.60103 \\
\hline$A D_{H M}$ & 11.41344599 & 100 & 246.80516 & 3.90535099 & 99.96 & 145.59537 & 1.32655119 & 99.96 & 86.01167 \\
\hline$A D_{M e d}$ & 10.94043716 & 100 & 241.48483 & 3.86624192 & 99.96 & 144.79084 & 1.32093833 & 99.96 & 85.82183 \\
\hline$A D_{G M}$ & 10.87683784 & 100 & 238.8368 & 3.82532963 & 99.96 & .753 & 1.31421659 & 99.96 & 85.58268 \\
\hline$A D_{A M}$ & 8.62323277 & 99.96 & 206.55567 & 3.37458248 & 99.92 & 132.0764 & 1.22429409 & 99.92 & 81.71107 \\
\hline$Y_{1}$ & 0.3889054 & 99.92 & 44.1 & 0.47750714 & 99.96 & 49. & 0.47835829 & 99.92 & 50.8935 \\
\hline$Y_{2}$ & 1.63122164 & 99.96 & 90.9 & 1.42371912 & 99.96 & & 0.89584854 & 99.96 & 70.95743 \\
\hline$Y_{3}$ & 0.86121277 & 99.96 & 67.23187 & 0.93968523 & 99.96 & 72.31002 & 0.7626844 & 99.96 & 66.36362 \\
\hline$Y_{4}$ & 0.20732689 & 99.8 & 33.5111 & 0.24735886 & 99.96 & 34.11375 & 0.29407683 & 99.88 & 38.63228 \\
\hline$Y_{5}$ & 0.4747413 & 99.96 & 53.63538 & 0.42385213 & 99.96 & 51.69867 & 0.27387732 & 99.92 & 41.10216 \\
\hline$Y_{6}$ & 0.10175007 & 99.76 & 28.61451 & 0.03075036 & 100 & 12.75715 & 0.03543134 & 99.72 & 13.98985 \\
\hline$Y_{7}$ & 0.08112181 & 99.96 & 23.19876 & 0.06652247 & 99.96 & 18.56974 & 0.07436891 & 99.92 & 20.53821 \\
\hline$Y_{8}$ & 4.87645724 & 100 & 162.47759 & 2.60723129 & 99.96 & 119.33392 & 1.1254286 & 99.96 & 79.51832 \\
\hline$Y_{9}$ & 1.31426707 & 100 & 86.50562 & 0.92196148 & 99.96 & 75.08891 & 0.5303737 & 99.96 & 56.89025 \\
\hline$A H$ & 0.22133229 & 99.96 & 35.7475 & 0.350242 & 99.96 & 46.30618 & 0.3853064 & 99.96 & 49.29951 \\
\hline$F G$ & 0.3175933 & 99.96 & 44.54639 & 0.27209444 & 99.96 & 41.26734 & 0.22002628 & 99.96 & 37.10178 \\
\hline$A S$ & 4.82189324 & 99.96 & 153.30412 & 1.76820306 & 99.96 & 92.05165 & 0.78196254 & 99.96 & 65.88032 \\
\hline$A S_{\operatorname{Max}}$ & 0.17837797 & 98.88 & 35.13783 & 0.10881857 & 97.52 & 22.88834 & 0.09079489 & 96.72 & 19.82571 \\
\hline$A S_{\text {Min }}$ & 0.28871191 & 99.96 & 39.98735 & 0.42808638 & 99.96 & 52.11682 & 0.37345673 & 99.96 & 48.8162 \\
\hline$N_{1}$ & 11.39291187 & 100 & 246.57221 & 3.90280706 & 99.96 & 145.54516 & 1.32637339 & 99.96 & 86.00629 \\
\hline$N_{2}$ & 10.32548969 & 100 & 232.97517 & 3.66809181 & 99.96 & 140.48712 & 1.29595228 & 99.96 & 85.02855 \\
\hline$N_{3}$ & 7.29557829 & 99.96 & 190.65332 & 2.76709845 & 99.96 & 119.23483 & 1.11450628 & 99.96 & 78.74319 \\
\hline$N_{4}$ & 9.41463899 & 100 & 221.119 & 3.46491363 & 99.96 & 135.99109 & 1.26739478 & 99.96 & 84.09359 \\
\hline$C Z_{1}$ & 0.10197737 & 99.96 & 23.74936 & 0.08447563 & 99.96 & 20.46401 & 0.08003285 & 99.84 & 21.65286 \\
\hline$C Z_{2}$ & 0.3227991 & 99.96 & 45.22677 & 0.42804446 & 99.96 & 52.87905 & 0.3676123 & 99.96 & 48.35791 \\
\hline$C Z_{3}$ & 0.22957846 & 99.96 & 37.82974 & 0.35245554 & 99.96 & 47.9687 & 0.31684246 & 99.96 & 44.99306 \\
\hline$C Z_{4}$ & 0.24479372 & 99.96 & 39.17488 & 0.37064755 & 99.96 & 49.35962 & 0.34924029 & 99.96 & 47.46317 \\
\hline$C Z_{5}$ & 0.13577832 & 99.96 & 28.88463 & 0.20803069 & 99.96 & 36.1392 & 0.20001428 & 99.92 & 35.46409 \\
\hline
\end{tabular}


Table A10: Poisson Regression Simulation Results (New Estimators), $\mathrm{P}=4, \mathrm{n}=600$, $\rho=.90$

\begin{tabular}{|c|c|c|c|c|c|c|c|c|c|}
\hline \multirow[b]{2}{*}{$\mathrm{k}$} & \multicolumn{3}{|c|}{ Intercept $=-1$} & \multicolumn{3}{|c|}{ Intercept $=0$} & \multicolumn{3}{|c|}{ Intercept $=1$} \\
\hline & MSE & Perf. & MAPE & MSE & Perf. & MAPE & MSE & Perf. & MAPE \\
\hline$M L E$ & 0.01620663 & 0 & 10.073359 & 0.006043302 & 0 & 6.132135 & 0.002303969 & 0 & 3.795928 \\
\hline$H K$ & 01596561 & 69.64 & 9.999736 & .005982533 & 58.08 & 6.103113 & 0.002300022 & 54.24 & 3.793071 \\
\hline$H K_{2}$ & 01608755 & 69.88 & 10.037473 & 0.006012503 & 58.24 & 6.117298 & 0.002301814 & 54.28 & 3.794349 \\
\hline$H K B$ & 01575442 & 68.96 & 9.937435 & 0.005925712 & 57.52 & 6.075488 & 0.002295519 & 54.24 & 3.789747 \\
\hline$L W$ & 0162066 & 70.24 & 10.073349 & 0.006043144 & 58.4 & 6.132059 & 0.002303969 & 54.36 & 3.795928 \\
\hline$H S L$ & 01545002 & 67.88 & 9.854192 & 0.00585651 & 56.32 & 6.039524 & 0.002277344 & 53.32 & 3.776254 \\
\hline$A M$ & 3763598 & 47.2 & 13.610251 & 0.014407748 & 42.88 & & 8309309 & 41.84 & 5056 \\
\hline$G M$ & 01510674 & 62.2 & 9.762749 & 0.005698167 & 52.6 & 5.974297 & 0.002299301 & 51.4 & 3.783184 \\
\hline Med & 01530615 & 66.72 & 9.808704 & .005743778 & 55.96 & 5.985457 & 0.002290523 & 52.76 & 3.785477 \\
\hline$K S$ & 0.01597037 & 69.64 & 10.001204 & 0.005983433 & 58.08 & 6.103533 & 0.002300033 & 54.24 & 3.793079 \\
\hline$K S_{A}$ & 01579574 & 69.08 & 9.950039 & 0.005916562 & 57.2 & 6.066 & 0.002273679 & 52.84 & 3.769709 \\
\hline$K S_{\operatorname{Max}}$ & 01545169 & 66.72 & 9.867583 & 591033 & 54.4 & 6.078 & 02433825 & 48.6 & 3.870748 \\
\hline$K S_{\text {Med }}$ & 0.01610357 & 69.92 & 10.0 & 11022 & 58.24 & & 2296337 & & 177 \\
\hline$K M_{1}$ & 0.0160963 & 69.88 & 10.03 & 000161 & 58.16 & & 02291947 & 54.12 & 3.786961 \\
\hline$K M_{2}$ & 0.01595115 & 69.64 & 9.997134 & 018864 & 58.24 & 6.120 & 02299218 & 54.24 & 3.792453 \\
\hline$K M_{3}$ & 0.01637027 & 61.36 & 10.116814 & 980907 & 54.04 & 10477 & 0.002431234 & 51.08 & 3.834475 \\
\hline$K M_{4}$ & 01611974 & 70 & 10.047074 & .006035602 & 58.32 & 6.128491 & 0.002302555 & 54.28 & 3.794914 \\
\hline$K M_{5}$ & 01545909 & 67.92 & 9.84 & 59478 & 57.2 & 6.05 & 0.002287243 & 54 & \\
\hline$K M_{6}$ & 07603 & 69.92 & 10.0 & 2015 & & & 230208 & & \\
\hline$K M_{7}$ & 1570461 & 68.8 & & & 57. & & 292742 & & \\
\hline$K M_{8}$ & 1594879 & 69.64 & & 8698 & & & 2299212 & & 2449 \\
\hline$K M_{9}$ & 01596636 & 69.72 & 10.000 & 99479 & 58.1 & & 2299844 & 54.24 & 3.792927 \\
\hline$K M_{10}$ & 1610224 & 69.96 & 10.04 & 034522 & 58.32 & 6.127 & 0.002302457 & 54.28 & 4839 \\
\hline$K M_{11}$ & 01596134 & 69.72 & & 008281 & 58. & 531 & 00229965 & 54.24 & 2777 \\
\hline$K M_{12}$ & 1607092 & 69.92 & 10.032501 & 603163 & 58.2 & 566 & 2302063 & 54 & 548 \\
\hline$K M_{13}$ & 6162 & 70.0 & & 0849 & & & 303409 & & \\
\hline$K M_{14}$ & 96321 & 69.72 & & & & & & & \\
\hline$G K$ & 96559 & 69.64 & & 2532 & 58.08 & & 2300022 & & 071 \\
\hline$H M O$ & 27399 & 6.44 & 18.6 & 9008 & 3.4 & 72 & & & 9.26 \\
\hline$A D_{H M}$ & 162066 & 70.24 & 10.073349 & 043299 & 58.4 & 6.132133 & 0.002303969 & 54.36 & 5928 \\
\hline$A D_{M e d}$ & 1620653 & 70.24 & 10.07 & 43292 & 58. & & 2303968 & & 928 \\
\hline$A D_{G M}$ & 0638 & 70.24 & 10.0 & 278 & 58. & 6.1 & 303967 & & \\
\hline$A D_{A M}$ & & 70.08 & 10. & 489 & 58. & & 241 & & \\
\hline$Y_{1}$ & 9838 & 69.88 & & & 58.2 & & & & \\
\hline$Y_{2}$ & 9025 & 70.2 & & & & & & & \\
\hline$Y_{3}$ & 1617591 & 70.08 & 10.06 & 39315 & & & 303773 & & 5783 \\
\hline$Y_{4}$ & 01602469 & 69.16 & 10.023441 & 20957 & 58.0 & 6.120243 & 0.002301386 & 54.16 & 3.793901 \\
\hline$Y_{5}$ & 1456897 & 65.32 & & 60909 & 56.56 & 512 & 0.002231423 & 50 & 3.744394 \\
\hline$Y_{6}$ & 7597 & 30.44 & 12.42 & 2493 & 37. & & 282635 & 36.16 & 734 \\
\hline$Y_{7}$ & & 52.8 & & & 49.68 & & 392 & & 227 \\
\hline$Y_{8}$ & & 70.2 & 10.0 & & & & 382 & & 864 \\
\hline$Y_{9}$ & 0152334 & 68 & & 6743 & 57.16 & & 256839 & 52.76 & 1816 \\
\hline$A H$ & 1584167 & 69.4 & & 00852 & 58.2 & & 2298833 & 54. & 3.792202 \\
\hline$F G$ & 518805 & 67.6 & & 97268 & & & 2283981 & 53 & \\
\hline$A S$ & 9656 & 69.64 & & 2532 & $58 .($ & & 300022 & & 071 \\
\hline$A S_{\text {Max }}$ & & 34.68 & 16.8 & 7226 & & & 090732 & 35.52 & 3172 \\
\hline$A S_{\text {Min }}$ & 0159408 & 69.64 & & 0.006024652 & 58.28 & 6.123116 & 0.002298759 & 54.24 & 3.792112 \\
\hline$N_{1}$ & 0.0162066 & 70.24 & 10.073348 & 0.006043299 & 58.4 & 6.132133 & 0.002303969 & 54.36 & 3.795928 \\
\hline$N_{2}$ & 0.01620388 & 70.24 & 07254 & 942869 & 58.4 & 1927 & 0.00230395 & 54.36 & 3.795914 \\
\hline$N_{3}$ & 1617317 & 70.08 & 10.0 & 036557 & 58.28 & 891 & 0.0023036 & 54.32 & 3.795658 \\
\hline$N_{4}$ & 0.01620122 & 70.2 & 10.071748 & 0.006042451 & & 6.131727 & 0.002303932 & 54.36 & 3.795901 \\
\hline$C Z_{1}$ & 0.014144 & 63.84 & & 0.005754112 & 56.24 & 5.991919 & 0.0022547 & 52.44 & 3.760182 \\
\hline$C Z_{2}$ & 0.01568753 & 68.96 & 9.917033 & 0.005977624 & 58.12 & 6.100708 & 0.002291577 & 54.08 & 3.786899 \\
\hline$C Z_{3}$ & 0.01538705 & 68.16 & 9.82522 & 0.005922708 & 57.64 & 6.074603 & 0.002272918 & 53.32 & 3.773588 \\
\hline$C Z_{4}$ & 0.01560638 & 68.84 & 9.891297 & 0.005959494 & 57.84 & 6.092162 & 0.002291772 & 54.08 & 3.787008 \\
\hline$C Z_{5}$ & 0.01456474 & 65.32 & 9.570222 & 0.005757852 & 56.56 & 5.993975 & 0.002231415 & 50.2 & 3.744385 \\
\hline
\end{tabular}


Table A11: Poisson Regression Simulation Results (New Estimators), $\mathrm{P}=4, \mathrm{n}=600$, $\rho=.95$

\begin{tabular}{|c|c|c|c|c|c|c|c|c|c|}
\hline \multirow[b]{2}{*}{$\mathrm{k}$} & \multicolumn{3}{|c|}{ Intercept $=-1$} & \multicolumn{3}{|c|}{ Intercept $=0$} & \multicolumn{3}{|c|}{ Intercept $=1$} \\
\hline & MSE & Perf. & MAPE & MSE & Perf. & MAPE & MSE & Perf. & MAPE \\
\hline$M L E$ & 0.03283609 & 0 & 14.30179 & 0.012641723 & 0 & 8.784325 & 0.004458053 & 0 & 5.219407 \\
\hline$H K$ & 0.03178934 & 83.04 & 14.0862 & 0.012375353 & 72.08 & 8.695585 & 0.004443436 & 59.32 & 5.211708 \\
\hline$H K_{2}$ & 0323332 & 83.28 & 14.20044 & 0.012513937 & 72.2 & 8.741851 & 0.004449535 & 59.36 & 5.21495 \\
\hline$H K B$ & 0.0309322 & 82.96 & 13.9125 & 0.012153615 & 71.52 & 8.621232 & 0.004424512 & 59.24 & 5.201768 \\
\hline$L W$ & 0.03283595 & 83.36 & 14.30177 & 0.012640724 & 72.48 & 8.784002 & 0.004458053 & 59.4 & 5.219407 \\
\hline$H S L$ & 0.03008376 & 82.36 & 13.74228 & 011878693 & 70.8 & 8.520262 & 004311223 & 58.24 & 5.138742 \\
\hline$A M$ & 04255273 & 65.52 & 15.30739 & 15959753 & 61.88 & & 156198 & 48.92 & 5.747974 \\
\hline$G M$ & 02577061 & 78.96 & 12.77741 & 10731835 & 67.8 & 8.14 & 426156 & 56.88 & 5.113322 \\
\hline Med & 0.0291073 & 82.16 & 13.54314 & 1620123 & 70.36 & 3405 & 327712 & 58.32 & 8027 \\
\hline$K S$ & 0.03180976 & 83.04 & 14.09046 & 012379305 & 72.08 & 8.696897 & 443471 & 59.32 & 5.211726 \\
\hline$K S_{A}$ & 03164172 & 83 & 14.06504 & 12258344 & 71.76 & 8.650547 & 0433573 & 57.84 & 5.15112 \\
\hline$K S_{\text {Max }}$ & 0.02997134 & 82.08 & 13.71779 & 0.011808755 & 70.52 & 8.498511 & 0.004369716 & 54.84 & 5.15665 \\
\hline$K S_{\text {Med }}$ & 0.03254171 & 83.32 & 14.24221 & 012549976 & 72.24 & 8.753561 & 0.004436427 & 59.24 & 5.207772 \\
\hline$K M_{1}$ & 55968 & 83.36 & 14.24624 & 532299 & 72.16 & 8.7 & 24548 & 59.2 & 423 \\
\hline$K M_{2}$ & 0.03177804 & 83.04 & 14.09055 & 0.012544726 & 72.28 & 8.75237 & 443888 & 59.28 & 5.20 \\
\hline$K M_{3}$ & 0.02730549 & 77.92 & 13.1245 & 0.011461416 & 69 & 8.388831 & 332923 & 56.76 & 1523 \\
\hline$K M_{4}$ & 0.03245824 & 83.36 & 14.22646 & 2608234 & 72.44 & 222 & 52896 & 59.4 & 709 \\
\hline$K M_{5}$ & 0.02972028 & 82.4 & 13.65365 & 012071814 & 71.36 & 8.59626 & 390743 & 59.04 & 5.184039 \\
\hline$K M_{6}$ & 03225032 & 83.24 & 14.18237 & 012592082 & 72.44 & 8.768044 & 51287 & 59.4 & 5.215872 \\
\hline$K M_{7}$ & 03085605 & 82.88 & 13.9054 & 012264699 & 71.68 & 274 & 408135 & 59.12 & 5.192882 \\
\hline$K M_{8}$ & 76835 & 83.04 & 14.0 & 44041 & 72.24 & & 38859 & 59.28 & 408 \\
\hline$K M_{9}$ & 0.03180853 & 83.04 & 14.0 & 5156 & 72.16 & & 42279 & 59.28 & \\
\hline$K M_{10}$ & 0.03238796 & 83.36 & 14.21 & 60411 & 72.44 & & 152535 & 59.4 & 525 \\
\hline$K M_{11}$ & .03179953 & 83.04 & 14.09 & 96219 & 72.2 & & 177 & 59.28 & \\
\hline$K M_{12}$ & 0.032231 & 83.24 & 14.1 & 90679 & 72.44 & & 1218 & 59.4 & 5.215837 \\
\hline$K M_{13}$ & 0.03263812 & 83.36 & 14.262 & 012630881 & 72.44 & 8.780751 & 456176 & 59.4 & 5.218426 \\
\hline$K M_{14}$ & 0.03180332 & 83.04 & 14.0924 & 83768 & 72.16 & & 41566 & 59.28 & 0772 \\
\hline$G K$ & 928 & 83.04 & 14.0 & & 72.08 & & & 59.32 & \\
\hline$H M O$ & 9073 & 43. & 14.7 & & 36.12 & & & 6.04 & \\
\hline$A D_{H M}$ & 33595 & 83.36 & 14.3 & 1711 & 72.48 & & 8053 & 59.4 & 5.219407 \\
\hline$A D_{M e d}$ & 03283577 & 83.36 & 14.30 & 41693 & 72.48 & & 58052 & 59.4 & 5.21 \\
\hline$A D_{G M}$ & 0.03283501 & 83.36 & 14.3 & 4165 & 72.48 & & 45805 & 59.4 & 5.219405 \\
\hline$A D_{A M}$ & 0.03294206 & 83.16 & 14.31444 & 32522 & 72.4 & 8715 & 67534 & 59.32 & 5.22056 \\
\hline$Y_{1}$ & 0.03221598 & 83.12 & 14.17024 & 85463 & 72.32 & 7816 & 54799 & 59.4 & $5.21^{\prime}$ \\
\hline$Y_{2}$ & & 83. & 14.2 & & 72.44 & & & 9.4 & \\
\hline$Y_{3}$ & 6553 & 83.36 & 14.2 & & 72.44 & & 105 & 9.4 & \\
\hline$Y_{4}$ & 6408 & 82.68 & 14.0 & 7701 & 72.12 & & 50864 & 59.36 & 5.21 \\
\hline$Y_{5}$ & 30253 & 81.32 & 13.12718 & 5675 & 70.68 & 146 & 17271 & 56.96 & 5.063284 \\
\hline$Y_{6}$ & 02395025 & 56.72 & 12.74712 & 5967 & 56.5 & & 10006 & 43.12 & 5.190265 \\
\hline$Y_{7}$ & 01973611 & 73 & 11.23881 & 90266 & 65.36 & 6083 & 67121 & 52.44 & 4.933192 \\
\hline$Y_{8}$ & 80328 & 83.36 & 14.29533 & 38151 & 72.44 & & 7683 & 59.4 & 5.214 \\
\hline$Y_{9}$ & 02958007 & 82.52 & & & 71.44 & & 22631 & 58.28 & 5.146124 \\
\hline$A H$ & 0.03129342 & 83 & 13.98778 & 1247223 & 72.2 & & 43834 & 59.28 & 5.209042 \\
\hline$F G$ & 0.02864674 & 82.2 & 13.42772 & 45024 & 71.24 & 8.585597 & 378797 & 58.8 & 5.177546 \\
\hline$A S$ & 03178931 & 83.04 & 14.08619 & 375352 & 72.08 & 8.695585 & 143436 & 59.32 & 5.211708 \\
\hline & .05783985 & 55.36 & 17.5 & 77327 & 55.44 & & 011483 & 41.64 & 6.421425 \\
\hline$A S_{\text {Min }}$ & 0.03175868 & 83.08 & 14.08869 & .012570698 & 72.44 & 8.760995 & 436557 & 59.28 & 5.208242 \\
\hline$N_{1}$ & 0.03283594 & 83.36 & 14.30176 & & 72.48 & & 58053 & 59.4 & 5.219407 \\
\hline$N_{2}$ & 0.03282464 & 83.36 & 14.29951 & 0.012639954 & 72.48 & 8.783747 & 0.004457982 & 59.4 & 5.21937 \\
\hline$N_{3}$ & 0.0326862 & 83.36 & 14.27176 & 0.012612044 & 72.44 & 8.774537 & 0.004456521 & 59.4 & 5.218614 \\
\hline$N_{4}$ & 0.03281344 & 83.36 & 14.29728 & 0.012638221 & 72.44 & 8.783181 & 0.004457913 & 59.4 & 5.219335 \\
\hline$C Z_{1}$ & 02437126 & 80.16 & & .011500744 & 70.32 & 00618 & 04256092 & 57.92 & 5.111398 \\
\hline$C Z_{2}$ & 0.03083215 & 82.96 & 13.89413 & & 71.96 & & 0.004412646 & 59.12 & 5.195458 \\
\hline$C Z_{3}$ & 0.02989816 & 82.68 & 13.69615 & 0.012214943 & 71.68 & 8.641694 & 0.004355329 & 58.68 & 5.164699 \\
\hline$C Z_{4}$ & 0.03051094 & 82.84 & 13.82631 & 0.012316673 & 71.8 & 8.675677 & 0.004410763 & 59.08 & 5.194413 \\
\hline$C Z_{5}$ & 0.02720618 & 81.28 & 13.10821 & 0.011667485 & 70.68 & 8.456167 & 0.004172455 & 56.96 & 5.063144 \\
\hline
\end{tabular}


Table A12: Poisson Regression Simulation Results (New Estimators), $\mathrm{P}=4, \mathrm{n}=600$, $\rho=.99$

\begin{tabular}{|c|c|c|c|c|c|c|c|c|c|}
\hline \multirow[b]{2}{*}{$\mathrm{k}$} & \multicolumn{3}{|c|}{ Intercept $=-1$} & \multicolumn{3}{|c|}{ Intercept $=0$} & \multicolumn{3}{|c|}{ Intercept $=1$} \\
\hline & MSE & Perf. & MAPE & MSE & Perf. & MAPE & MSE & Perf. & MAPE \\
\hline$M L E$ & .18575322 & 0 & 33.39513 & .068427073 & 0 & 20.262242 & .025228958 & 0 & 12.365021 \\
\hline$H K$ & 16108059 & 99.8 & 31.13023 & .060303723 & 99.16 & 9.066425 & 0.024718464 & 5.72 & 12.249292 \\
\hline$H K_{2}$ & 17201607 & 99.84 & 32.20104 & .064587992 & 99.16 & 9.715722 & .02492239 & 5.72 & \\
\hline$H K B$ & 14037237 & 99.8 & 29.23153 & .055243693 & 99.12 & 18.301745 & 0.02404708 & 95.6 & 12.093563 \\
\hline$L W$ & 18574814 & 99.8 & 33.39472 & .068361936 & 99.2 & 20.253626 & 0.025228946 & 95.72 & 12.365018 \\
\hline$H S L$ & 2407945 & 99.8 & 27.63612 & .051362889 & 99.08 & 17.754366 & 0.019241924 & 95.32 & \\
\hline$A M$ & 35484 & 93.28 & & 467213 & 97.2 & & & & \\
\hline$G M$ & 87112 & 99.32 & 20.6 & 73179 & 99 & & 7995052 & 5.16 & \\
\hline Med & 268139 & 99.76 & 25.4 & & 99.04 & & 55026 & 5.28 & \\
\hline$K S$ & 45497 & 99.8 & 31.1 & 14998 & 99.16 & & 719661 & 5.72 & \\
\hline$K S_{A}$ & 5925927 & 99.84 & 31.1 & 32577 & 99.16 & & & 5.52 & 11.738 \\
\hline$K S_{\text {Max }}$ & 12512679 & 99.8 & 27.7 & 507124 & 99.08 & 17.78 & 517296 & 95.28 & \\
\hline$K S_{\text {Med }}$ & 3368755 & 99.8 & 33.2 & 598052 & 99.2 & & & 5.72 & 12.31 \\
\hline & 9005 & 99.8 & 57 & & 99.2 & & & 5.72 & \\
\hline$K M_{2}$ & 84199 & 99.84 & & 80835 & 99.16 & & 53063 & 95.72 & 12.2 \\
\hline$K M_{3}$ & 7944184 & 98.88 & 21.6 & 86032 & 99.08 & & 08759 & 95 & \\
\hline & 40776 & 99.84 & & & 99.2 & & 713 & 95.72 & \\
\hline$K M_{5}$ & 4098 & 99.8 & & & & & & & \\
\hline$K M_{6}$ & 96816 & 99.84 & & 37125 & 99.2 & & 992092 & 95.72 & \\
\hline$K M_{7}$ & 3515 & 99.8 & & 934 & 99. & & & ס & \\
\hline & & 99.84 & & & 99 & & & 72 & \\
\hline$K M_{9}$ & & 99.8 & & & 99.1 & & & 95 & \\
\hline$K M_{10}$ & & 99.84 & & & & & & & \\
\hline$K M_{11}$ & & 99.8 & & & 99.16 & & & & \\
\hline$K M_{12}$ & 8726 & 99.84 & & & 99.2 & & & & \\
\hline$K M_{13}$ & 4017 & 99.84 & & 4776 & 99. & & & 95. & \\
\hline & & 99.8 & & & 99. & & & 2 & \\
\hline$G K$ & & & & & & & & & \\
\hline$H M O$ & & 97.8 & & & & & & & \\
\hline$A D_{H M}$ & & & & & & & & & \\
\hline & & & & & & & & & \\
\hline$A D_{G M}$ & & & & & & & & & \\
\hline & & 99.76 & & & & & & 95 & 12. \\
\hline$Y_{1}$ & & & & & & & & & \\
\hline$Y_{2}$ & & & & & & & & & \\
\hline$Y_{3}$ & & 99.8 & & & & & & & \\
\hline$Y_{4}$ & & & & & & & & & \\
\hline$Y_{5}$ & & 99.72 & & & & & & & \\
\hline$Y_{6}$ & & & & & & & & & \\
\hline$Y_{7}$ & & 98.56 & & 6749 & 98. & & & & \\
\hline$Y_{8}$ & & & & & & & & & \\
\hline$Y_{9}$ & & 99.72 & & & & & & & \\
\hline$A H$ & & & & & & & & & \\
\hline$F G$ & & 99.68 & & & & & & & \\
\hline & & & & & & & & & \\
\hline & & & & & & & & & \\
\hline & & & & & & & & & \\
\hline$N_{1}$ & & & & & & & & & \\
\hline$N_{2}$ & & & & & & & & & \\
\hline$N_{3}$ & & 99.84 & & & 99.2 & & & & 12.350717 \\
\hline$N_{4}$ & 507373 & 99.8 & & 3547 & 99.2 & 20.246 & 5223707 & 95.72 & 12.363848 \\
\hline$C Z_{1}$ & & 99.64 & & & 99.12 & & 0.019479372 & & 10.940886 \\
\hline & & & & & & & & & \\
\hline$C_{3}$ & & & & & & & & & \\
\hline$C Z_{4}$ & & 99.76 & & & 99.16 & & 0.023927464 & 95.56 & 12.06258 \\
\hline$C Z_{5}$ & 0.09949787 & 99.68 & 24.99212 & 0.050677502 & 99.12 & 17.621805 & 0.019444271 & 95.24 & 10.941036 \\
\hline
\end{tabular}

